\title{
Healthcare workers' behaviours and personal determinants affecting sexual and reproductive healthcare service provision to adolescents in South Africa
}

Citation for published version (APA):

Jonas, N. K. (2017). Healthcare workers' behaviours and personal determinants affecting sexual and reproductive healthcare service provision to adolescents in South Africa. [Doctoral Thesis, Maastricht University]. Maastricht University. https://doi.org/10.26481/dis.20170928nkj

Document status and date:

Published: 01/01/2017

DOI:

10.26481/dis.20170928nkj

Document Version:

Publisher's PDF, also known as Version of record

Please check the document version of this publication:

- A submitted manuscript is the version of the article upon submission and before peer-review. There can be important differences between the submitted version and the official published version of record.

People interested in the research are advised to contact the author for the final version of the publication, or visit the DOI to the publisher's website.

- The final author version and the galley proof are versions of the publication after peer review.

- The final published version features the final layout of the paper including the volume, issue and page numbers.

Link to publication

\footnotetext{
General rights rights.

- You may freely distribute the URL identifying the publication in the public portal. please follow below link for the End User Agreement:

www.umlib.nl/taverne-license

Take down policy

If you believe that this document breaches copyright please contact us at:

repository@maastrichtuniversity.nl

providing details and we will investigate your claim.
}

Copyright and moral rights for the publications made accessible in the public portal are retained by the authors and/or other copyright owners and it is a condition of accessing publications that users recognise and abide by the legal requirements associated with these

- Users may download and print one copy of any publication from the public portal for the purpose of private study or research.

- You may not further distribute the material or use it for any profit-making activity or commercial gain

If the publication is distributed under the terms of Article $25 \mathrm{fa}$ of the Dutch Copyright Act, indicated by the "Taverne" license above, 


\title{
Healthcare Workers' Behaviours and Personal Determinants Affecting Sexual and Reproductive Healthcare Service Provision to Adolescents in South Africa
}

\author{
DISSERTATION \\ to obtain the degree of Doctor at Maastricht University, \\ on the authority of the Rector Magnificus, Prof. Dr. Rianne M. Letschert \\ in accordance with the decision of the Board of Deans, \\ to be defended in public on
}

Thursday, 28 ${ }^{\text {th }}$ September 2017, at 12:00 hours

by

NCEBAKAZI KIM JONAS 


\section{PROMOTOR}

Prof. dr. H.W. van den Borne

\section{CO-PROMOTORS}

Dr. Rik Crutzen

Prof. dr. S.P. Reddy (Human Science Research Council, University of the Western Cape)

\section{ASSESSMENT COMMITTEE}

Prof. dr. N.K. de Vries

Dr. J. Alaii, (USAID/ Uganda)

Dr. C. Hoving

Prof. dr. G.J. Kok

Prof. dr. L. Lechner (Open University Heerlen)

The research presented in this dissertation was conducted at the School for Public Health and Primary Care: CAPHRI, Department of Health Promotion, of the Maastricht University. CAPHRI participates in the research School Care, Netherlands School of Primary Care Research.

The research presented in this dissertation was supported by the Dutch Ministry for Foreign Affairs (through Nuffic) and the National Research Foundation (NRF) of South Africa. Opinion expressed and conclusions arrived at, are those of the authors and are not necessarily to be attributed to the NRF. 


\section{Contents}

CHAPTER 1............................................................................................................................................................... 4

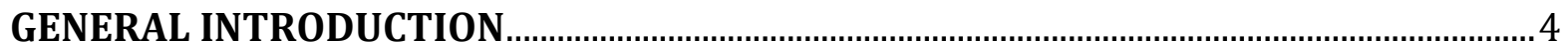

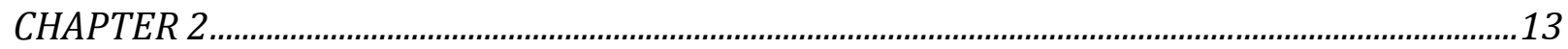

Jonas K, Crutzen R, van den Borne B, Sewpaul R, Reddy P. Teenage pregnancy rates and associations with other health risk behaviours: a three-wave cross-sectional study among

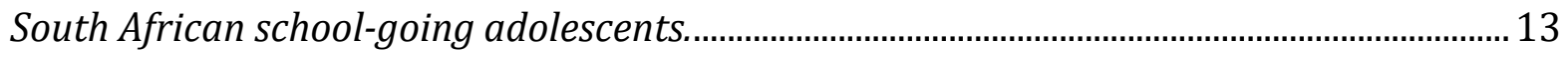

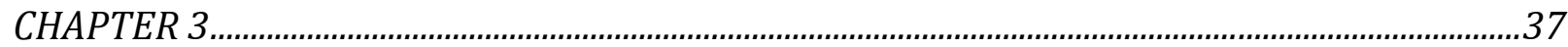

Jonas K, Crutzen R, van den Borne B, Reddy P. Healthcare workers' behaviors and personal determinants associated with providing adequate sexual and reproductive healthcare services in sub-Saharan Africa: a systematic review. 37

CHAPTER 4 72

Jonas K, Reddy P, van den Borne B, Sewpaul R, Nyembezi A, Naidoo P, Crutzen R. Predictors of nurses' and midwives' intentions to provide maternal and child healthcare services to adolescents in South Africa 72 CHAPTER 5. 90

Jonas K, Crutzen R, Krumeich A, Roman N, van den Borne B, and Reddy P. Healthcare workers beliefs, motivation and behaviours affecting adequate provision of sexual and reproductive healthcare services to adolescents in Cape Town, South Africa. A qualitative study. 90

CHAPTER 6. 116

Jonas K, Roman N, Reddy P, Krumeich A, van den Borne B, and Crutzen R. Healthcare workers' perceptions of adolescents accessing and utilizing sexual and reproductive healthcare services in Cape Town, South Africa. ........................................................... 116

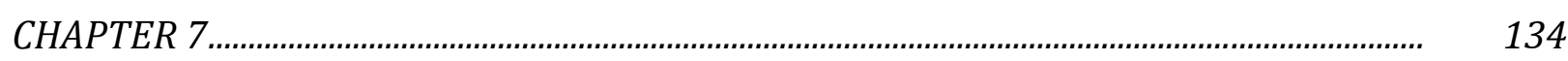

GENERAL DISCUSSION ............................................................................................ 134

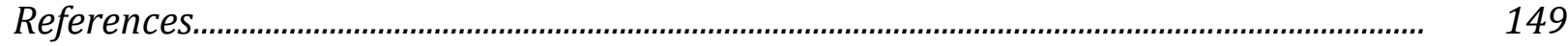

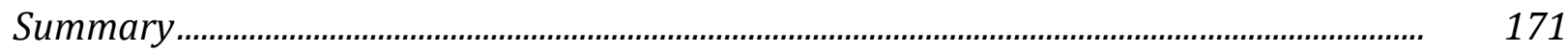

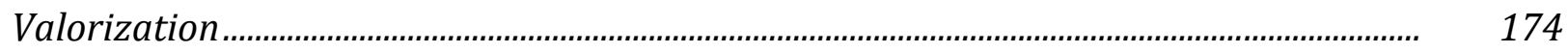

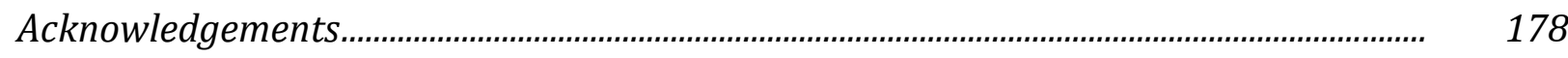

Author resume

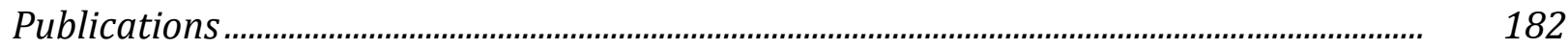




\section{GENERAL INTRODUCTION}

\section{CHAPTER 1}

\section{Sexual and Reproductive Healthcare services in the global context}

According to the International Conference on Population and Development (ICPD) and the WHO, reproductive health is defined as "a state of complete physical, mental and social well-being and not merely the absence of disease or infirmity, in all matters relating to the reproductive system and to its functions and processes" [WHO, 2015; Cohen et al, 1994]. Thus, reproductive health implies that everyone is able to have a satisfying and safe sex life and that they have the capability to reproduce and the freedom to decide if, when and how often to do so [WHO, 2015]. Inherent to this definition are the rights of men and women, including adolescents, to be well-informed and to have complete access to safe, effective, affordable and acceptable methods of contraception of their own choice. Additionally, all women, including adolescent girls who happen to fall pregnant have the right to access appropriate healthcare services that will enable them to go safely through their pregnancy and childbirth and that everyone is provided with the best chance of having a healthy infant [WHO, 2015].

The ICPD Program of Action describes a wide range of services covered under the umbrella of sexual and reproductive healthcare (SRH) services, such as family-planning (FP) counseling and services; prenatal and postnatal care and delivery; termination of pregnancy (TOP) or abortion services and post-abortion care (PAC); treatment and prevention of reproductive tract and sexually transmitted infections (STIs), including human immunodeficiency virus (HIV); and information and counseling on human sexuality [Cohen et al,, 1994]. Therefore, this means that complete access to, and availability of SRH services go hand-in-hand in order for everyone, especially adolescents to fulfill their SRH needs.

Adolescents have the right to fully access and utilize SRH services for their needs as emphasized and supported by the United Nations Population Fund Agency (UNPFA) and the World Health Organization (WHO) [WHO, 2014; UNPFA, 2013]. The availability and use of these services are essential to improve adolescents' SRH outcomes [Hindin et al., 2009; Singh et al., 2005]. However, adolescents under-utilize SRH services, particularly in low- and middle- income countries (LMICs), including South Africa (SA) [WHO, 2014; 
UNPFA, 2013; Mngadi et al., 2008; Warenius et al., 2012; Hindin et al., 2009; Singh et al., 2005]. This under-utilization undermines the global efforts to prioritize adolescents' access to and utilization of SRH services, and is a contributing factor to the high rates of teenage pregnancy and unsafe abortions in SA [Reddy et al., 2011; Macleod et al., 2010].

\section{Public health policy regarding sexual and reproductive health in South Africa}

SA, as recognized globally by its impressive and liberal policies and guidelines regarding human rights and healthcare services, has indeed made great progress since the end of apartheid in 1994. However, there are still numerous challenges in adequately providing primary healthcare services, such as SRH services, especially to young people. According to Ramkissoon et al (2010), implementation of and complete adherence to the guidelines and policies, and healthcare workers' performance are some of the challenges hindering adequate provision of the primary healthcare services, including SRH services like FP services.

SA particularly stands out for its comprehensive formal rights framework where reproductive health and rights are concerned, especially for its highly appraised liberal abortion law in the 1996 Constitution, and its Choice on Termination of Pregnancy Act, 92 of 1995 (CTOPA) [Act 92 of 1995]. The Act allows provision of TOP on request up to the twelfth week of pregnancy, under broadly specified circumstances from the thirteenth to the twentieth week, and only for serious medical reasons after the twentieth week [Haroz, 1997]. It was amended in 2008 under the Choice on Termination of Pregnancy (CTOP) Amendment Act (No. 1 of 2008), and included specific reference to adolescents as also having the legal right to TOP [CTOPA, 2008]. As promising and robust as CTOPA is, its full implementation encountered a number of challenges, including unwilling healthcare providers due to their cultural and religious beliefs against TOP [Cooper et al., 2014; Harries et al., 2014; Harries et al., 2012; Harries et al., 2011; Bateman, 2011; Harrison, 2010; Mamabolo et al., 2010]. SA is a strongly traditional and religious country, with widespread opposition to abortion based on religious beliefs. Consequently, many adolescents are left to either carry a pregnancy they did not plan for or want, or undergo unsafe and illegal abortion.

Thus, many women, especially adolescents, experience difficulties in freely accessing TOP 
services because of stigma from healthcare workers, but also from the facility personnel [Bateman, 2011; Jewkes et al., 2005; Jewkes et al., 2002]. Adolescents often find themselves being reprimanded for being "sexually" irresponsible. As progressive as the reproductive health rights and policies are in SA, adolescents still face difficulties accessing the services and hence, teenage pregnancy for example has not been ceased under these comprehensive policies the country boasts. The adolescent sexual and reproductive health and rights (ASRH\&R) framework of 2015 further reports lack of knowledge about legal rights relating to sexual health and risks, particularly on TOP and emergency contraception (EC) among adolescents, highlighting the challenges adolescents face with their SRH [DSD, 2015].

There has been, however, a growing global recognition of the need to make adolescents' SRH services more friendly and accessible. The WHO advocates a 'quality of care' framework to make existing services accessible, acceptable, equitable, appropriate and effective for adolescents [WHO, 2015]. The framework emphasizes three essential components: 1) service providers must be trained about the needs of adolescents and youth, how to communicate and counsel them effectively, and how to provide services in supportive, non-threatening ways; 2) facilities must be friendly and welcoming, of good quality and acceptable to adolescents and youth, must protect their privacy and confidentiality and must be offered at subsidized rates or for free; and 3) more effective interventions must engage the community and make specific efforts to inform adolescents and youth about the services available for them [WHO, 2015]. Thus, for adolescents' SRH needs to be met and improved quality of life, enhanced efforts to maximize accessibility and availability of SRH services are needed as people with a healthy sex life and reproductive system have better quality of life [Waldman et al., 2015].

\section{Sexual and reproductive health consequences during adolescence}

Adolescence is regarded as the stage between 10-19 years of age, where physiological development and experiential behaviours are heightened. Adolescents are the biggest part of the total population ever in the world at about 1.3 billion as of 2012 [WHO, 2015b, UNICEF, 2012b]. Given the experiential nature of this population, adolescents are often at higher risk for a number of unpleasant health outcomes due to their typical risky behaviours in general. Adolescents' risky behaviours include, amongst others, alcohol and 
substance use, and early sexual debut, including unsafe sex practices, which could result in a series of negative health consequences. For example, unsafe sex could result in contracting an STI, which further increases the chances of contracting HIV infection; or result in an unplanned or unwanted pregnancy, which could lead to unsafe and illegal abortion.

With regards to HIV infection, 1,3 million adolescent girls worldwide are estimated to be living with HIV compared to 780,000 adolescent boys, demonstrating the heightened risks adolescent girls are faced with [UNICEF, 2012; UNAIDS, 2010]. Young people account for about $41 \%$ of new HIV infections every year, with $80 \%$ of these infections occurring among youth in SSA [UNAIDS, 2010]. Furthermore, 11-15\% of adolescent girls aged 15-19 years are reported to experience STIs compared to 2-11\% of boys [Santhya et al., 2015].

A pregnancy occurring during adolescence or between the ages of 15 to 19 years is termed a teenage pregnancy. Teenage pregnancy continues to be a global public health concern because of its frequent and serious medical and psychosocial consequences. Globally, about 16 million girls between the ages of 15 and 19 years give birth every year, translating to $11 \%$ of all births [WHO, 2015]. Almost all these births (95\%) occur in LMICs with sub-Saharan Africa (SSA) contributing by more than 50\% to global births [WHO, 2014]. In 2013, SSA had the highest prevalence of teenage pregnancy in the world [UNPFA, 2013].

About 7.4 million adolescent girls worldwide are at risk for unwanted pregnancies each year and of these, about 3 million experience unsafe abortions [WHO, 2011]. In the highincome countries (HICs), Romania had the highest rate of teenage pregnancy at 61 per 1000 women aged 15-19 in 2011. The United States (US) teenage pregnancy rates were 57 per 1,000 in 2010, and still remains the highest in the developed world, followed by New Zealand at 51 per 1000 women aged 15-19 years. England had the highest teenage pregnancy rates in Europe, as well as in the United Kingdom (UK) region at 47 per 1000 adolescent girls in 2011 [Santelli et al., 2013].

Teenage pregnancy rates in LMICs are more than double compared to that of the HICs. In SSA, the rates of teenage pregnancy differ within the region itself where some countries 
have much higher rates than others. For example, Burkina Faso had the highest teenage pregnancy rate in SSA at 187 per 1000 adolescent girls, while the rate in Ethiopia was at 121 per 1000 adolescent girls in 2008 [Guttmacher Institute]. About 10\% of adolescent girls in LMICs become mothers by the age of 16 years, with this rate being much higher in SSA. Again, this rate is different between the SSA countries where it is found to be $12.2 \%$ in Mozambique for instance, while it is $0.3 \%$ in Rwanda.

With these high rates of teenage pregnancy in SSA in general, follows the high rates of abortions, especially unsafe and illegal abortions. Globally, 2.5 million unsafe abortions are recorded among adolescent girls aged 15-19 years, with nearly $14 \%$ of these occurring in LMICs. Consequently, complications of unsafe and illegal abortions are vast among adolescent girls compared to older child-bearing women. Unsafe and illegal abortions pose a significant threat to adolescents' sexual and reproductive health and throughout their adulthood. Moreover, teenage pregnancy is also a contributing factor to maternal morbidity and mortality globally [WHO, 2014].

SA is amongst the countries in SSA where teenage pregnancy rates still remain unacceptably high despite the decrease observed in the past few decades, as a result of progressive social and educational policies, as well as accommodating reactions from families with regards to SRH education of teenagers (Mkhwanazi, 2014; Grant et al., 2008). In SA, about $40 \%$ of teenagers have given birth by the age of 20 and one in five 18 year old teenagers have given birth, highlighting the fact that young people in SA continue engaging in risk-taking behaviors including early and unsafe sexual activities (WHO, 2014; Grant \& Hallman, 2008). According to a review on pregnancy rates in SA, a rate of 71 per 1000 adolescent girls aged 15-19 years was reported [Panday et al., 2009]. The last Youth Risk Behaviour Survey (YRBS) of 2011 reported a 22.2\% of adolescent girls who ever had sex to have been pregnant [Reddy et al., 2011].

Pregnancy during adolescence is a risk for maternal mortality, including infant and child mortality compared to a pregnancy of older women due to a number of reasons, such as incomplete physiological development and, that their SRH needs and rights still remaining non-prioritized in many SSA countries [WHO, 2014; UNPFA, 2013]. Globally, the second most common cause of death among adolescents aged 15-19 years is complications during pregnancy and childbirth, with adolescents below the age of 16 
years at the highest risk for these complications [WHO, 2014]. In LMICs, infants born to mothers under 20 years of age face a 50\% higher risk of being still born or dying in the first few weeks versus those born to mothers aged 20-29 [WHO, 2014; Neal et al., 2015].

Birth outcomes of adolescent mothers are particularly poor with their newborns more likely to have low birth weight, accompanied by high risks of long-term effects such as early onset of adult diabetes [Martin et al., 2010; Jewkes et al., 2009; Chikonga et al., 2007]. Furthermore, adolescent mothers are at high risk for poor postpartum outcomes such as postpartum bleeding, anaemia, gestational hypertension, pre-eclampsia and eclampsia, and obstetric fistula [Neal et al., 2015; Ganchmeg et al., 2014; WHO, 2014; UNFPA, 2014; UNICEF, 2008; Conde-Agudelo, et al., 2005]. Yet, there is very little research conducted under the specific field of adolescents as vulnerable group and at higher risk for maternal mortality compared to older women during pregnancy, and there are also few studies focusing on healthcare provider perspectives regarding contraceptive use among adolescents, including the consequences of teenage pregnancy [Banke-Thomas, et al., 2017; Rankin et al., 2016].

Besides the health consequences of teenage pregnancy, psychosocial consequences also affect adolescents, sometimes throughout their adult life. Such psychosocial consequences include mental disturbances, reduced future employment opportunities, as many adolescents are often forced to dropout from school in order to care for their newborns [Neal et al., 2015; WHO, 2014; Chandra-Mouli et al., 2013; Black et al., 2012; Neal et al., 2012, Jewkes et al., 2009].

Thus, early childbearing increases the risks for maternal and neonatal mortality and therefore should be prevented [Neal et al., 2015]. Additionally, there is a clear need for studies focusing on promoting contraceptive use specifically in the SRH services for adolescents to prevent pregnancy [Rankin et al., 2016]. Patton et al. [2016] further emphasizes the benefits on investing in adolescents as that has a great potential to improve their lives into the next generation, creating better economic conditions. Given the potential dangers adolescents place themselves under by engaging in risky sexual behaviours and the detrimental consequences their reproductive health faces, adolescents' SRH services and needs cannot be overly emphasized. 


\section{Adolescents' access to and utilization of sexual and reproductive healthcare}

services

Despite the global agreements on adolescents' sexual and reproductive health and rights described above, access to and utilization of these services still remain unsatisfactory. More than 220 million women have an unmet need for FP in LMICs, with the majority being adolescents [Singh et al., 2012]. In SSA access to and utilization of SRH is particularly poor, especially the use of contraceptives (FP) [Tilahun et al., 2012; Jewkes et al., 2009; Biddlecom et al., 2007].

Many factors contribute to this poor access to and utilization of SRH services by adolescents. Such factors include, amongst others, social stigma surrounding the use of contraceptives for instance, fears of being perceived as promiscuous, limited information on SRH information and services, restricted school access for SRH services, judgmental attitudes and negative behaviours of healthcare workers, and other healthcare systems' related factors such as the shortage of healthcare professional [Jewkes et al., 2009; Chigona et al., 2007; Wood et al., 2006]. Additionally, shortage of resources (e.g., the lack of essential drugs) is also one of the reasons deterring people in general to seek healthcare services in public healthcare facilities in many regions in SSA [Ganle et al., 2014; Moyer et al., 2013]. This shortage is also applicable to contraceptives in some public clinics, and is likely to discourage people to continue seeking the service, posing a further barrier to access.

Clearly, a lot of key factors and stakeholders play an important role in the accessing of and utilization of SRH services by adolescents. In this thesis, the focus will be on healthcare workers as the key players in adolescents' SRH services. Healthcare workers, particularly primary healthcare nurses, are at the forefront in SRH services. Therefore, their role in SRH services and how they conduct themselves towards adolescents seeking SRH services is important to ascertain that adolescents receive the services they need without any prejudice from healthcare workers. However, research shows that healthcare workers are often seen as barrier to accessing care by young people, especially adolescents seeking SRH services because of their negative attitude and unfriendly behaviours [Morris et al., 2015; Geary et l., 2014; Ngoman et al., 2012; Chaibva et el., 2010; Wood et al., 2006]. 
Negative attitude of healthcare workers towards adolescents particularly, including the stigmatized adolescent sexual activity by many healthcare workers, is reported to affect utilization of SRH services, such as antenatal care, and contraceptives services [Ngoman et al., 2012]. Moreover, adolescents under-utilize SRH services because they fear being scolded and shouted at by healthcare workers [Geary et l., 2014; Wood et al., 2006] as the negative behaviours of healthcare workers are reported to discourage young people from attending clinics or for follow-up visits [Biddlecom et al., 2007; Glasier et al., 2006]. Surprisingly, healthcare workers' negative attitudes and behaviours towards adolescents seeking SRH services, are amongst the factors under-studied for potential to influence adolescents' access to and utilization of SRH services, including the use of contraceptives. Therefore, studying healthcare workers' behaviours and related personal determinants in SRH services seems particularly needed and important to understand, and to identify areas of improvement in order to enhance adolescents' access to and utilization of SRH services, especially the use of contraceptives to help reduce the high teenage pregnancy rates in the country.

\section{Aim and objectives}

The aim of this thesis is to identify the healthcare workers' behaviours and personal determinants affecting adequate provision of SRH to adolescents and how these affect access to and utilization of SRH services. In order to reach this aim, specific objectives were formulated:

(1) To identify adolescents' risky sexual and reproductive behaviours that continue to place them at risk for teenage pregnancy: the epidemiological study described in Chapter 2

(2) To identify the healthcare workers' behaviours and personal determinants affecting adequate quality provision of SRH services: the systematic review described in Chapter 3

(3) To identify the predictors of adequate quality SRH services provision to adolescents: the predictors'study described in Chapter 4

(4) To gain an understanding of healthcare workers perspectives with regards to adolescents' access to and utilization of SRH services: healthcare workers beliefs, behaviours and motivations study described in Chapter 5

(5) To identify healthcare workers' perceptions of adolescents seeking SRH services: the perceptions' study described in Chapter 6 
(6) Finally, to shed light into what psychosocial intervention programmes SRH healthcare workers need in order to enhance adolescents' access to and utilization of SRH services, in order to improve adolescents' reproductive health outcomes and ultimately help reduce teenage pregnancy in SA: described under general discussion in Chapter 7 


\title{
CHAPTER 2
}

\section{Published as:}

Jonas K, Crutzen R, van den Borne B, Sewpaul R, Reddy P. Teenage pregnancy rates and associations with other health risk behaviours: a three-wave cross-sectional study among South African school-going adolescents.

\begin{abstract}
Background: Teenage pregnancy still remains high in low and middle-income countries (LMIC), as well as in high-income countries (HIC). It is a major contributor to maternal and child morbidity and mortality rates. Furthermore, it has social consequences, such as perpetuating the cycle of poverty including early school dropout by the pregnant adolescent, especially in sub-Saharan Africa (SSA). Few studies in SSA have investigated the trends in teenage pregnancy and the associated factors, while this is critical in fully understanding teenage pregnancy and for promotion of reproductive health among adolescents at large in SSA.
\end{abstract}

Methods: To examine the trends in teenage pregnancy and to identify associations with other health risk behaviours in South Africa (SA), a total of 31816 South African schoolgoing adolescents between 11 to 19 years of age were interviewed in three crosssectional surveys. Data from the first (2002, $n=10549)$, second $(2008, n=10270)$ and the third (2011, $n=10$ 997) nationally representative South African youth risk behaviour surveys (YRBS) were used for this study.

Findings: The overall prevalence of having ever been pregnant among the combined 3survey sample was self-reported to be $11.0 \%$ and stable across the three surveys. Sexual intercourse among adolescents in SA has decreased from 41.9\% in 2002 to $36.9 \%$ in 2011. However, pregnancy among girls who ever had sex increased from $17.3 \%$ (95\% CI: $0.16-0.19$ ) in 2002, to $23.6 \%$ (95\% CI: $0.21-0.26$ ) in 2008 and decreased to $21.3 \%$ (95\% CI: $0.19-0.23$ ) in 2011. The odds for ever been pregnant were higher for girls who had 2 or more sexual partners (OR: 1.250, 95\% CI: 1.039 - 1.503), girls who ever used alcohol before sex (OR: 1.373, 95\% CI: 1.004 - 1.878), practised binge-drinking during the last month (OR: 0.624, 95\% CI: $0.503-0.774$ ), and girls who used mandrax (OR: 1.968, 95\% CI: 1,243 - 3.117). The odds for never been pregnant were lower for those who used condoms (OR: 0.462, 95\% CI: 0.309 - 0.691).

Conclusion: Girls continue to become pregnant at unacceptably high rates in SA. Sexual intercourse among adolescents in SA has decreased slightly. However, among those who 
are sexually active pregnancy prevalence rates have increased. More over, this is in the context of high prevalence of HIV and other STI. There is a need to address adolescents' sexual and reproductive health, and several health risk behaviours, including substance use, that are associated with teenage pregnancy in SA. 


\section{Introduction}

High teenage pregnancy rates are reported in both LMICs and HICs. The rate of teenage pregnancy in the United States is among the highest (at about 24\%) of the HICs while more than $50 \%$ of all births occurring during adolescence are in sub-Saharan Africa [Martin et al., 2012; WHO, 2015]. Globally, almost 16 million girls aged 15 to 19 give birth every year WHO, 2015 and about 2.5 million of these births occur to girls aged under 16 in LMICs each year [Neal et al., 2012]. This is about 11\% of all births worldwide, with the majority of these (95\%) occurring in LMIC WHO, 2015. Complications during pregnancy and childbirth worldwide are the second leading cause of death for 15-19 year-old girls, with girls below 16 years of age at a higher risk for maternal mortality and severe morbidity in comparison with women above the age of 20 years [WHO, 2015, Neal et al., 2012; Neal et al., 2013; Ganchimeg et al., 2014; Jewkes et a., 2009]. In addition, birth outcomes for very young adolescent mothers are particularly poor in terms of increased rates of low birth weights [Jewkes et al., 2009; Chikonga et al., 2007]. Furthermore, teenage pregnancy is a major contributor to a never ending cycle of ill-health and poverty worldwide [WHO, 2015; Neal et al., 2013; Ganchimeg et al., 2014; Jewkes et al., 2009].

Teenage pregnancy in SA is a multifaceted problem with many contributing factors such as poverty, gender inequalities, gender-based violence, substance use, poor access to contraceptives and issues with termination of pregnancy; low, inconsistent and incorrect use of contraceptives, limited number of healthcare practitioners and healthcare facilities, poor healthcare workers' attitudes and behavior, and inadequate sexual and reproductive health (SRH) information [Jewkes et al., 2009; Chikonga et al., 2007; Bearinger et al., 2007; Pettifor et al., 2005]. Besides these structural and healthcare systems related factors, adolescents in general tend to engage in risky behaviours including sexual activities that eventually put them at risk for unwanted pregnancies, sexually transmitted infections (STI's), and HIV Arnett, 2000. Sexual risk-taking behaviours, including early sexual debut, unprotected sex, multiple sex-partners and low contraceptive use are common among young people in SA [Arnett, 2000; Atuyambe et al., 2015; Wood et al., 2006] even though the SA government has made the provision of contraceptives free since 2001. The government continues to update and upgrade the scope of contraceptives in the country, including the addition of the very modern forms of contraception such as implants that are available in the public state hospitals and in primary healthcare clinics 
since 2014 [Schneider et al., 1999; Wilkinson et al., 2001; DoH, 2002]. Since 1994, emergency contraceptives and maternal and child healthcare services are free of charge while termination of pregnancy (TOP) has been legal and is also provided free of charge since 1996.

There exists a large body of literature worldwide on factors associated with teenage pregnancy and early childbearing both in HIC and in LMIC, such as low levels of contraceptive use. For example, a study on contraceptive use among adolescents in Kenya reported the use to be low, despite the reported high levels of knowledge about contraceptives among this population [Ochako et al., 2015]. In SA, a few studies have also reported on the factors associated with teenage pregnancy and other sexual risk behaviours such the low use of contraceptives and poor knowledge of contraceptives, amongst others mentioned above [Wood et al., 2006; Vundule et al., 2001; Eaton et al., 2003; Bhana et al., 2010; Mkhwanazi et al., 2010]. The reproductive health ignorance among young people, such as the basic understanding of a pregnancy possibility as a consequence of unsafe sexual intercourse, is also contributing to teenage pregnancy [Macleod, 1999]. Unsafe sex is common among South African adolescents and is known to result in a number of unpleasant health and social consequences [Pettifor et al., 2005; Wood et al., 2006; Mkhwanazi et al., 2010; Macleod, 1999; Makiwane et al., 2006; Shisana et al., 2002; Grant et al., 2008]. Teenage pregnancy in SA is on top of the list of consequences of unsafe sex, following HIV and AIDS, and other STI's.

Sexual risk behaviors and substance use often occur in combination with one another, both in developed countries as well as in LMIC [Kirby et al., 2001; Cavazos-Rehg et al., 2011; Palen et al., 2006; Taylor et al., 2003]. Substance use, particularly marijuana and cocaine, continue to represent individual-level risk factors associated with adolescents sexual behaviors, including use of contraception, safe sex practices, and teenage pregnancy [Kirby et al., 2001]. Furthermore, substance use among adolescents, especially alcohol and drugs has been reported to influence what adolescents do sexually, and makes them less likely to practice safe sex [Cavazos-Rehg et al., 2011]. Substance use behaviors in general, independently contribute to an increased risk in sexual intercourse experience with and without a history of teenage pregnancy [Kirby et al., 2001; CavazosRehg et al., 2011]. A dose-response relationship between an increased likelihood of a teenage pregnancy and both marijuana use and daily cigarette smokers who initiated use 
at age 12 or younger was reported among adolescents in the United States (US) [CavazosRehg et al., 2011]. Substance use in SA has been associated with higher odds of lifetime sexual intercourse [Palen et al., 2006]. High school adolescents who used alcohol or smoked cigarettes were two to three times more likely to be sexually active in the KwaZulu-Natal province [Taylor et al., 2003].

There is clearly a lot of literature on teenage pregnancy, but its scope is limited to the causes of teenage pregnancy [Macleod, 1999], the implications of pregnancy to the young mother (and her baby) [Makiwane et al., 2006; Kaufman et al., 2001], and to school dropout related factors [Makiwane et al., 2006; Grant et al., 2008]. There are however, very limited studies published that report on recent trends in teenage pregnancy in SA [Kaufman et al., 2001]. Even less is known about the factors associated with trends in teenage pregnancy. Analysis of teenage pregnancy trends and the associated factors is of paramount importance, as it will help to understand fully the factors surrounding teenage pregnancy in SA. It provides useful information towards understanding the magnitude of teenage pregnancy among the South African youth and helps determining areas that require serious intervention efforts in order to reduce the rates of teenage pregnancy. Understanding the factors or determinants associated with teenage pregnancy is crucial because it is this understanding that will enable development of intervention programmes needed to address teenage pregnancy in the country. Thus, the aim of this particular study is to examine the trends in teenage pregnancy as well as related health risk behaviors, including unsafe sex, substance use, partner violence, and psychological well-being; which might act as contributory factors associated with teenage pregnancy among school-going adolescents between 11 and 19 years of age in different settings of the country.

\section{Methods}

Data of the three Umthente Uhlaba Usamila: South African National Youth Risk Behaviour Surveys (YRBS) are used in this study. Three cross-sectional surveys were conducted among nationally representative samples of grades 8 to 11 secondary school adolescents in South Africa in 2002, 2008, and 2011. 


\section{Participants and Sampling}

The SA YRBS used a two-stage cluster sample design. At the first stage of sampling, schools were stratified according to the country's nine provinces. For each sample the most recent list of all public schools in the country, provided by the South African National Department of Education, was used as the sampling frame, to ensure nationally representative data. The schools were the primary sampling units and were selected with a probability proportional to the schools' adolescent enrolment in grades 8 to 11 . In each survey twenty-three schools per province were sampled and invited to participate. At the second stage of sampling, classes were randomly selected within each participating school. A total of 14766 (for 2002 survey), 13379 (for the 2008 survey) and 14387 (for the 2011 survey) enrolled adolescents from grades 8-11 in the participating schools per province were invited for each survey respectively. Nationally, a $72 \%$ response rate for 2002 and 2011, and 76\% for 2008 was achieved at the adolescent level.

Adolescents completed a self-administered questionnaire on a range of sociodemographic characteristics and risk behaviour variables. The YRBS questionnaire was adapted from the US Youth Risk Behaviour Survey, a health survey conducted biennially among high school adolescents in the United States [Troped et al., 2007]. The measures used in the study are specified below. Further details regarding the South African YRBS survey and methodology can be found elsewhere [Reddy et al., 2013; Reddy et al., 2008; Reddy et al., 2002; PubChem].

\section{Measures}

\section{Demographics}

Age: to determine the ages of adolescents in the sample, adolescents were asked to report both their age and date of birth.

Gender: adolescents were asked to report their gender ( $0=$ Male, $1=$ Female $)$.

Race: race was classified according to the South African Department of Labour designated categories: Black African $(=1)$, Coloured (mixed Black and White descent $(=2)$, Indian $(=3)$, White $(=4)$, or Other $(=5)$. 


\section{$\underline{\text { Socio-economic status }}$}

Fathers' employment status: if adolescents reported their father having a paying job 5 or more days a week $(=1)$, No, he gets a social grant $(=2)$, No, he is unemployed $(=3)$, No, he is ill or disabled $(=4)$, No, he has retired $(=5)$, My father is deceased (=6), I don't know $(=7)$.

Mothers' employment status: if adolescents reported their mother having a paying job 5 or more days a week $(=1)$, No, she is a housewife $(=2)$, No, she is unemployed (=3), No, she is ill or disabled $(=4)$, No, she has retired $(=5)$, My mother is deceased $(=6)$, I don't know $(=7)$.

\section{$\underline{\text { Sexual behaviours }}$}

Ever had sex: if adolescents reported having ever had sex (when the penis enters the vagina or anus) ( $1=$ Yes, $0=$ No). The following questions were dependent on whether the adolescent answered "yes" (1) on ever had sex, although there was no skip pattern for adolescents who answered "no" (0).

First had sex before age of 14 years: if adolescents reported having had first sex at the age of 13 years or younger $(1=$ had sex at $\leq 13$ years, $0=$ have never had sex or had sex at age 14 years or older).

Number of lifetime sexual partners: if adolescents reported having ever had sex with 1 or more people $(0=$ never had sex or 1 partner, $1=2$ or more partners). For the purposes of this study, lifetime sexual intercourse is defined as the number of times whereby one's penis had penetrated a vagina (or one's vagina ever been penetrated by a penis) since they were born. The number of lifetime sexual partners therefore, refers to the number of sexual partners one has ever had sexual intercourse with in their lifetime.

Used alcohol before sex: if adolescents reported having consumed alcohol before the last time they had sex ( $1=$ Yes, $0=$ No/ never had sex $)$.

Always or mostly used a condom during sex: if adolescents reported always using condoms when they have sex ( 1 = always or mostly using a condom before sex, $0=$ never had sex or not always using a condom during sex or never using a condom during sex).

Contraceptive methods mostly used to prevent pregnancy: if adolescents reported using contraceptives $(0=$ No method, $1=$ Birth control pills, $2=$ Condoms, $3=$ Contraceptive injection, $4=$ Other).

Ever been pregnant: if adolescents reported to ever been pregnant $(1=\mathrm{Yes}, 0=\mathrm{No} /$ never had sex). 
Ever had an abortion: if adolescents reported to ever had an abortion (1= Yes, $0=$ No/never sex/never pregnant).

\section{Violent behaviours}

Ever been hit by boyfriend or girlfriend: if adolescents reported that, during the past six months, their boyfriend or girlfriend hit, smacked or physically hurt them on purpose, then they were classified as being physically hurt by boyfriend or girlfriend $(1=$ Yes, $0=$ No).

Ever hit your boyfriend or girlfriend: if adolescents reported that, during the past six months, they have hurt their boyfriend or girlfriend, smacked or physically hurt them on purpose, then they were classified as having physically hurt their boyfriend or girlfriend $(1=$ Yes, 0 = No).

Ever been forced to have sex: if adolescents reported that they had ever been physically forced to have sex when they did not want to, then they were classified as such $(1=$ Yes, 0 $=$ No).

Ever forced someone to have sex: if adolescents reported that they had ever physically forced someone to have sex when they did not want to, then they were classified as such $(1=$ Yes, $0=$ No) .

\section{$\underline{\text { Substance use behaviours }}$}

Current cigarette smoking: Adolescents were categorised as being current cigarette smokers if they reported having smoked cigarettes on at least one day in the past month (1= Yes, 0 = No).

Alcohol use: Adolescents were categorised as being alcohol users if they reported having ever drank one or more drinks of alcohol ( $1=$ Yes, $0=$ No) in their lifetime.

Binge drinking in the past month: Adolescents were categorised as having engaged in binge drinking if they reported having had five or more drinks of alcohol (e.g. a beer, a glass of wine, or a 'tot' of brandy) in a row on at least one day in the past month $(1=$ Yes, 0 $=$ No).

Marijuana use: if adolescents reported ever having smoked marijuana at least once in their lifetime ( $1=$ used marijuana on at least their lifetime, $0=$ did not use marijuana in their lifetime).

Ever used hard drugs: if adolescents reported having ever used any one of the following drugs; sniffed glue, mandrax, cocaine, heroin, injection drugs, and any other illegal drug 
(1= ever used the drug, $0=$ never or did not use the drug) for each of the drugs. Methaqualone is sold under the brand name Quaalude in the US and Mandrax in the UK and South Africa. It was used to reduce anxiety and tension when it was legal as a sedative and hypnotic drug. Due to its addictive nature, it is now illegal for medical use and commonly used by recreational drug users [PubChem]. Mandrax is amongst the frequently abused drugs used by drug users in SA, hence its inclusion in this study.

\section{Psychological well-being}

Have you had serious sad and hopeless feelings in past six months: if adolescents reported that they felt so sad that they stopped doing their usual activities for two weeks or more in $\operatorname{row}(1=$ Yes, $0=$ No $)$.

Suicidal: if adolescents reported that they have thought or made a plan about how to attempt suicide during the 6 months preceding the survey $(1=$ Yes, $0=$ No).

\section{Ethical approval and consent}

Ethical Approval for the study was obtained from the South African Medical Association Research Ethics Committee. Active informed consent to conduct the study was obtained from the National Department of Education, school principals, parents and learners. In addition, assent was also obtained from learners on the day of the study. Learners were requested not to write their names on the answer sheet to ensure their anonymity. To ensure learners' confidentiality, only trained survey administrators were allowed to remain with the learners while completing the survey. School teachers were requested to leave the classroom during data collection time. Learners were also requested not communicate with each other or look at the answer sheet of their peers during the completion of the survey. Information sheet regarding the surveys was also provided to the learners.

\section{Analyses}

Frequency data were weighted to approximate province level distributions of gender and grade, and to account for non-response and province size. Weights were post-stratified by grade and gender, so that the weighted counts of students in each grade and gender combination were in proportion to the provincial population proportions. Each province was to be represented equally in the sample. To account for the variations in provinces' total population, respondents in highly populated provinces had to have higher weights 
than respondents from less populated provinces. The three different YRBS data sets were combined for the trend analysis purposes. The trend analysis followed the protocol on Conducting Trend Analysis of YRBS data published by the US Center for Disease Control [CDC, 2014]. Descriptive statistics were first explored to gain a clearer picture of the data as well as to summarize the characteristics of the overall sample. The combined dataset was then split according to the respective years. Frequencies were run for each survey to provide overview differences in characteristics for the three surveys. This provided insight into trends in teenage pregnancy and sexual activity behaviours among adolescent who reported to have ever had sex in the three different surveys. Trends were considered significant if 95\% confidence intervals did not include 1 and the p-value was below 0.05 . Bivariate correlation analysis was performed to establish risky behaviours associated with teenage pregnancy.

A binary logistic regression analysis was used to examine unique associations between the demographic, substance use, violence, and sexual and suicide related behaviour variables, and the primary outcome measure: ever been pregnant. Collinearity statistics showed the variables taken into the logistic regression model did not violate the multicollinearity assumptions: all the variables in the model were below the cut off value for the variance inflation factor (VIF) of 10 [Pallat, 2013; Field, 2009]. The adjusted relationships of these measures for ever been pregnant were modelled in a binary logistic regression model. A significance level of 5\% was used for all analyses. IBM SPSS Version 21.0 was used to analyse the data. Description of sexual risk behaviours, bivariate correlations, and the logistic regression analysis (Table 2-4) only included adolescent girls $(\mathrm{N}=5060)$ who reported to have ever had sex.

\section{Results}

\section{Demographics}

A total of 31816 school-going adolescents were surveyed during the three surveys (2002, 2008, and 2011). The combined sample consisted of $47.6 \%$ males and $52.4 \%$ females between the ages of 11 to 19 years of age, with (78.4\%) being Black Africans, (13.0\%) Coloured, (1.3\%) Indian, (6.3\%) White, and (1.0\%) other. Less than half of the adolescents have a father (39.0\%) or a mother (31.3\%) who had a paid job. 


\section{Sexual behaviours among adolescents}

There has been a decrease in sexual intercourse between the adolescents surveyed in 2002, 2008, and those surveyed in 2011 (41.9\%, 95\% CI: $0.41-0.43 ; 37.6 \%, 95 \%$ CI: 0.36- 0.37; and 36.9\%, 95\% CI: 0.35 - 0.37; respectively) and overall 38.8\%, reported to have ever had sex in the combined sample.

Table 1 shows descriptive characteristics of boys and girls, by year and for the combined sample. There are notable differences on ever had sex between boys and girls throughout the survey years. About $51 \%$ of boys in 2002 reported to ever had sex compared to $34.0 \%$ of girls in the same survey year. Sex among boys and girls in the study continued to decrease from 2002 50.9\%, (95\% CI: 0.48 - 0.50), 2008 45.1\%, (95\% CI: 0.53 - 0.56), to 2011 45.2\%, (95\% CI: 0.53 -.0.56) for boys; and 34.0\%, (95\% CI: 0.65 - 0. 67); 30.1\%, (95\% CI: 0.69 - 0.71); 29.8\%, (95\% CI: 0.69 - 0.71) for girls in 2002, 2008, and 2011, respectively. In the combined sample similar proportions of both boys (11.1\%), and girls (10.9\%) reported to ever making someone pregnant for boys or ever having been pregnant in the case of girls. Overall, $11.0 \%$ of the adolescents reported to ever made someone pregnant or ever been pregnant in this study. 
Table 1: Descriptive characteristics of the total sample ${ }^{1}$ by year

\begin{tabular}{|c|c|c|c|c|c|c|c|c|c|c|c|c|}
\hline \multicolumn{5}{|c|}{ BOYS } & \multicolumn{4}{|c|}{ GIRLS } & \multicolumn{4}{|c|}{ Total sample } \\
\hline Characteristics & $\begin{array}{l}2002 \\
(\mathrm{~N}=4930) \\
\%\end{array}$ & $\begin{array}{l}2008 \\
(N=5106) \\
\%\end{array}$ & $\begin{array}{l}2011 \\
(\mathrm{~N}=5166) \\
\quad \%\end{array}$ & $\begin{array}{l}\text { TOTAL } \\
\text { BOYS } \\
(\mathrm{N}=15202) \\
\quad \%\end{array}$ & $\begin{array}{l}2002 \\
(\mathrm{~N}=5619) \\
\quad \%\end{array}$ & $\begin{array}{l}2008 \\
(N=5164) \\
\%\end{array}$ & $\begin{array}{l}2011 \\
(\mathrm{~N}=5831) \\
\%\end{array}$ & $\begin{array}{l}\text { TOTAL } \\
\text { GIRLS } \\
(\mathrm{N}=16614) \\
\quad \%\end{array}$ & $\begin{array}{c}2002 \\
(\mathrm{~N}=10549) \\
\%\end{array}$ & $\begin{array}{c}2008 \\
(N=10270) \\
\%\end{array}$ & $\begin{array}{c}2011 \\
(N=10997) \\
\%\end{array}$ & $\begin{array}{c}\text { ALL } \\
(\mathrm{N}=31 \\
816) \\
\%\end{array}$ \\
\hline Age & & & & & & & & & - & - & - & - \\
\hline Mean & 16.9 & 16.1 & 16.6 & 16.6 & 16.4 & 16.1 & 16.3 & 16.2 & 16.6 & 16.1 & 16.4 & 16.4 \\
\hline Standard deviation & 3.3 & 1.9 & 2.0 & 2.5 & 2.6 & 1.9 & 1.9 & 2.2 & 2.9 & 1.9 & 1.9 & 2.3 \\
\hline \multicolumn{13}{|l|}{ Gender } \\
\hline Male & - & - & - & - & - & - & - & - & 46.7 & 49.1 & 47.0 & 47.6 \\
\hline Female & - & - & - & - & - & - & - & - & 53.3 & 50.9 & 53.0 & 52.4 \\
\hline \multicolumn{13}{|l|}{ Race } \\
\hline Black African & 74.7 & 78.7 & 82.4 & 78.7 & 73.5 & 79.1 & 82.3 & 78.3 & 74.1 & 78.5 & 82.4 & 78.4 \\
\hline Coloured & 14.4 & 13.7 & 10.1 & 12.6 & 15.6 & 14.8 & 10.0 & 13.4 & 15.0 & 14.2 & 10.0 & 13.0 \\
\hline Indian & 1.4 & 0.9 & 1.4 & 1.2 & 1.2 & 0.8 & 1.3 & 1.1 & 1.3 & 1.2 & 1.4 & 1.3 \\
\hline White & 8.4 & 6.0 & 4.7 & 6.4 & 8.8 & 4.4 & 5.3 & 6.2 & 8.7 & 5.3 & 5.1 & 6.3 \\
\hline Other & 1.1 & 0.7 & 1.4 & 1.1 & 0.9 & 0.9 & 1.1 & 0.9 & 0.9 & 0.8 & 1.2 & 1.0 \\
\hline \multicolumn{13}{|l|}{$\begin{array}{l}\text { Socio-economic } \\
\text { status }\end{array}$} \\
\hline $\begin{array}{l}\text { Father has paid job } \\
5 \text { or more days a } \\
\text { week }\end{array}$ & 41.1 & 40.5 & 37.9 & 39.8 & 40.2 & 38.1 & 36.4 & 38.2 & 40.6 & 39.4 & 37.1 & 39.0 \\
\hline $\begin{array}{l}\text { Yes, works less than } \\
5 \text { days a week }\end{array}$ & 8.6 & 9.2 & 8.5 & 8.7 & 8.5 & 7.4 & 7.4 & 7.7 & 8.5 & 8.3 & 7.9 & 8.2 \\
\hline $\begin{array}{l}\text { No, he gets a social } \\
\text { grant }\end{array}$ & $*$ & 7.0 & 7.3 & 10.6 & $*$ & 7.3 & 7.3 & 10.7 & $*$ & 7.1 & 7.3 & 10.6 \\
\hline $\begin{array}{l}\text { No, he is } \\
\text { unemployed }\end{array}$ & 17.5 & 10.9 & 11.1 & 8.2 & 17.4 & 12.2 & 12.0 & 8.9 & 17.5 & 11.6 & 11.6 & 8.6 \\
\hline $\begin{array}{l}\text { No, he is ill or } \\
\text { disabled }\end{array}$ & 2.6 & 1.4 & 1.1 & 2.7 & 2.7 & 1.6 & 0.9 & 2.2 & 2.6 & 1.5 & 1.0 & 2.4 \\
\hline $\begin{array}{l}\text { No, he has retired } \\
\text { My father is }\end{array}$ & 5.5 & 3.5 & 3.0 & 7.6 & 4.1 & 2.7 & 2.3 & 7.9 & 4.8 & 3.1 & 2.6 & 7.7 \\
\hline deceased & 16.5 & 10.0 & 13.3 & 10.6 & 18.4 & 13.0 & 14.7 & 12.1 & 17.5 & 11.5 & 14.1 & 11.4 \\
\hline
\end{tabular}




\begin{tabular}{|c|c|c|c|c|c|c|c|c|c|c|c|c|}
\hline I don't know & 8.2 & 17.4 & 17.8 & 11.8 & 8.7 & 17.8 & 19.0 & 12.1 & 8.5 & 17.6 & 18.4 & 12.0 \\
\hline $\begin{array}{l}\text { Mother has paid job } \\
5 \text { or more days }\end{array}$ & 29.8 & 32.4 & 31.2 & 31.2 & 32.4 & 30.8 & 31.1 & 31.4 & 31.2 & 31.7 & 31.1 & 31.3 \\
\hline $\begin{array}{l}\text { Yes, works less than } \\
5 \text { days a week }\end{array}$ & 12.6 & 12.7 & 12.3 & 12.5 & 11.1 & 12.1 & 10.8 & 11.3 & 11.8 & 12.4 & 11.5 & 11.9 \\
\hline No, she gets a social & 23.3 & 9.5 & 10.7 & 14.4 & 24.8 & 11.6 & 12.1 & 16.3 & 24.1 & 10.5 & 11.4 & 15.4 \\
\hline $\begin{array}{l}\text { grant } \\
\text { No, she is a } \\
\text { housewife }\end{array}$ & 20.0 & 21.9 & 20.5 & 20.8 & 19.6 & 24.1 & 23.8 & 22.5 & 19.9 & 22.9 & 22.2 & 21.7 \\
\hline No, she is ill or & 2.1 & 2.0 & 1.7 & 2.0 & 1.9 & 1.8 & 1.7 & 1.8 & 2.0 & 1.9 & 1.7 & 1.9 \\
\hline $\begin{array}{l}\text { disabled } \\
\text { No, she has retired }\end{array}$ & 3.6 & 3.8 & 3.7 & 3.7 & 2.3 & 2.5 & 3.1 & 2.6 & 2.9 & 3.1 & 3.4 & 3.1 \\
\hline $\begin{array}{l}\text { My mother is } \\
\text { deceased }\end{array}$ & 4.8 & 5.5 & 7.4 & 5.9 & 5.1 & 6.5 & 7.8 & 6.5 & 5.0 & 6.0 & 7.6 & 6.2 \\
\hline I don't know & 3.6 & 12.2 & 12.5 & 9.5 & 2.8 & 10.6 & 9.7 & 7.6 & 3.2 & 11.4 & 11.0 & 8.5 \\
\hline \multicolumn{13}{|l|}{ Sexual behaviours } \\
\hline Ever had sex & 50.9 & 45.1 & 45.2 & 47.0 & 34.0 & 30.1 & 29.8 & 31.3 & 41.9 & 37.6 & 36.9 & 38.8 \\
\hline $\begin{array}{l}{ }^{1} \text { Ever } \\
\text { pregnant }\end{array}$ & 11.0 & 10.7 & 11.6 & 11.1 & 11.2 & 11.2 & 10.3 & 10.9 & 11.1 & 11.0 & 10.9 & 11.0 \\
\hline \multicolumn{13}{|l|}{ Violent behaviours } \\
\hline $\begin{array}{ll}\text { Ever been hit by } \\
\text { boyfriend } & \text { or } \\
\text { girlfriend } & \end{array}$ & 15.3 & 24.7 & 19.2 & 19.2 & 13.6 & 21.1 & 17.2 & 16.6 & 14.4 & 23.0 & 18.2 & 17.9 \\
\hline $\begin{array}{lr}\text { Ever hit your } \\
\text { boyfriend }\end{array}$ & 15.6 & 21.8 & 18.1 & 18.2 & 11.4 & 17.6 & 13.9 & 13.8 & 13.4 & 19.7 & 15.9 & 15.9 \\
\hline girlfriend & & & & & 10.7 & 8.7 & 8.3 & 9.2 & 9.8 & 9.9 & 9.4 & 9.7 \\
\hline $\begin{array}{l}\text { Ever been forced to } \\
\text { have sex }\end{array}$ & 8.7 & 10.9 & 10.7 & 10.1 & 6.3 & 6.4 & 5.1 & 5.9 & 7.9 & 9.0 & 8.0 & 8.3 \\
\hline $\begin{array}{l}\text { Ever forced } \\
\text { someone to have sex }\end{array}$ & 9.7 & 11.6 & 11.3 & 10.9 & & & & & & & & \\
\hline \multicolumn{13}{|l|}{ Cigarette smoking } \\
\hline 0 days & 67.1 & 71.5 & 75.4 & 71.4 & 79.9 & 83.1 & 86.1 & 83.1 & 73.9 & 77.2 & 81.1 & 77.5 \\
\hline 1 or more days & 32.9 & 28.5 & 24.6 & 28.6 & 20.1 & 16.9 & 13.9 & 16.9 & 26.1 & 22.8 & 18.9 & 22.5 \\
\hline
\end{tabular}




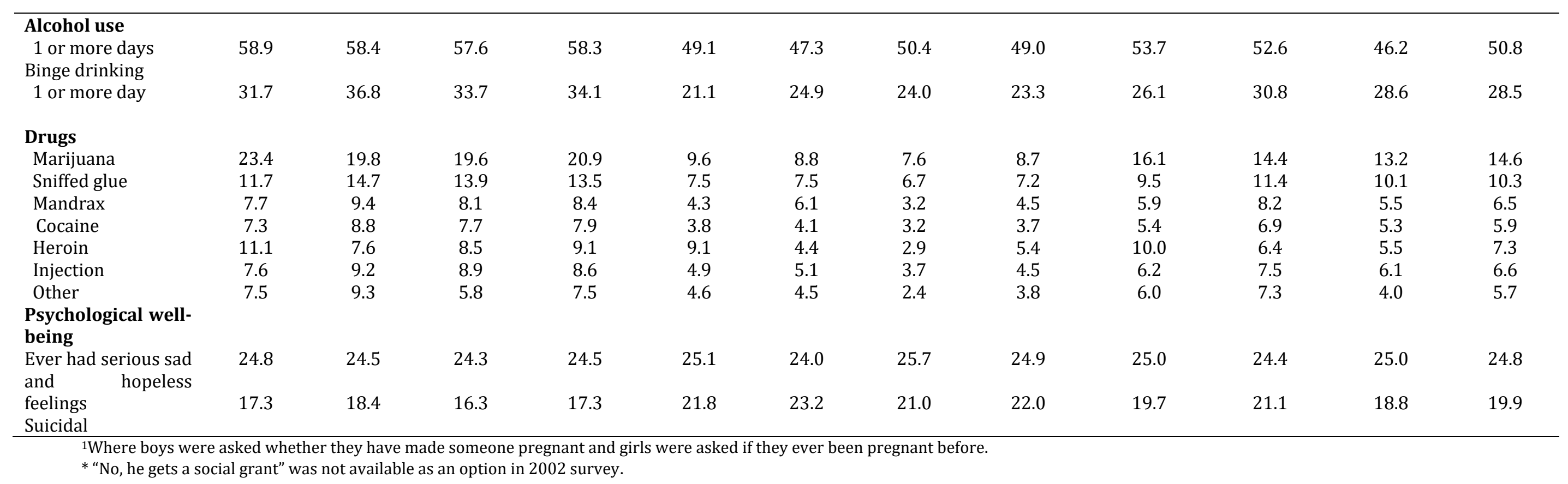




\section{Violent behaviours among adolescents}

About $17.9 \%$ of the adolescents reported having been hit on purpose by a boyfriend or girlfriend, while $15.9 \%$ reported to ever hit their boyfriend or girlfriend on purpose. During the three survey years, 9.8\%, 9.9\%, and 9.4\% of the adolescents reported that they had been forced to have sex, respectively. In the combined sample, $9.7 \%$ of adolescents reported having been forced to have sex, while $8.3 \%$ reported to have forced someone else to have sex.

\section{Substance use related behaviours among adolescents}

Ever smoked cigarettes was reported by $22.5 \%$ of the adolescents, ever having used alcohol by $53.4 \%$ and $28.5 \%$ admitted to binge drinking. Smoking dagga (marijuana) was reported by (14.6\%) adolescents, followed by sniffed glue (10.3\%), heroin $(7.3 \%)$, and injection drugs (6.6\%). Mandrax, cocaine and other drugs were the least used drugs by the adolescents at $6.5 \%, 5.9 \%$ and $5.7 \%$ respectively.

When comparing the different years, marijuana use decreased from 16.1\% (95\% CI: 0.15 - 0.17) in 2002, 14.4\% (95\% CI: $0.14-0.15$ ) in 2008 to $13.2 \%$ (95\% CI: 0.13 - 0.14) in 2011. The use of mandrax increased in 2008 to 8.2\% (95\% CI: $0.08-0.09$ ) from 5.9\% (95\% CI: 0.05 - 0.06) in 2002, although it decreased in 2011 to 5.5\% (95\% CI: 0.05 0.06). This pattern was also observed for the use of cocaine in 2002, 2008 and 2011 at 5.4\% (95\% CI: 0.05 - 0.06), 6.9\% (95\% CI: $0.06-0.07)$ and 5.3\% (95\% CI: $0.05-0.06)$; respectively.

Substance use between boys and girls were notably different. Nearly twenty nine per cent of boys (28.6\%) smoked cigarettes one or more days, while only $16.9 \%$ of girls smoked cigarettes. More boys (58.3\%) than girls (49.0\%) ever used alcohol in this study. Similarly with binge drinking during the past month, $34.1 \%$ boys and $23.3 \%$ girls reported binge drinking. When comparing the three surveys, there is a slight decrease in alcohol use from 2002 (53.7\%), 2008 (52.6\%) to 2011 (46.2\%). The overall use of alcohol in the combined sample is $50.8 \%$. The percentage of binge drinkers was $26.1 \%$ in 2002 , but increased in 2008 (30.8\%) and slightly decreased in 2011 (28.6\%). The overall percentage of binge drinking in the combined sample was $28.5 \%$. Marijuana, the drug most commonly used by the adolescents in this study was reported by $20.9 \%$ of the boys and by $8.7 \%$ of the girls. Sniffed glue (13.5\%), mandrax (8.4\%), cocaine $(7.9 \%)$, heroin $(9.1 \%)$, injection drugs 
(8.6\%), and other illegal drugs (7.5\%) were reported by the boys. While girls reported to have ever sniffed glue (7.2\%), used mandrax (4.5\%), cocaine (3.7\%), heroin (5.4\%), injection drugs (4.5\%), and other illegal drugs (3.8\%).

\section{Sexual risk behaviours and teenage pregnancy}

Table 2 below shows trends in sexual risk behaviours and teenage pregnancy among girls who reported to have ever had sex. The prevalence of ever been pregnant among the adolescent girls who ever had sex had increased from 17.3\%, (95\% CI: $0.16-0.19)$ in the 2002 survey, 23.6\%, (95\% CI: 0.21 - 0.26) in 2008, and slightly decreased in 2011 to 21.3\%, (95\% CI: $0.19-0.23)$. This shows a significant $(\mathrm{p}<0.01)$ increasing trend from the 2002 survey to the 2008 survey. The overall prevalence rate of ever been pregnant among the adolescent girls who reported to ever had sex was $20.5 \%$. Among those who reported ever had sex, 8.6\% in 2002, 6.6\% in 2008 and 7.4\% in 2011 had sex at the age of 13 years or younger, and with the overall being $7.7 \%$ when combining the three surveys. Sex with one person was reported by $68.2 \%$ of the girls, while $31.8 \%$ reported having had sex with 2 or more persons. When comparing the three surveys, $30.2 \%$ of the girls in $2002,28.7 \%$ in 2008, and $34.9 \%$ in 2011 reported to have sex with 2 or more persons.

Having sex under the influence of alcohol was reported by $6.7 \%$ of the girls in 2002, $13.1 \%$ in 2008 , and $13.8 \%$ in 2011 . The overall rate was $11.0 \%$ when all three surveys combined. This shows an increasing pattern of sexual intercourse under the influence of alcohol, as well as the use of alcohol. The prevalence of abortion was reported by $5.1 \%$ in $2002,6.8 \%$ in 2008 , and $6.0 \%$ in 2011 . The overall rate of condom use during sex was reported by $71.1 \%$ of the girls. Furthermore, condom use increased from $67.2 \%$ (95\% CI: $0.79-0.81$ ) in 2002 to $74.6 \%$, (95\% CI: 0.84- 0.85) in 2008, but decreased slightly in 2011 to $72.4 \%$ (95\% CI: 0.84 - 0.85). With regard to contraceptive methods used, condoms were among the most frequently used method compared to any other methods used at $40.7 \%$, while birth control pills were among the least used methods at $4.6 \%$. When comparing the surveys, $32.5 \%$ of the girls in $2002,44.3 \%$ in 2008 , and $46.6 \%$ in 2011 reported the use of condoms as the method for contraception. Use of birth control pills, on the one hand were reported by $4.6 \%, 3.8 \%$, and $5.2 \%$ in 2002, 2008, and 2011; respectively. The overall use of birth control pills in the combined sample was $4.6 \%$. On the other hand, injection use was reported by 14.4\%, 11.7\%, and 12.6\% in 2002, 2008, and 2011; respectively. The overall use of injection was $13.0 \%$ in the combined sample. In 
the combined sample, $14.6 \%$ reported no method used for contraceptive purposes.

Table 2: Sexual risk behaviours among adolescent girls who reported to ever had sex in their lives

\begin{tabular}{|c|c|c|c|c|}
\hline Risky behaviours & $\begin{array}{c}2002 \\
(\mathrm{~N}=1854) \\
\%\end{array}$ & $\begin{array}{c}2008 \\
(N=1499) \\
\%\end{array}$ & $\begin{array}{c}2011 \\
(\mathrm{~N}=1707) \\
\%\end{array}$ & $\begin{array}{c}\text { ALL } \\
(\mathrm{N}=5060) \\
\%\end{array}$ \\
\hline First had sex at age $\leq 13$ & 8.6 & 6.6 & 7.4 & 7.7 \\
\hline \multicolumn{5}{|l|}{ Number of sexual partners } \\
\hline 1 partner & 68.8 & 69.9 & 62.7 & 68.2 \\
\hline 2 or more partners & 30.2 & 28.7 & 34.9 & 31.8 \\
\hline $\begin{array}{l}\text { Ever use alcohol before sex } \\
\text { used condom during sex }\end{array}$ & 6.7 & 13.1 & 13.8 & 11.0 \\
\hline Never or rarely use condoms & 32.8 & 25.4 & 27.6 & 28.9 \\
\hline Mostly use condoms & 67.2 & 74.6 & 72.4 & 71.1 \\
\hline \multicolumn{5}{|l|}{ Contraceptive methods } \\
\hline No method & 16.0 & 14.2 & 13.3 & 14.6 \\
\hline Birth control pills & 4.6 & 3.8 & 5.2 & 4.6 \\
\hline Condoms & 32.5 & 44.3 & 46.6 & 40.7 \\
\hline Injection & 14.4 & 11.7 & 12.6 & 13.0 \\
\hline Other & 8.2 & 7.5 & 8.7 & 8.1 \\
\hline Ever been pregnant & 17.3 & 23.6 & 21.3 & 20.5 \\
\hline Ever had an abortion & 5.1 & 6.8 & 6.0 & 5.9 \\
\hline
\end{tabular}

\section{Association between teenage pregnancy and sexual risk behaviours}

The associations between the teenage pregnancy and risky behaviours were examined for those reported to have ever had sex. Table 3 below shows the results of the Pearson correlations between teenage pregnancy and the risky behaviours. There was a negative association between teenage pregnancy and condom use during sex $(r=-.084, p<0.01)$. This negative association, although weak, shows that when teenagers practise protective behaviours such as using condoms during sex; they are less likely to become pregnant.

The age at first sex, which is the age of 13 years or younger $(r=.056, p<0.01)$, and the number of sexual partners being two or more sexual partners $(r=.033, p<0.05)$, were positively correlated with teenage pregnancy. Ever been forced to have sex $(r=.032$, $p<$ $0.05)$, as well as ever forced someone to have sex $(r=.046, p<0.01)$, and been hit by boyfriend $(r=.048, p<0.01)$ were also positively correlated with teenage pregnancy. 
Table 3: Association between teenage pregnancy and sexual risk behaviours among adolescent girls who ever had sex $(\mathrm{N}=5060)$

\begin{tabular}{lc}
\hline \multicolumn{1}{c}{ Risky behaviours } & $\frac{\text { Ever been pregnant }}{(\mathbf{r})}$ \\
\hline & $.056^{* *}(95 \% \mathrm{CI}: 0.03-0.08)$ \\
First had sex at age $\leq 13$ & $.033^{*}(95 \% \mathrm{CI}: 0.01-0.04)$ \\
Number of sexual partners & $.012(95 \% \mathrm{CI}: 0.02-0.04)$ \\
Ever used alcohol before sex & $-.084^{* *}(95 \% \mathrm{CI}:-0.11--0.06)$ \\
Always used a condom during sex & $.032^{*}(95 \% \mathrm{CI}: 0.01-0.06)$ \\
Ever been forced to have sex & $.046^{* *}(95 \% \mathrm{CI}: 0.02-0.07)$ \\
Ever forced someone to have sex & $.048^{* *}(95 \% \mathrm{CI}: 0.01-0.08)$ \\
Ever been hit by boyfriend & $.057^{* *}(95 \% \mathrm{CI}: 0.03-0.08)$ \\
Ever hit your boyfriend & $*$ Correlation is significant at the 0.05 level $(2$-tailed $)$ \\
\hline \multicolumn{2}{c}{$*$ Correlation is significant at the 0.01 level $(2-$ tailed $)$}
\end{tabular}

Risk behaviours predictive of teenage pregnancy

Logistic regression analysis (Table 4) showed significant associations between race and teenage pregnancy with being White having lower odds of ever been pregnant (OR: 0.374, 95\% CI: 0.203 - 0.691) than being Black African. Girls who engaged in first sexual intercourse at the age 13 years or younger had higher odds (OR: 1.299; 95\% CI: 0.812 2.078) of ever been pregnant than the girls who first had sex at the age 14 years or older. Having sex with 2 or more sexual partners had higher odds (OR: 1.250; 95\% CI: 1.039 1.503) of ever been pregnant than girls who had just one sexual partner. Condom use, as a contraceptive method used to prevent pregnancy had lower odds (OR: $0.462 ; 95 \%$ CI: $0.309-0.691$ ) of ever been pregnant compared to other methods of contraceptives used by the girls in this study. Girls using injection (OR: 1.142, 95\% CI: $0.747-1.746$ ) or birth control pills (OR: 1.004, 95\% CI: 0.618 - 1.762) for contraception had higher odds of ever been pregnant.

Among the smoking and substance use related behaviours; smoking cigarettes (OR: 0.641, 95\% CI: $0.497-0.825$ ), binge drinking (OR: 0.624, 95\% CI: $0.503-0.774$ ) and mandrax use (OR: 1.968, 95\% CI: 1.243 - 3.117) had higher odds as predictors of ever been pregnant during teenage years. Thus, adolescents smoking cigarettes and or using these drugs were more likely to ever been pregnant, than adolescent who were non-users. 
Table 4. Results of binary logistic regression analyses on ever been pregnant (dependent variable) with socio-demographics and risk behaviours (independent variables) from adolescent girls who ever had sex $(\mathrm{N}=5060)$

\begin{tabular}{|c|c|c|c|}
\hline \multirow[b]{2}{*}{ Variables } & \multicolumn{3}{|c|}{ Have you ever been pregnant } \\
\hline & $\mathbf{N}$ & OR & $95 \% \mathrm{CI}$ \\
\hline \multicolumn{4}{|l|}{ Demographic characteristics } \\
\hline \multicolumn{4}{|l|}{ Race } \\
\hline Black African (Reference) & 3261 & & \\
\hline Coloured & 408 & 1.047 & $0.762-1.439$ \\
\hline Indian & 30 & 0.649 & $0.219-1.917$ \\
\hline White & 201 & 0.374 & $0.203-0.691$ \\
\hline Other & 30 & 1.676 & $0.738-3.810$ \\
\hline \multicolumn{4}{|l|}{ Age (in years) } \\
\hline$\leq 13$ (Reference) & 58 & & \\
\hline 14 & 130 & 0.891 & $0.416-1.909$ \\
\hline 15 & 258 & 0.748 & $0.371-1.507$ \\
\hline 16 & 352 & 0.675 & $0.340-1.341$ \\
\hline 17 & 361 & 0.619 & $0.311-1.232$ \\
\hline$\geq 18$ & 2771 & 0.724 & $0.384-1.367$ \\
\hline \multicolumn{4}{|l|}{ Socio-economic status } \\
\hline \multicolumn{4}{|l|}{ Does your father have a paying job } \\
\hline Yes, works 5 or more days a week (Reference) & 1383 & & \\
\hline Yes, works less than 5 days a week & 292 & 0.907 & $0.641-1.283$ \\
\hline No, he gets a social grant & 479 & 1.019 & $0.768-1.352$ \\
\hline No, he is unemployed & 354 & 1.430 & $1.061-1.928$ \\
\hline No, he is ill or disabled & 113 & 1.483 & $0.922-2.384$ \\
\hline No, he has retired & 423 & 0.807 & $0.594-1.097$ \\
\hline My father is deceased & 498 & 1.451 & $1.112-1.893$ \\
\hline I don't know & 388 & 1.119 & $0.814-1.537$ \\
\hline \multicolumn{4}{|l|}{ Does your mom have a paying job } \\
\hline Yes, works 5 or more days a week (Reference) & 1200 & & \\
\hline Yes, works less than 5 days a week & 443 & 1.007 & $0.747-1.358$ \\
\hline No, she gets a social grant & 690 & 1.195 & $0.929-1.537$ \\
\hline No, she is a housewife & 914 & 0.962 & $0.757-1.222$ \\
\hline No, she is ill or disabled & 82 & 1.132 & $0.641-1.999$ \\
\hline No, she has retired & 90 & 1.218 & $0.711-2.089$ \\
\hline My mother is deceased & 289 & 1.134 & $0.811-1.586$ \\
\hline I don't know & 222 & 1.484 & $1.018-2.163$ \\
\hline \multicolumn{4}{|l|}{ Sexual activity and related behaviours } \\
\hline First sex at age $\leq 13$ & 267 & 1.299 & $0.812-2.078$ \\
\hline \multicolumn{4}{|l|}{ Number of sexual partners } \\
\hline 1 person (Reference) & 2599 & & \\
\hline 2 or more persons & 1331 & 1.250 & $1.039-1.503$ \\
\hline Ever use alcohol before sex & 407 & 1.373 & $1.004-1.878$ \\
\hline \multicolumn{4}{|l|}{ Condom use } \\
\hline Rarely (Reference) & 1195 & & \\
\hline Mostly & 2735 & 0.790 & $0.649-0.962$ \\
\hline \multicolumn{4}{|l|}{ Contraceptive methods } \\
\hline No method (Reference) & 570 & & \\
\hline Birth control pills & 160 & 1.004 & $0.618-1.762$ \\
\hline Condoms & 1697 & 0.462 & $0.309-0.691$ \\
\hline Injection & 557 & 1.142 & $0.747-1.746$ \\
\hline Other & 341 & 0.602 & $0.370-0.978$ \\
\hline \multicolumn{4}{|l|}{ Violence } \\
\hline Ever been hit by your boyfriend & 950 & 1.204 & $0.975-1.488$ \\
\hline
\end{tabular}




\begin{tabular}{|c|c|c|c|}
\hline Ever hit your boyfriend & 693 & 1.247 & $0.987-1.575$ \\
\hline Ever forced to have sex & 655 & 0.974 & $0.768-1.235$ \\
\hline Ever forced someone to have sex & 287 & 1.165 & $0.845-1.607$ \\
\hline \multicolumn{4}{|l|}{ Psychological well-being } \\
\hline Ever had serious sad and hopeless feelings & 1349 & 1.075 & $0.898-1.287$ \\
\hline Suicidal & 1086 & 0.938 & $0.808-1.196$ \\
\hline \multicolumn{4}{|c|}{ Smoking and substance use related behaviours } \\
\hline Smoked cigarettes & 869 & 0.641 & $0.497-0.825$ \\
\hline Alcohol use & 2451 & 0.871 & $0.718-1.057$ \\
\hline Binge drinking [s: & 1331 & 0.624 & $0.503-0.774$ \\
\hline Marijuana sis: & 466 & 1.315 & $0.970-1.782$ \\
\hline Sniffed glue, sspe & 329 & 0.979 & $0.711-1.348$ \\
\hline Mandrax & 135 & 1.968 & $1.243-3.117$ \\
\hline Cocaine & 123 & 1.549 & $0.935-2.566$ \\
\hline Heroin & 211 & 1.021 & $0.696-1.499$ \\
\hline Injection & 154 & 1.176 & $0.764-1.808$ \\
\hline Other & 150 & 0.839 & $0.510-1.380$ \\
\hline
\end{tabular}

*Bold means the variable is significant, $\mathrm{p}<0.05$

\section{Discussion}

The first important finding in this study is that sexual intercourse among adolescents has decreased. However, among those adolescent girls who reported to engage in sex, teenage pregnancy has increased. These findings are in line with increasing evidence demonstrating the magnitude of teenage pregnancy among sexually active adolescents, especially in the sub-Saharan African countries [Martin et al., 2012; WHO, 2015; Neal et al., 2015; Bhana et al., 2010; Mkwanazi et al., 2010]. This is worrying, particularly the fact that it suggest that there is little progress made in reducing teenage pregnancy over the past few decades, despite its importance as highlighted in the Millennium Development Goals (MDG) and its association to maternal mortality and morbidity rates [Ganchimeg et al., 2014]. Teenage pregnancy in developing countries has been reported to be worse than in developed countries [Neal et al., 2015; Ganchimeg et al., 2014]. The lack of data on teenage pregnancy trends among South African adolescents poses a serious public health threat, as the magnitude of the problem is relatively hidden for consideration into intervention programs that aim to reduce teenage pregnancy, and improve maternal and child health outcomes. The fluctuating rates of sexual intercourse at the age of 13 years or younger among girls is also concerning. Early sexual debut is not uncommon in SA. Recent research shows that more and more adolescents are sexually active by the time they are 14 years and older [Kirby et al., 2001; Mchunu et al., 2013]. 
This study shows that the trends in teenage pregnancy have remained steady from 2002 to 2011. When looking at those girls who reported to ever had sex, teenage pregnancy has even increased from 2002 (17.3\%) to 2011 (21.3\%). The increased pregnancy rates among these girls can be attributed to, among other factors, the fewer girls who are using contraceptive methods to prevent pregnancy but are sexually active in this study, despite the fact that contraceptives are available for free at the public healthcare settings in the country. Contraceptives, which are directly provided from the healthcare setting, such as birth control pills and contraceptive injections, are among the least of contraceptive methods used by adolescent girls in this study. The findings of small proportion of girls using modern contraceptives, such as birth control pills is surprising given the fact that contraceptives are provided free of charge in SA [Schnieder et al., 1999; Wilkinson et al., 2001; DoH, 2002]. However, this can be explained by (1) the poor knowledge of contraceptives among adolescents in general in SA [Kaufman et al., 2001; Mchunu et al., 2013], and (2) the limited availability of, and access to sexual and reproductive health services targeted at adolescents [Chandra'Mouli et al., 2013; Somba et al., 2014]. Furthermore, the cultural or societal, structural, and individual factors associated with the use of contraceptives are some of the barriers for contraceptive use [Somba et al., 2014; Watts et al., 2014]. These include, the need to prove fertility and maturity by the girls, opposition from male partners, perception of personal low risk around the ability to fall pregnant, and fear of side effects [Wood et al., 2006; Ochako et al., 2015; Watts et al., 2014; Obare et al., 2012; Hendriksen et al., 2007]. On one hand, low levels of contraceptive use and the attitude toward using them among adolescents has been associated with high rates of unwanted pregnancies and unsafe abortions among youth in Sub-Saharan Africa [Somba et al., 2014; Obare et al., 2012]. On the other hand, low levels of contraceptive use has also been attributed to limited capacity of the health care system, particularly in rural settings and structures within which family planning services are offered [Jewkes et al., 2009]. There is increasing evidence on the youth unfriendliness of the primary health care facilities in the country, which in turn might be a contributing factor to the poor use of contraceptives among adolescents; and thus exacerbate the trends in teenage pregnancy in SA [Hendriksen et al., 2007; Manzini et al., 2001; Shisana et al., 2014]. 
The second important finding in this study is that girls who use condoms when having sexual intercourse were less likely to get pregnant as a teenager. This finding supports previous research on reported knowledge and potential benefits of consistent and correct condom use by most young people in SA [Hendriksen et al., 2007; Manzini et al., 2001]. It is well known that condom use during sexual intercourse not only prevents unwanted pregnancy; but also prevents contraction of HIV and other STIs [Hendriksen et al., 2007; Manzini et al., 2001]. This finding also shows that the efforts made by the government in making condoms free of charge and easily accessible are beneficial to the youth of SA [Pettifor et al., 2005; Hendriksen et al., 2007; Shisana et al., 2014]. This is also in line with research findings from HICs where decline in teenage pregnancy is attributed to improved contraceptive use, including condom use [Santelli et al., 2007]. Therefore, continuous efforts on encouraging and facilitating condom use among young people in SA still proves to be an important behavioural intervention worth investing in, both for the prevention of HIV and other STIs' transmission and for contraceptive purposes.

Thirdly, girls who have sex with only one sexual partner in this study were also less likely to fall pregnant compared to the girls who had 2 or more sexual partners during their teenage years. Multiple sex partners is common in SA and has gender and cultural connotations, especially in KwaZulu Natal (KZN) and the Eastern Cape (EC) rural communities [Pettifor et al., 2005; Manzini et al., 2001; Shisana et al., 2014; Varga et al., 2003]. Having more than one sexual partner for boys in KZN, as well as in the EC proves "masculinity" among boys, while for some girls it guarantees peer approval although it has to be discreet among girls [Pettifor et al., 2005; Manzini et al., 2001; Varga et al., 2003; Jewkes et al., 2009; Jewkes et al., 2001]. This highlights another important area needing intervention for behaviour change, since a quarter of the girls in this study reported to have sex with 2 or more sexual partners.

The fourth and last finding of this study is the larger proportion of adolescents using alcohol and drugs. Clearly, alcohol and substance use among adolescents also requires attention. About half of the adolescents in this study have used alcohol one or more times in their lives. Substance use, in a few studies has been linked to teenage pregnancy. A study in Sweden reported that smoking and binge drinking among males was associated with the likelihood of the males having made their partners pregnant [Holmberg et al., 2002]. In this study, girls who never use alcohol before having sex, not practice binge 
drinking, who do not smoke cigarettes and do not use mandrax were less likely to fall pregnant as a teenager. The risk factors associated with teenage pregnancy, including alcohol use, smoking (tobacco or drug use) were also reported by [Imamura et al., 2007]. The author reported that these health risk behaviours tend to co-occur with teenage pregnancy in the European countries. Some studies have also reported on the possible link between teenage pregnancy and alcohol, and or tobacco and drug use [Imamura et al., 2007; Ebrahim et al., 1996; Silverman et al., 2001].

The findings of this particular study show that substance use increases the likelihood of adolescents falling pregnant during their teenage years. This is in line with previous findings that these risk behaviours, especially alcohol use, have been associated with other sexual-risk taking behaviours that result in pregnancy [Richter et al., 1996]. This study also found that girls who had a father who is unemployed or deceased were more like to get pregnant; and in terms of race, girls who belonged to the White race were less likely to become pregnant than other races. This finding supports previous research where low socio-economic status was found to be associated with teenage pregnancy in SA [Mkhwanazi et al., 2010; Cavazos-Rehg et al., 2011; Palen et al., 2006]. It is well documented in SA that teenage pregnancy is much more prevalent among the poor Black Africans [Mkhwanazi et al., 2010; Cavazos-Rehg et al., 2011].

A limitation of the study at hand is that it is undertaken in the school setting. This excludes those adolescents who were not schooling at the time of data collection. Teenage pregnancy has been reported to be the most common reason for school-dropout in SA [Cavazos-Rehg et al., 2011; Taylor et al., 2003]. Therefore, some adolescents might be out of school as a result of pregnancy and that does not invalidate the findings of this study, which confirms teenage pregnancy is still prevalent among adolescents in SA. Another limitation is that the results of this study cannot prove causality of teenage pregnancy by these risk behaviours, but only associations with teenage pregnancy. Thus, future research should aim to mitigate the risk behaviours associated with teenage pregnancy, as reported in this study. The pattern in teenage pregnancy is clearly increasing among those who engage in sexual activities, and that is a cause for concern. This suggests the further need to address health risk behaviours, including early sexual debut, unsafe sex, substance use and encouraging the correct and consistent use of contraceptives among adolescents; as well as promote their sexual and reproductive health in SA. 


\section{Conclusions}

Sexual intercourse among adolescents is decreasing, but teenage pregnancy continues to increase among those who are sexually active. Teenage pregnancy continues to be a growing public health concern and adolescent sexual and reproductive health behaviours, such as contraceptives and safe sex practices has to be acknowledged in the health care system as an important health issue in SA. Risky behaviours, such as substance use, also need to be recognized and intervention programmes aiming to reduce teenage pregnancy and sexual and reproductive behaviours among adolescents need to include prevention of substance use. This study highlights the need for more comprehensive research on adolescents SRH needs particularly among the vulnerable adolescents aged 16 years and under, but not excluding those aged above 16 years old, to identify risk factors and develop specific interventions tailored for their needs. Regular monitoring of teenage pregnancy trends and the associated factors also aid in determining the effectiveness of programmes put in place by government and other related institutions, as well as highlighting areas where extra effort is needed to curb the rates of teenage pregnancy. 


\title{
CHAPTER 3
}

\section{Published as}

Jonas K, Crutzen R, van den Borne B, Reddy P. Healthcare workers' behaviors and personal determinants associated with providing adequate sexual and reproductive healthcare services in sub-Saharan Africa: a systematic review.

\begin{abstract}
Background: Healthcare workers may affect the utilization of sexual and reproductive healthcare (SRH) services, and quality of care thereof, for example by their behaviours or attitudes they hold. This can become a hindrance to accessing and utilizing SRH services, particularly by young people, and thus a better understanding of these behaviours and associated factors is needed to improve access to and utilization of SRH services.

Methods: A systematic review of literature was conducted to identify studies focusing on healthcare workers' behaviors and personal determinants associated with providing adequate SRH services in sub-Saharan Africa (January 1990 - October 2015). Five databases were searched until 30th October 2015, using a search strategy that was adapted based on the technical requirements of each specific database. Articles were independently screened for eligibility by two researchers. Of the 125-screened full-text articles, 35 studies met all the inclusion criteria.

Results: Negative behaviours and attitudes of healthcare workers, as well as other personal determinants, such as poor knowledge and skills of SRH services, and related factors, like availability of essential drugs and equipment are associated with provision of inadequate SRH services. Some healthcare workers still have negative attitudes towards young people using contraceptives and are more likely to limit access to and utilization of SRH by adolescents especially. Knowledge of and implementation of specific SRH components are below optimum levels according to the WHO recommended guidelines. Conclusions: Healthcare workers' negative behaviours and attitudes are unlikely to encourage women in general to access and utilize SRH services, but more specifically young women. Knowledge of SRH services, including basic emergency obstetric care (EmOC) is insufficient among healthcare workers in SSA.
\end{abstract}

Protocol registration: A protocol for this systematic review was registered with PROSPERO and the registration number is: CRD42015017509. 


\section{Introduction}

Sexual and reproductive healthcare (SRH) and rights of women, including adolescents was placed on the international agenda in 1994, setting the tone for their priority in public health [Bearinger et al., 2007; Kuruvilla et al., 2016]. Following the millennium development goals (MDG), SRH services are now further prioritized in the sustainable development goals (SDG), more specifically SDG target 3.7, which aim to achieve universal access to sexual and reproductive healthcare services by 2030 [WHO, 2015]. However, SRH needs for many women, particularly adolescents are still not met despite these global agreements. SRH services remain grossly inadequate, especially in low- and middleincome countries (LMICs) [Kuruvilla et al., 2016]. This inadequacy is congruent with high rates of maternal mortality and morbidity, infant and child mortality, sexually transmitted infections (STIs), including HIV, unwanted pregnancies particularly among adolescents, and unsafe and illegal termination of pregnancy (TOP). Consequently, the burden of SRH related diseases and mortality is high in LMICs, especially in sub-Saharan Africa (SSA) [WHO, 2015; Hindin et al., 2009].

SSA still remains the region grossly affected by maternal deaths at 201000 in 2015 with more than $60 \%$ of all maternal deaths worldwide, being ascribed to the region [Hindin et al., 2009; WHO, 2012; World Bank Data, 2015]. SSA is amongst the regions affected by a high number of maternal mortality at 201000 in 2015 and was reported to have contributed to $62 \%$ of all maternal deaths worldwide in 2013 [Hindin et al., 2009; WHO, 2012; World Bank Data, 2015]. Maternal mortality rates in SSA are the worst in the world, with 640 deaths per 100,000 live births [WHO, 2012; World Bank Data, 2015]. Of course, a variety of factors play a role in the different countries of SSA, such as the living conditions, poverty, healthcare systems of each country, burden of other diseases like HIV and AIDS, and cultural and religious practices. Caution therefore needs to be exercised when interpreting the healthcare services and outcomes from the different countries and when planning interventions for the improvement of SRH service provision. High maternal and child mortality rates in SSA are thought to be primarily due to a lack of resources; and failures of the healthcare system, poor pre- and post- natal care attendance, unsafe TOPs, especially among adolescents. For example, only $25 \%$ of postpartum mothers and newborns in SSA receive postnatal care within 48 hours of birth [Yeji et al., 2015]. Women in SSA face a 1 in 39 risk of dying in childbirth; and over 800 
women die every day due to complications in childbirth and pregnancy [WHO, 2012]. Adolescents are at higher risk for maternal mortality, including infant and child mortality compared to older women due to many reasons, such as incomplete physiological development and, their SRH needs and rights still remaining non-prioritized in many SSA countries [WHO, 2012; UNPFA, 2013].

Teenage pregnancy in SSA is the highest in the world, with more than half of all births occurring in this region [WHO, 2012; UNPFA, 2013; Jonas et al., 2016]. The second worldwide leading cause of death among the 15-19 year old adolescent girls is complications during pregnancy and childbirth, with adolescent girls below the age of 16 years at an even higher risk for these complications, and consequently death and severe morbidity compared to women above the age of 20 years [WHO, 2012]. Given these alarming SRH outcomes in SSA, a quest to understand how healthcare workers provide SRH services and what factors are associated with adequate provision of the services was borne.

SRH services are often provided in the public health settings, although not always readily available in many resource-constrained settings like those of SSA, due to a number of environmental and socio-demographic factors. Such factors include lack of essential drugs and equipment, distance and long travel times to the facilities, shortage of healthcare workers and long waiting times at the facilities. Nurses and midwives are the healthcare professionals at the forefront in many public health facilities and are the most common category of healthcare workers women and adolescents consult for their SRH needs. When healthcare workers are available, they are often under-utilized especially by adolescents for various reasons, such as healthcare workers' negative behaviors and attitudes [Keeney et al., 2004; Biddlecom et al., 2007; Glasier et al., 2006; Chaibva et al., 2010].

Negative behaviours and attitudes of healthcare workers potentially affect access to and utilization of SRH services by women and adolescents especially, and the quality of care thereof. For example, studies in SSA indicate that healthcare workers' negative behaviours discourage women from seeking antenatal care, and young people from attending clinics or follow-up visits [Biddlecom et al., 2007; Glasier et al., 2006; Chaibva et al., 2010; Ngomane et al., 2012; Geary et al., 2014]. Basic emergency obstetric care 
(EmOC), a component of SRH services, may also be significantly under-utilized by young people, especially adolescents who become pregnant during their adolescent years [Chaibva et al., 2010; Ngomane et al., 2012; Geary et al., 2014]. Hence, an in-depth understanding of healthcare workers' behaviours and factors associated with their behaviours is needed in order to improve access to and utilization of SRH services at large, and by young people [Hindin et al., 2009; Biddlecom et al., 2007; Zielinski et al., 2001; Tilahun et al., 2012].

Lack of respect for women's opinion and preferences for birthing options for example, including adolescents' privacy and confidentiality, and the ill treatment by healthcare workers were some of the reported negative behaviours that discouraged women from giving birth at the healthcare facility, and sexually active adolescents from seeking SRH services [Biddlecom et al., 2007; Wood et al., 2006; Kaufman et al., 2001]. Adolescents also face difficulties obtaining contraceptives at public health facilities due to healthcare workers' negative attitudes associated with the general social stigma towards adolescent who seek contraceptive services in SSA [Biddlecom et al., 2007; Glasier et al., 2006; Kaufman et al., 2001; Singh et al., 2005].

The availability, accessibility, and utilization of SRH services may significantly alleviate the high rates of maternal mortality and morbidity, teenage pregnancy, STIs and HIV, unsafe TOPs, and infant and child mortality and morbidity in SSA. However, in order to accomplish these promising health outcomes in SRH services, healthcare workers need to adequately provide these services to women and adolescents in need, without any prejudice and limitations. Adequate provision of SRH services to young people particularly, encompasses amongst others, offering a youth-friendly environment, possessing a positive attitude towards the young people who use the services, being knowledgeable about their SRH issues and needs, and a willingness to serve them.

Therefore, the objective of this systematic review was to determine healthcare workers' behaviours and related personal determinants associated with providing adequate and quality SRH services to women and adolescents in sub-Saharan Africa. To achieve this objective, this review sought to answer the following research questions: 1) which healthcare workers' behaviours and personal determinants are associated with access to and utilization of SRH services in SSA? 2) How do these behaviours and personal 
determinants contribute to a good quality SRH services provided to women and adolescents in SSA? In this review, behaviours are defined as ways in which healthcare workers act and or conduct themselves towards clients seeking SRH services. Thus, healthcare workers' behaviours in this study encompass the manner in which a healthcare worker communicates with SRH clients, and any prejudice actions and tendencies unsupportive of SRH and rights of women and adolescents. Personal determinants refers to factors at the individual level, associated with the behaviours of healthcare workers in SRH services which may compromise the quality of the services, and that are potentially changeable by an intervention. Such factors include attitudes, basic knowledge, and skills regarding SRH services.

This systematic review to our knowledge, is the first review focusing especially on healthcare workers' behaviours and related personal determinants associated with provision of SRH services at large in SSA. It seeks to provide an overview of the behaviours, and related personal determinants of professional healthcare workers in SRH services, which potentially affect adequate provision of SRH services to women and young people. This overview is useful to identify healthcare workers' behaviours and related personal determinants needing improvement for adequate provision of SRH services. This in turn, will help develop specific interventions that aim to change the unwelcoming healthcare workers behaviours, and ultimately improve access to and utilization of SRH services by those in need, especially young people.

\section{Methods}

To answer the above research questions, a systematic review was undertaken. A protocol, registered with PROSPERO (registration number: CRD42015017509) in advance [Jonas et al., 2015], was developed as a guide to direct the review processes, and can be accessed here:

http://www.crd.york.ac.uk/PROSPERO/display record.asp?ID=CRD42015017509

\section{Search strategy}

The following electronic databases were searched (from January 1990 until October October 2015): PubMed, EMBASE (through OVID), Cochrane central (through Cochrane library), CINAHL (through EBSCOhost), and PsychINFO (through EBSCOhost) using a 
search strategy that was developed based on the key words identified from the study objective, which was adapted according to the technical requirements of each specific database. The full search strategy is provided as Additional file 1.

The search period included studies published since January 1990 until October 2015. This period was chosen because of the MDG 2015 (target 5) pertaining to maternal and child health; which includes amongst others, efforts to reduce maternal mortality, teenage pregnancy, and unmet needs of SRH (family planning) services. Therefore, this review sought to identify studies which were either addressing, or attempting to, or were related to the means and efforts aiming to attain the MDG 5 targets in SRH services. The studies were restricted to: peer-reviewed and academic journals using the specific database filters available and by manual assessment to limit studies to primary studies only (e.g., no editorials, or reviews), studies undertaken in the sub-Saharan African region only, and studies that were published in English language.

\section{Eligibility criteria}

For studies to be included in this review, studies had to pass the eligibility criteria for inclusion: studies with participants as professional healthcare workers, including doctors, nurses, midwives, obstetricians and gyneacologists (Population); if the studies were focusing on behavioral and personal determinants of professional healthcare workers, such as education, knowledge and skills of professional healthcare workers in SRH services, studies involving contraceptives, abortion (TOP), pregnancy, childbirth; studies investigating factors associated with access to, utilization of, and quality of SRH services and related services (Intervention); if the primary outcomes were quality of healthcare in SRH services, access to SRH healthcare services, utilization of SRH services, and or the secondary outcomes were adolescent pregnancy, maternal health outcomes, and child health outcomes (Outcome); if study participants were working in the SRH services, including gyneacology and obstetric care (GOC) services, as well as maternal and obstetric units (MOU); and the studies involving services rendered under general public health facilities, such as primary healthcare clinics (PHC), community health centers (CHC), secondary and tertiary hospitals, as well as academic hospitals (Setting). Any types of study designs were eligible for inclusion provided that they reported quantitative data. Only studies with quantitative data focusing on either one or more of the primary and secondary outcomes were eligible. The rationale for the inclusion criteria of only 
quantitative studies was because this review sought to quantify the relationship between behaviours, determinants, and primary or secondary outcomes in order to inform future intervention development in terms of relevance of specific determinants. Because this review is focusing on determinants rather than comparing interventions or treatment, it does not have a comparator (No Control). The full PRISMA checklist is provided as Additional file 2. Two independent reviewers (KJ and RC) performed the selection process of the eligible studies. Studies which do not meet the inclusion criteria according to the independent reviewers were brought forward for discussion and reaching consensus regarding the study's eligibility for inclusion in this review.

\section{Review procedure}

The search was conducted in a step-wise manner with three phases. The first phase was a broad search that was developed with the guidance of an experienced librarian. The second phase involved searching of specific electronic databases based on the results of the first phase using the provided search terms that were revised and adapted from the first phase. The third and last phase was conducted for additional sources of data. This involved a manual search of reference lists of included reports and articles, and other online resources. Search results were imported into the Endnote database and duplicates were screened for and deleted. Articles went through an extensive screening process in order to identify sources of relevant data.

Initially, titles were scanned for relevance and titles obviously not meeting the inclusion criteria were excluded. Then, the screening of abstracts of those titles that met the inclusion criteria began, and abstracts not meeting the inclusion criteria were excluded. Following the screening of abstracts was the reading of full-text articles of the included abstracts, and articles not meeting all the inclusion criteria were excluded, as well as the articles that did not have full text available. The reasons for exclusion of the full-text articles were either: the article did not report on the behaviours of healthcare workers, or the article reported behaviours based on qualitative findings, or the article reported behaviours of non-professional healthcare workers such as community healthcare workers. Finally, the rigorous and extensive assessment of the included full-text articles as they met the inclusion criteria was conducted. Again, the two independent reviewers (KJ and $\mathrm{RC}$ ) performed the process described above and met regularly to discuss their assessment until consensus was reached. 
Experts in the field of maternal and child healthcare, obstetric and gynaecology were also contacted and asked to recommend any new data sources not yet identified through the means described above, including unpublished data. Of the twelve experts contacted via email, only two responded with sources of information consisting of articles not available through the databases searched. The flow diagram below shows the review process from the initial searches of bibliographic databases to the final included studies (see Figure 1.). 


\section{Review process}

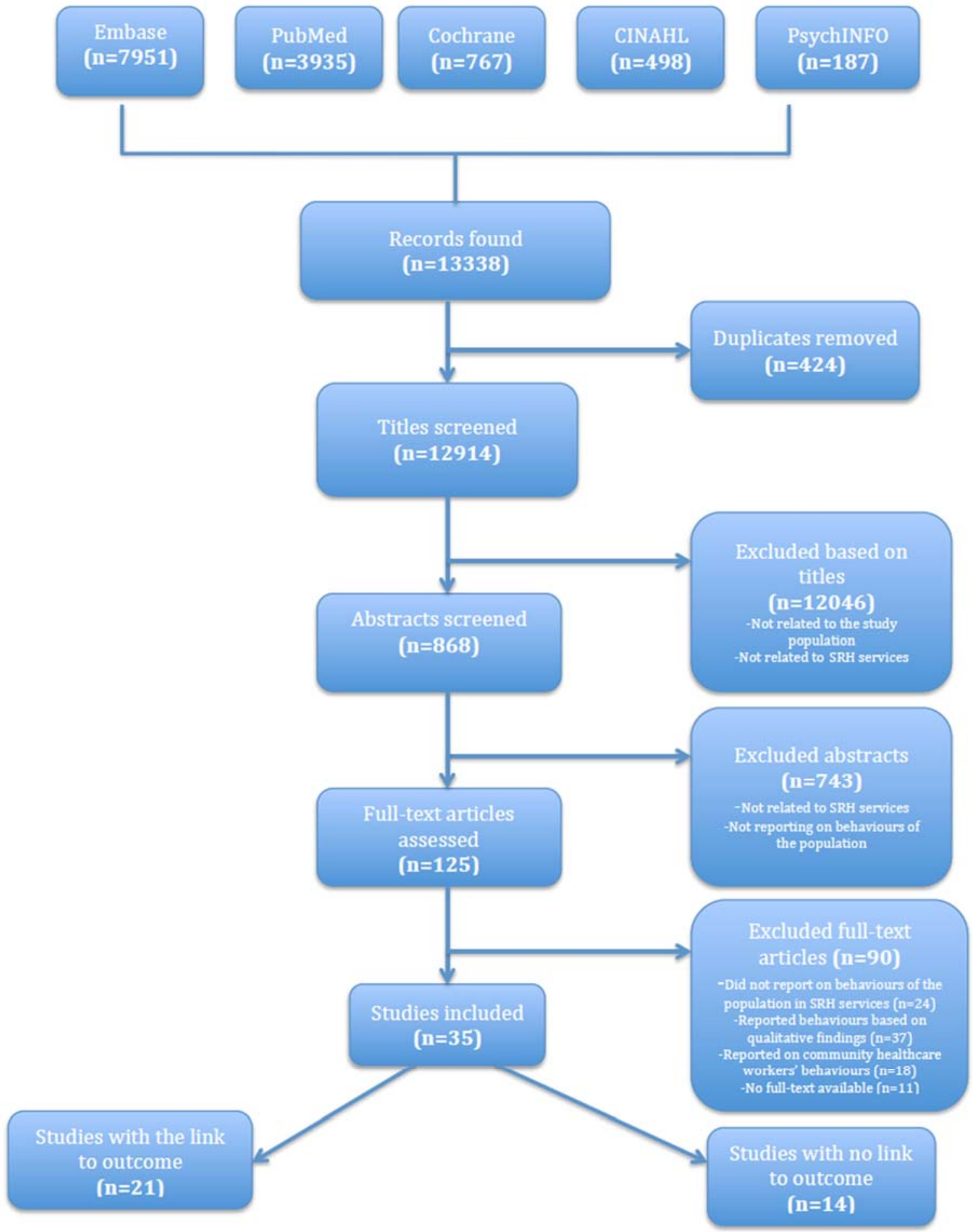

Fig. 1 Flow Diagram (PRISMA) showing the different stages of the review process from the initial searches of bibliographic databases that yielded 13338 records to the final included studies.

\section{Data abstraction}

Publication year, authors, location of study, research design, study participants and outcome measure, as well as data regarding determinants were key factors for data 
extraction. Data abstraction was carried out by the two independent reviewers (KJ and RC) and they met regularly to discuss their assessment and address any discrepancies until consensus was reached. Where consensus was not reached for a study, the particular study was taken to the next step for further screening and assessment until both reviewers were in consensus. This was also done in order to strengthen the quality of the analysis of the studies included, as well as not immediately excluding studies based on one reviewer's assessment while studies could potentially be eligible when thoroughly assessed by more than one reviewer. Data from these studies included was extracted and tabulated in Microsoft Excel. Quality assessment of the studies included was assessed using the STROBE checklist for observational studies tool which covers data collection, methods, results, discussion and other information relevant for this review such as the study population. Quantitative data collection methods (e.g. surveys), type of study design (e.g. cross-sectional designs), and description of study participants (e.g. nurses) were among the key criteria in the quality assessment of the studies.

\section{Data analysis}

A narrative analysis approach was used to synthesize the data. For all studies included in this review, data was extracted into a table eliciting information on: (1) first author and year of publication; (2) study sample and population; (3) study design; (4) study outcome; and (5) the determinant(s) with summary measures of the study findings (e.g., attitudes towards SRH services, knowledge of specific SRH components, use of specific SRH resources, and obstetric skills) (see Table 1). Furthermore, studies were assessed and data was synthesized for potential to be associated with or impact on the primary or secondary outcomes (see Table 2).

\section{Results}

A total of 13,338 titles were identified through the five electronic databases searched. After removal of duplicates $(n=424), 12914$ title records were screened and titles not meeting the inclusion criteria were excluded. Screening of the identified titles resulted in 868 abstracts that were reviewed. A total of 125 full articles were then examined for relevance to the review and 35 studies met all the inclusion criteria and therefore are included in this review (see Table 1). 
As shown in the flow diagram of the review process (see Figure 1.), of the ninety (90) articles excluded, 11 articles were not full-text available, 24 articles did not provide any information on behaviours and attitudes of healthcare workers, 18 articles reported behaviours of non-professional healthcare workers, and the remaining 37 articles reported qualitative findings of behaviours and patients or clients views of healthcare workers' behaviours and attitudes in SRH services. 
Table 1. Main table with all included studies $(n=35)$

\begin{tabular}{|c|c|c|c|c|}
\hline Study/Country & Sample & Design & Outcome & Determinant \\
\hline $\begin{array}{l}\text { Tilahun et al. } \\
\text { (2012)/Ethiopia }\end{array}$ & $\begin{array}{l}\text { Physician and } \\
\text { nonphysician } \\
\text { obstetric care } \\
\text { workers }(n=423)\end{array}$ & $\begin{array}{l}\text { Cross-sectional } \\
\text { study }\end{array}$ & $\begin{array}{l}\text { Attitude toward provision } \\
\text { of SRH to unmarried } \\
\text { adolescents }\end{array}$ & $\begin{array}{l}\text { Negative attitude toward provision of SRH services to } \\
\text { adolescents was significantly associated with healthcare } \\
\text { workers' being married (OR: } 2.15 ; 95 \% \text { CI } 1.44-3.06 \text { ), lower } \\
\text { levels of education (OR: } 1.45 ; 95 \% \text { CI } 1.04-1.99 \text { ), being a } \\
\text { health extension worker (OR: } 2.49 ; 95 \% \text { CI } 1.43-4.35 \text { ), lack } \\
\text { of training on RH services (OR: } 5.27 ; 95 \% \text { CI } 1.51-5.89 \text { ), and } \\
\text { non-use of family planning (OR: } 1.77 ; 95 \% \text { CI } 1.05-2.77 \text { ). }\end{array}$ \\
\hline
\end{tabular}

\section{Okokon et al. \\ (2014)/Nigeria.}

Fawole et al. (2008)/

Nigeria

Fawole et al. (2010)/

Nigeria
Nonphysician

obstetric care

workers $(n=290)$

Physician and

nonphysician

obstetric care

workers $(n=719)$
Cross-sectional

study

Cross-sectional

study

Utilization of the

partograph

Knowledge and utilization of the partograph

Knowledge of the partograph had a significant relationship with its utilization in teaching hospital $\left(\chi^{2}=38.96, \mathrm{p} \leq 0.0001\right)$ and general hospital $\left(\chi^{2}=12.05, \mathrm{p} \leq 0.0001\right)$, but not in primary healthcare centers $\left(\chi^{2}=0.08, \mathrm{p}=0.778\right)$

Utilization of partograph was reported more frequent by responded working in tertiary levels of care compared to those on primary and secondary levels $\left(\chi^{2}=214.6, p \leq\right.$ 0.0001). $37.3 \%$ of respondents who were predominantly from the tertiary level of care could correctly mention at least one component of the partograph $\left(\chi^{2}=139.1, p<0.0001\right)$. The partograph is utilized mainly in tertiary health facilities; knowledge about the partograph is poor.

Knowledge of partograph components was poor among auxillary nurse/midwives at $0 \%$ median score. Knowledge about assessment that could be made by the use of partograph was poor on both settings (private and primary/public care facilities). More responded in the private sector correctly mention the function of the alert line on the partograph than those on the public sector $\left(\chi^{2}=4.43, p<\right.$ $0.05)$. Previous training on partograph was associated with knowing at least one component of the partograph $\left(\chi^{2}=49.2\right.$, $\mathrm{p}<0.05)$. Knowledge about assessment of women during labour was also poor.

Knowledge about partograph was significantly higher among 
Wakgari et al. (2015)/ Ethiopia

Physician and nonphysician obstetric care workers $(n=403)$

\section{Opiah et al. (2012)/}

Nigeria

Hussein, et al. (2004)/ Ghana
Nonphysician obstetric care workers ( $\mathrm{n}=165)$

Physician and nonphysician obstetric care workers $(n=416$ deliveries)
Cross-sectional study

Descriptive crosssectional study

Knowledge and utilization of the partograph

Retrospective Obstetric skills study

Descriptive crosssectional study

Utilization of partograph
$40.2 \%$ utilized partograph during labor. Those who were midwives by profession were about 8 times more likely to have a consistent utilization of the partograph than general practitioners $(\mathrm{AOR}=8.13,95 \% \mathrm{CI}: 2.67,24.78)$. Similarly, getting on job training (AOR $=2.86,95 \% \mathrm{CI}: 1.69,4.86)$, being knowledgeable on partograph (AOR = 3. 79, 95\% CI: 2.05, 7.03) and having favorable attitude towards partograph (AOR = 2. 35, 95\% CI: 1.14, 4.87) were positively associated with partograph utilization.

Knowledge of partograph was significantly associated with is utilization $\left(\chi^{2}=32.29, \mathrm{p}<0.05\right)$, and between the obstetric care workers' years of experience and partograph utilization $\left(\chi^{2}=4.82, \mathrm{p}<0.05\right)$

For all deliveries, $32.6 \%$ to $93.0 \%$ of criteria were met for "standard care" (defined by Section A criteria), with a mean of $65.5 \%$. No delivery met all of the criteria. Doctors, with a mean score of $76.1 \%$, appear to satisfy more criteria than mid- wives at $67.4 \%$, who in turn satisfy more criteria than assistant midwives, a non-professional grade of staff in private facilities in Ghana.

A minority of the obstetric care workers routinely performed basic ANC services. EmOC services provided, including the use 


\section{Mirkuzie et al.}

(2014)/Ethiopia

Ameh et al. (2012)/

Somalia

Haile-Mariam et al

(2012)/Ethiopia
Nonphysician

obstetric care

workers $(n=49)$

Physician and

nonphysician

obstetric care

workers $(n=222)$

Nonphysician

obstetric care

workers $(n=711)$
Vivio et al. (2010)/ Zambia Nonphysician obstetric care
Health facility-

based interventio

Descriptive study

Cross-sectional

study

Observational cross-sectional study workers $(n=62)$

Knowledge of the active management of the third stage labour (AMTSL) provision of AMTSL

Observational study workers $(n=21)$

Nonphysician obstetric care workers $(\mathrm{n}=1561)$ study
Cross-sectional
Knowledge and skills (use) of maternal ultrasound Clinical decision-making

Intention to leave obstetric services (job satisfaction)
Knowledge and skills of basic EmOC

Knowledge and skills of life-saving EmOC, provision of EmOC Availability and quality of EmOC

Knowledge of EmOC, resuscitation

Zambia

McAuliffe et al. (2013)/ Malawi, Tanzania, Mozambique
Obstetric care workers' knowledge on basic Em0C was poor both in 2008 respondents as well as in 2013 respondents.

There was a significant improvement in knowledge (50\%) and skills (100\%) among the obstetric care workers. Confidence in EmOC provision was improved

Half of the midwives interviewed reported having performed neonatal resuscitation in the past three months compared to only $20 \%$ of the nurses. Key predictors of a high knowledge score among providers were recent performance of neonatal resuscitation and geographic region. Whether the provider was a nurse or a midwife, was not associated with a higher knowledge score.

Majority of the obstetric care workers knew that AMTSL was associated with the administration of a uterotonic drug. More than half of the participants were aware of the controlled cord tracting (CCT) as a strategy to promote placenta delivery, while a few reported early cord clamping and cutting as a component of the AMTSL protocol, and were observed to use it as a standard practice. A third of the responded were aware of the fundal massage as a component of the AMTSL protocol.

Paired OSCE scores showed a slight overall improvement in the midwives ability to scan at 2 months of $10.0 / 14$, SD 3.9 $(71 \%)$ and at 6 months of $11.6 / 14$, SD 1.8 (83\%). Paired t-test showed no significant difference between OSCE 1 and OSCE II with $\mathrm{p}=0.15$

Not receiving any supervision appeared to be most strongly linked to decrements in intentions to leave and job satisfaction. In particular, intentions to leave were substantially increased when no supervision system was in place in Malawi $(\mathrm{b}=1.09, \mathrm{SE}=0.31, \mathrm{t}=3.52, \mathrm{p}<0.01)$, 
Maramagi et al. (2004)/

Uganda

Tita et al. (2006)/

Cameroon

\section{Ijadunola et al. (2010)/}

Nigeria

Ersdal et al. (2008)/

Zimbabwe
Nonphysician

obstetric care

workers $(n=37)$

Physician and

nonphysician

obstetric care

workers $(n=328)$
Cross-sectional

study

Cross-sectional study
Counseling skills in IMCI programme

Awareness of evidencebased obstetric care

Tanzania $(\mathrm{b}=0.82, \mathrm{SE}=0.3, \mathrm{t}=2.73, \mathrm{p}<0.01)$, and Mozambique $(\mathrm{b}=1.04, \mathrm{SE}=0.39, \mathrm{t}=2.67, \mathrm{p}<0.01)$. There was also clear evidence of a link between the absence of supervision and diminished job satisfaction in all countries. In all three countries we found robust evidence indicating that a formal supervision process predicted high levels of job satisfaction and low intentions to leave.

Older health providers (50-59 years) were more likely to advise caregivers on medication than younger health providers $\left(\chi^{2}=15.64, \mathrm{p}=0.016\right)$. Male health providers $\left(\chi^{2}=\right.$ $6.22, \mathrm{p}=0.045)$ and those aged $30-39$ years $\left(\chi^{2}=19.244, \mathrm{p}=\right.$ $0.004)$ were more likely to explain the feeding problem to the caregivers. Health providers aged 30 or more years were more likely to give feeding advice than younger health providers $\left(\chi^{2}=9.62, \mathrm{p}=0.022\right)$.

A total of $15.5 \%(50 / 322)$ of health workers were aware of all the four interventions while only $3.8 \%$ (12/312) reported optimal practice. Evidence-based awareness was strongly associated with practice (OR $=15.4 ; 96 \%$ CI: 4.3-55.0). Factors significantly associated with awareness were: attending continuing education, access to the WHO RHL, employment as an obstetrician/ gynaecologist and working in autonomous military or National Insurance Fund facilities. Controlling for potential confounding, working as an obstetrician was associated with increased awareness (adjusted prevalence odds ratio [aPOR] $=8.3$; 95\% CI: $1.3-$ 53.8) as was median work experience of 5-15 years $(\mathrm{aPOR}=$ 2.0; 95\% CI: 1.0-3.8). Internet access was associated with increased practice (aPOR $=3.4 ; 95 \% \mathrm{CI}: 1.0-11.8$ ).

Knowledge of EmOC was poor among the respondents, and no difference across age groups. Doctors had a fair knowledge compared to other profession.

obstetric care

workers $(n=152)$

Physician and

nonphysician

obstetric care

Cross-sectional

study
Knowledge, attitude and practice of symphysiotomy
Seventy-nine of the 80 participants knew about symphysiotomy, and 76 could describe the technique, including 16 of the 17 midwife instructors. One junior doctor 
obstetricians had occasionally performed a symphysiotomy the other nine did not practice the intervention, but indicated that they would be able to carry it out. All the rural midwives $(\mathrm{n}=13)$ regarded symphysiotomy as a lifesaving operation appropriate for remote areas, and 23 of the 39 midwives (59\%) thought that the procedure should be taught to midwives.

Ndikom \& Onibokun (2007)/ Nigeria

Chi et al. (2004)/ Zambia

Physician and nonphysician obstetric care workers $(n=225)$
Nonphysician obstetric care workers $(n=155)$
Byamugisha et al. (2007)/ Uganda
Cross-sectional study

Cross-sectional study...

Perceptions toward HIV screening and treatment

nowledge and behaviour towards PMTCT
Physician and nonphysician obstetric care

workers $(n=247)$
Cross-sectional study
Knowledge, attitude and practice of EC
Nurse/midwives had moderate level of knowledge with mean score of $51.4 \%$. Hypotheses tested revealed that there is a positive relationship between knowledge and behaviour $(\mathrm{r}=$ $0.583, p=0.00$ ). Knowledge level of nurse/ midwives who had educational exposure was not different from those who did $\operatorname{not}(\mathrm{t}=1.439, \mathrm{p}=0.152)$. There was a significant difference in the knowledge of nurse/midwives who had experience in managing pregnant women living with HIV/AIDS and those who did not $(\mathrm{t}=2.142, \mathrm{p}=0.03)$. Also, there was a significant relationship between behaviour and availability of resources $(\mathrm{r}=0.318, \mathrm{p}=0.000)$.

Providers reported widespread stigma associated with HIV. Physicians $(\mathrm{OR}=1.9)$, providers with research affiliations (OR $=2.3$ ), and those located in Lusaka (OR $=9.0$ ) were more likely to offer HIV testing. Only 30\% routinely prescribed antiretroviral treatment (ART) to reduce MTCT. Practitioners from district facilities, and those employed at research facilities were more likely to prescribe ART routinely $(\mathrm{OR}=$ 2.8, 10.1 and 3.4 respectively). Among those never prescribing ART, most cited a lack of availability (83\%).

Most of the participants had heard about EC (79.4\%). 1 in 4 $(24.1 \%)$ of the participants did not know the time limit within which EC is effective. slightly more than half (53.3\%) wanted to make the population sensitized about EC issues. About $28 \%$ wanted EC available and accessible in convenient places for all. Some HCWs expressed the need for more training, such as seminars in FP methods. Almost half (49\%) of the participants who knew about EC had prescribed it in one form or another 
and 1 in every 10 had prescribed ECPs in the previous 12 months $(11.9 \%)$

Traore et al. (2014)/ Mali Physician and nonphysician obstetric care workers $(n=196)$

Ehiri et al. (2005)/Nigeria Nonphysician obstetric care workers $(n=252)$

Worku et al.

(2013)/Ethiopia

Nonphysician obstetric care workers $(n=38)$

Mngadi et al. (2008)/ Swaziland

Physician and nonphysician obstetric care workers $(n=56)$
Cross-sectional study

Knowledge and skills of EmOC

Cross-sectional descriptive study

\section{Quality of child health} services

Population-based survey

Exploratory study

Attitude towards adolescents' SRH service provision
Bivariate analysis showed an association between competency score and type of health worker ( $p<0.05$ ). Knowledge was most deficient for postpartum infection and hypertensive complications. Type of health worker, years of experience, number of days absent, and avail- ability of guidelines for management of obstetric complications within the health center were positively associated with test score (p $<0.05$ ). Availability of guidelines was associated with higher competency of physicians, health technicians, and obstetric nurses ( $p<0.001$ ), and seemed to influence the competency of healthcare workers with fewer than 10 years of experience in particular.

Facilities were adequately equipped with immunization supplies. Essential drugs supply was inadequate in all centers; as well as emergency care facilities. $68.3 \%$ of the respondents had adequate training in immunization with high knowledge scores on immunization issues. Use of the national case management algorithm was low among the respondents.

Availability of essential drugs, equipment and other supplies was unsatisfactory. A small proportion of obstetric care workers had adequate training and experience on important obstetric procedures. Only $24 \%$ of the obstetric care workers used partograph frequently and consistently. Majority of the facilities did not function fully for emergency obstetric care (EmOC) as per their level. Important ANC components were incomplete and unsatisfactory.

$78 \%$ respondents reported that they provided contraceptives to the adolescents when they asked for them and when they were available. Some reported that contraceptive use was against their religion and that youth should not indulge in sex. Half of the nurses/midwives had no continued education and lacked supervision on adolescent sexual and reproductive health care. The majority had unresolved moral doubts, 
Oduro-Mensah et al. (2013)/ Ghana

Leon, Lundgren, \& Jennings (2006)/ Rwanda

Nonphysician obstetric care workers $(n=40)$
Cross-sectional descriptive study obstetric care workers $(n=65)$

negative attitudes, values and ethical dilemmas towards abortion care between the law, which is against abortion, and the reality of the adolescents' situation. Forty-four wanted to be trained on post-abortion care while eight on how to perform abortions. Twenty-six wanted the government to support adolescent-friendly services and to train healthcare providers in adolescent sexual and reproductive health services.

Printed protocols and guidelines were the most commonly selected (96\%) aids used in decision-making. However workshop materials (92\%), expert advice (90\%) and telephone calls for advice (85\%) were also frequently selected as aids in daily decision making. The majority of respondents (80\%) said they had access to various local (institutionally) modified or developed guidelines and protocols e.g. management of postpartum haemorrhage, management of pre-eclampsia / eclampsia, etc. Health system constraints such as availability of staff, essential medicines, supplies and equipment; management issues (including leadership and interpersonal relations among staff), and barriers to referral were important influences in decision-making.

Observational study
Guidelines utilization for Provision of contraceptives

Providers implemented less than one third of the guideline set, but they addressed, more frequently than other guidelines, items categorized as essential by expert opinion ( $p$ $<$ ?0.01). Rwandan providers emphasized contraindications in 29-minute sessions. Within this set, items previously classified as essential were addressed more frequently than guidelines classified as less important in Rwanda (3:2). Use instructions were not addressed frequently than other guideline categories in Rwanda, where contraindications were more prevalent than use instructions. Contraindications ranked above use instructions in both locations in Rwanda.

nurse-midwives disapproved of adolescent sexual activity, including masturbation, contraceptive use and abortion, but also had a pragmatic attitude to handling these issues. Those with more education and those who had received continuing

Warenius et al. (2006)/ Kenya/Zambia
Nonphysician

obstetric care

workers $(n=707)$
Cross-sectional

study
Attitude towards adolescents' SRH service provision 
Evens et al. (2014)/ Kenya Nonphysician

\section{Chalmers, McIntyre \&} Meyer, (1992)/ SA

Sidze et al. (2014)/Senegal Physician and

Chaibva et al. (2010)/ Zimbabwe obstetric care workers $(n=20)$ nonphysician obstetric care workers $(\mathrm{n}=637)$ obstetric care

workers $(n=52)$
Descriptive postintervention study

Physician obstetric Hospital-based care workers $(n=203)$

education on adolescent sexuality and reproduction showed a tendency towards more youth- friendly attitudes. About twothirds (69\%) of Kenyan and about half (52\%) of Zambian respondents disagreed that "16-year-old out-of-school girls should be encouraged to use condoms". However, both Kenyan (55\%) and Zambian (67\%) nurse-midwives agreed that "if a schoolgirl was sexually active she should be allowed to use contraceptives".

Majority of healthcare providers' were in favour of equal treatment for PAC regardless of age/marital status of clients. All healthcare providers deemed PAC services important. Sexual activity after marriage was supported by $45 \%$ of the providers.

Attitude toward caesarean Private doctors consider CS safer than a "normal" delivery. section

Public doctors believe that CS are performed by obstetricians duet $o$ lack of proper (training) management of complication/difficult delivery.

Health facility Access to and use of survey

Descriptive study contraceptives

Utilization of prenatal services by adolescents
Mane et al. (2014)/Senegal
Physician and nonphysician
Descriptive study Provision of emergency contraceptives (EC)
Obstetric care workers had a minimum age restriction, as well as restrictions according to clients' marital status, for the provision of contraceptives to young women in Senegal.

Socio-demographic and cultural factors influenced adolescents' utilization of prenatal services according to the midwives. Almost all of the midwives (n $1 / 4$ 49; 94.2\%) considered financial constraints to be a factor limiting adolescents' utilization of prenatal services. $71.2 \%$ agreed that health workers' attitudes could influence adolescents' decisions. Most midwives (n $1 / 445 ; 86.5 \%$ ) reported that the quality of prenatal services could influence adolescents' decisions to utilize prenatal services; 29 (55.8\%) felt that adolescents perceived prenatal care to be beneficial because they realised that obstetric problems could be detected and addressed during the prenatal period.

Knowledge gaps about EC among obstetric care workers were found. They also reported reluctance in providing counseling 
obstetric care

workers $(n=163)$

Lawani et al. (2014)/

Nigeria

Physician obstetric

care workers

$(n=151)$
Cross-sectional

study
Provision of obstetric

analgesia

and consequently the service/availability of the service

thereof. The workers also carried judgment towards EC users.

A larger proportion of the obstetric care workers indicated

unwillingness to provide EC to adolescents- only $37 \%$

indicated they would provide EC to adolescents who require it.

A total of 74 (49.0\%) participants offered obstetric analgesia to parturients in labour that were either non- assisted or assisted with instruments. Among users, only 20 (13.3\%) offered obstetric analgesia routinely to parturients, 44 $(29.1 \%)$ sometimes and $10(6.6 \%)$ on patients' requests. The comparison between the younger and older age groups was statistically significant with Odds Ratio of 4.37 (1.18-16.18) at $95 \%$ CI, $p=0.018$, while that between urban and rural practitioners was $4.77(0.99-22.85)$ at $95 \% \mathrm{CI}, \mathrm{p}=0.034$. 


\section{Included studies}

A majority of the studies were from Nigeria $(n=9)$ and Ethiopia $(n=7)$, followed by Kenya $(\mathrm{n}=2)$, Uganda $(\mathrm{n}=2)$, Zambia $(\mathrm{n}=2)$, Senegal $(\mathrm{n}=2)$, Ghana $(\mathrm{n}=2)$, Zimbabwe $(\mathrm{n}=2)$, and South Africa $(\mathrm{n}=2)$. Malawi, Swaziland, Cameroon, and Rwanda were represented by one study each, and one study represented Mali, Tanzania and Mozambique in one. Of the 35 studies included (see Figure 1), 21 of them investigated association or influence on the primary outcomes, which affect quality of SRH services and possibly access to and utilization of the services, as described above (see Table 2). Fourteen studies did not investigate this association and only reported on healthcare workers' behaviours and related factors in SRH services in a descriptive sense. 
Table 2. Studies associated with the primary or secondary outcomes

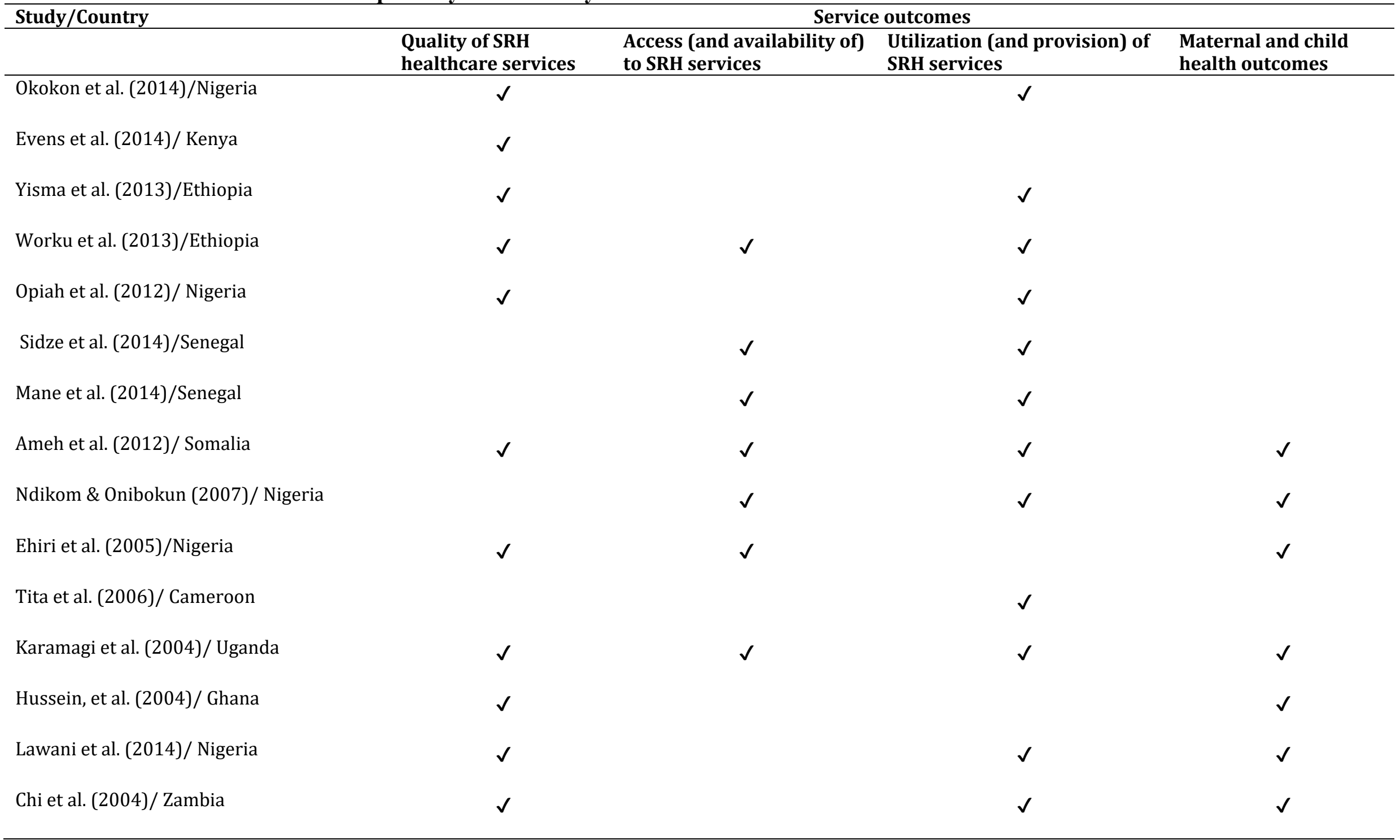




\begin{tabular}{|c|c|c|c|}
\hline Wakgari et al. (2015)/ Ethiopia & $\checkmark$ & $\checkmark$ & $\checkmark$ \\
\hline Nyango et al. (2010)/Nigeria & $\checkmark$ & & $\checkmark$ \\
\hline Vivio et al. (2010)/ Zambia & & & $\checkmark$ \\
\hline Chaibva et al. (2010)/Zimbabwe & & $\checkmark$ & $\checkmark$ \\
\hline
\end{tabular}


Of the 35 studies included, 28\% ( $\mathrm{n}=10)$ of them investigated basic obstetric care skills among professional healthcare workers, $23 \%(n=8)$ investigated knowledge of SRH service components, $20 \%(n=7)$ investigated behaviours of professional healthcare workers with regards to certain SRH services. The remaining 29\% $(n=10)$ studies investigated availability of resources and other essential components of SRH services, as factors associated with adequate quality service provision in SRH services. A majority of the studies, $71 \%(n=25)$ were cross-sectional studies, some were population and hospital surveys $9 \%(n=3)$, exploratory studies $6 \%(n=2)$, observational studies 6\% $(n=2)$, intervention studies $6 \%(n=2)$, and only one was a retrospective study $3 \%(n=1)$.

The included studies investigated determinants associated with adequate, quality service provision in sexual and reproductive healthcare (SRH) services and other related factors. The determinants included basic knowledge and skills regarding SRH among professional healthcare workers working in SRH services, as well as their behaviours and attitudes towards providing SRH services. Other factors associated with the behaviours and personal determinants of healthcare workers in SRH, such as the availability of essential SRH resources are also reported. The results are presented under the research question which this review sought to answer.

Which healthcare workers' behaviours and personal determinants are associated with access to and utilization of SRH services in SSA?

In this paper, a description of healthcare workers' behaviours and personal determinants associated with these behaviours are reported. These behaviours and personal determinants have potential to negatively affect SRH access and utilization of services, as well as quality of care.

\section{Knowledge and skills of obstetric care in SRH services}

Among the studies investigating knowledge, eight of them focused on knowledge of partograph $(n=8)$, a component of basic emergency obstetric care (EmOC), and its association with its utilization in SRH services. These studies were undertaken in Nigeria ( $n=5)$, Ethiopia ( $n=2)$, and Ghana $(n=1)$ [Okokon et al., 2014; Fawole et al., 2008; Fawole et al., 2010; Yisma et al., 2013; Wakgari et al., 2015; Opiah et al., 2012; Hussein, et al., 2004; Nyango et al., 2010]. All these studies found that knowledge of partograph 
had a strong association with its utilization in SRH services. Knowledge of partograph was found to be higher in obstetric care workers working in hospitals than in those working in health centers in Ethiopia [Yisma et al., 2013]. While the knowledge was higher among those working in hospitals, the utilization was more frequent in the health centers than in hospitals [Yisma et al., 2013]. The knowledge of partograph components was found to be poor among the auxillary nurses and midwives in Nigeria [Fawole et al., 2010]. More years of experience of a healthcare worker with the healthcare system in Nigeria was associated with better partograph utilization in terms of frequency and skills of partograph use [Opiah et al., 2012].

A majority of the studies $(\mathrm{n}=10)$ investigated knowledge of other components of basic EmOC and found: basic EmOC knowledge among the obstetric care workers was poor, EmOC knowledge and skills and confidence in providing the services was improved by training, higher knowledge scores of some life-saving techniques, such as neonatal resuscitation were associated with having performed the technique recently and with geographical location [Mirkuzie et al., 2014; Ameh et al., 2012; Haile-Mariam et al., 2012; Vivio et al., 2010; Kimberly et al., 2010; McAuliffe et al., 2013; Maramagi et al., 2004; Tita et al., 2006; Ijadunola et al., 2010; Ersdal et al., 2008]. However, type of healthcare worker such as being a nurse or a midwife did not have any associations with higher knowledge scores among healthcare workers in Ethiopia [Haile-Mariam et al., 2012].

Knowledge of active management of the third stage of labour (AMTSL) was fairly adequate among the healthcare workers in Zambia, although a few of the workers still practiced cord clamping and cutting, a procedure no longer recommended as part of AMTSL [Fawole et al., 2008]. Only a third of healthcare workers were aware of the fundal massage as a component of AMTSL [Vivio et al., 2010]. The knowledge and use of maternal ultrasound among midwives in Zambia was improved after training on maternal ultrasound [Kimberly et al., 2010]. Knowledge of basic EmOC among the different professions in SRH services in Nigeria was better among doctors compared to other professions, but no difference was found between age groups [Ijadunola et al., 2010]. Knowledge of symphysiotomy, a procedure performed to widen the pelvis in order to ease childbirth when there is a mechanical problem, was moderate among all participants in the Zimbabwean study and all could describe how to perform it [Ersdal et al., 2008]. In particular, rural midwives in Zimbabwe regarded the technique as a 
lifesaving one appropriate for remote areas, and more than half of them thought that it was necessary to be taught [Ersdal et al., 2008].

With regards to PMTCT, knowledge among nurses and midwives was moderate Nigeria, with knowledge and behaviour being positively correlated [Ndikom et al., 2007; Chi et al., 2004]. Knowledge of PMTCT among nurses and midwives who manages pregnant women living with HIV and AIDS in Nigeria was significantly better from those who did not manage pregnant women living with HIV and AIDS [Ndikom et al., 2007]. Knowledge of emergency contraceptive, on the one hand, was fair among the different cadre of healthcare workers in Uganda [Byamugisha et al., 2007]. On the other hand, 1 in 4 healthcare workers did not know the time limit within which EC was effective. Knowledge of postpartum infections and management of hypertensive complications was fairly poor among the healthcare workers in Mali [Traore et al., 2014]. However, high knowledge scores on immunization issues were found among healthcare workers in Nigeria [Ehiri et al., 2005].

With regards to continuous education, a model often used in the healthcare setting to continuously improve knowledge and skills of healthcare workers, nearly half of the participants in a study in Swaziland had no continuous education training and felt that they needed guidance or supervision on adolescent sexual and reproductive health care, although some of them wanted to be trained on post-abortion care while some wanted to be trained on how to perform abortions [Worku et al., 2013]. A small proportion of the healthcare workers wanted the government to train healthcare workers in adolescent sexual and reproductive health services [Worku et al., 2013]. In Nigeria however, a large proportion of healthcare workers (68\%) had adequate training in immunization, according to Ehiri et al., [2005].

\section{Attitudes toward women and adolescents seeking SRH services}

Negative attitudes towards provision of SRH services to adolescents in Ethiopia was significantly associated with healthcare workers' being married, lower levels of education, being a health extension worker, lack of training on RH services, and non-use of family planning by healthcare workers themselves [Tilahun et al., 2012]. A majority of healthcare workers in both Kenya (82\%) and Zambia (86\%) reported that they would advise adolescents to abstain from sex when they seek contraceptives [Warenius et al., 
2006]. With regards to provision of contraceptives to young women, healthcare workers had a minimum age restriction, as well as restrictions according to clients' marital status in Senegal [Sidze et al., 2014]. However, healthcare workers with more education and those who had received continuing education on adolescent sexuality and reproduction showed a tendency towards more youth- friendly attitudes and were in support of contraceptive use among adolescents [Warenius et al., 2006]. Furthermore, these two countries concurred with the notion of adolescent boys being taught the dangers of masturbation [Warenius et al., 2006]. On the positive attitudes of healthcare workers, a majority of the obstetric care workers in Zimbabwe agreed that adequate knowledge about prenatal services, accessible and acceptable services, affordable services, needsfocused prenatal services, prompt services and perceived benefits would influence women and adolescents' decisions to utilize prenatal services [Chaibva et al., 2010].

\section{Healthcare workers' cultural and religious beliefs regarding SRH services}

Religion was found to be a factor among healthcare workers in Swaziland as some healthcare workers reported that contraceptive use was against their religion and that youth should not indulge in sex [Mngadi et al., 2008]. Furthermore, masturbation is regarded as a sinful act and those who perform it are believed to experience mental disorders or fertility problems by a majority of healthcare workers in both Kenya $(\mathrm{n}=264)$ and Zambia $(\mathrm{n}=331)$ [Warenius et al., 2006]. In general, masturbation is considered a taboo in the southern region of SSA. Nurses and midwives disagreed that abortion should be provided to adolescent girls with unwanted pregnancies, on both cultural and religious grounds in Kenya (80\%) and Zambia (94\%) [Warenius et al., 2006]. Nearly a third (26\%) of the nurses and midwives in both countries reported that they would feel annoyed if an adolescent girl would report with abortion-induced related symptoms [Warenius et al., 2006].

\section{Availability of SRH services and resources}

In Ethiopia, availability of essential drugs, equipment and other supplies was unsatisfactory, and discrepancies were found between providers and clients with regards to services provided and services received [Worku et al., 2013. A majority of the facilities in Ethiopia did not function fully for EmOC as per their level, as well as important ANC components were incomplete and unsatisfactory [Worku et al., 2013]. Ndikom et al., (2007) found a significant relationship between behaviours and 
availability of resources. While facilities were adequately equipped with immunization supplies in Nigeria, essential drugs supply was inadequate in all centers; as well as emergency care facilities [Ndikom et al., 2007]. Various healthcare system constraints, such as human resources, availability of essential medical supplies including drugs, and challenges with the referral system were reported as key factors influencing decisionmaking for clinical care in Ghana [Oduro-Mensah et al., 2013].

Printed protocols and guidelines were the most commonly selected aids used in decision-making for clinical care and the availability of guidelines was associated with higher competency of physicians, health technicians, and obstetric nurses [OduroMensah et al., 2013]. However, study participants also reported healthcare systems' facilitators in decision-making for clinical care in Ghana. The facilitators included availability and consultation of workshop materials, expert advice and telephone calls to other colleagues, and access to various locally (institutionally) modified or developed guidelines and protocols e.g. guidelines on the management of postpartum [OduroMensah et al., 2013].

Contrary to Oduro-Mensah, et al., [2013], Implementation of clinical guidelines was less than optimal, with certain sections of the guidelines better implemented than others by healthcare workers in Rwanda [Leon et al., 2006]. Furthermore, higher competency in EmOC skills was linked to the availability of guidelines, which further enhanced competency of other healthcare workers with fewer than 10 years of experience in Mali [Traore et al., 2014].

\section{Other factors associated with provision of quality SRH services}

The major factors found to influence behaviour towards PMTCT services were mainly fear of getting infected, irregular supply of resources like gloves, goggles, and sharps boxes, and an irregular supply of water [Ndikom et al., 2007]. A majority of healthcare workers in Kenya were in favour of equal treatment for post abortion care (PAC) regardless of the age or marital status of clients [Evens et al., 2014]. All healthcare workers in Kenya deemed PAC services important. Sexual intercourse after marriage was supported by less than half of the healthcare workers in the Kenyan study [Evens et al., 2014]. Private doctors in South Africa considered caesarean section (CS) safer than a "normal" delivery, a convenience and financially enticing to the doctor [Chalmers et al., 
1992]. Public doctors believed that CS is performed by obstetricians due to a lack of proper (training) management of complications or difficult delivery in South Africa [Chalmers et al., 1992].

\section{Discussion}

This systematic review includes studies on the behaviors and personal determinants of healthcare workers pertaining to sexual and reproductive health from sub-Saharan Africa. Negative behaviours and attitudes of healthcare workers, as well as personal determinants, such as poor knowledge and skills of SRH services, and related factors, like unavailability of essential drugs and equipment are associated with provision of inadequate SRH services. Negative behaviours, including negative attitudes of healthcare workers regarding some SRH services, particularly for adolescents' SRH services were commonly reported in the studies included in this review [Tilahun et al., 2012; Warenius et al., 2006; Evens et al., 2014; Chalmers et al., 1992]. Healthcare workers' negative behaviours and attitudes are unlikely to encourage women in general to access and utilize SRH services, but more specifically young women.

With regards to adolescents specifically, it appears that nurses and midwives disapprove of adolescents' sexual activities and show reluctance in providing SRH services to adolescents who engage in sexual activities [Warenius et al., 2006]. Healthcare workers' negative behaviours and attitudes towards adolescents seeking SRH services, such as contraceptives are well documented in SSA, and have a negative effect on adolescents' access to and utilization of the services [Biddlecom et al., 2007; Chaibva et al., 2010; Zielinski et al., 2001; Tilahun et al., 2012; Warenius et al., 2006; Mngadi et al., 2008; Evens et al., 2014; Chalmers et al., 1992; Mannava et al., 2015]. Consequently, many adolescents continue to fall pregnant and for some, the pregnancy is unwanted [Bearinger et al., 2007; Mngadi et al., 2008; Raine et al., 2005; Sidze et al., 2014]. Furthermore, negative behaviours and attitudes of healthcare workers considerably affect women and adolescents' decision to access and utilize antenatal care services during pregnancy, including decisions to deliver the baby at a healthcare facility and post-natal care thereafter [Chaibva et al., 2010; Mannava et al., 2015]. This finding is a cause for concern, as contraceptive use by adolescents is needed to help reduce teenage 
pregnancy, and antenatal care is important to monitor pregnancy and helps to detect and prevent potential complications during pregnancy.

Evidence shows that healthcare workers' negative behaviours discourage women and adolescents from accessing and utilizing SRH services [Biddlecom et al., 2007; Glasier et al., 2006; Chaibva et al., 2010; Ngomane et al., 2012; Geary et al., 2014; Zielinski et al., 2001; Kaufman et al., 2001; Singh et al., 2005; Tilahun et al., 2012; Wood et al., 2006]. For example, healthcare workers' negative attitudes towards provision of contraceptives to adolescents hinder adolescents' access to and utilization of SRH services [Tilahun et al., 2012; Wood et al., 2006]. It is therefore clear that interventions which aim to address and change the negative behaviours and attitudes of healthcare workers in SRH services are likely to improve healthcare utilization, especially by adolescents.

Personal determinants, which most consistently appeared to be associated with adequate quality provision of SRH services at large, were knowledge and skills of SRH service components, and continuous education on SRH service components. Knowledge and skills of basic, yet necessary and life-saving components of SRH services is unsatisfactory, according to the studies included in this review. Continuous education, a method of keeping abreast with current and updated knowledge also appears to be a method least utilized by healthcare workers according to the studies included. This is concerning, given the poor knowledge and skills of SRH services among healthcare workers reported by the studies included in this review.

Knowledge of specific SRH components, such as the knowledge of partograph and the components of active management of the third stage of labour (AMTSL) are below optimum levels, and are not being fully implemented by some healthcare workers as per the WHO recommended guidelines of EmOC and AMTSL. These SRH components include the poor utilization of partograph during labour, the controlled cord clamping and cutting, and the fundal massage, which are all very useful and can save lives if fully and correctly implemented. This finding echoes findings from a study by Ueno et al., [2015] which investigated and evaluated the implementation of EmOC components in public hospitals where only two hospitals fully implemented the nine components of EmOC. Signal functions requiring skill like manual removal of placenta, removal of retained products, or vacuum extraction, were also not performed at all in the healthcare centre, 
and only three out of eight facilities provided anticonvulsants even though eclampsia is one of the leading causes of complications during labour and of maternal deaths [Ueno et al., 2015].

These components are crucial components of adequate and quality SRH services, and are essential to mother and child healthcare outcomes. Therefore, optimum knowledge and skills of both EmOC and AMTSL are necessary to improve maternal and child health, and reduce maternal and child mortality and morbidity. Furthermore, evidence synthesized in this review show that healthcare workers have limited knowledge of emergency contraceptives (EC) and how it works. This is also a cause for concern as knowledge of EC and its provision is particularly important to prevent unwanted or unplanned pregnancies. This finding is similar to previous research, where knowledge of EC was relatively poor among healthcare workers [Byamugisha et al., 2007; Raine et al., 2005; Gold et al., 1997; Mane et al., 2015]. Adolescents are the most frequent group of people who tend to use and or need EC to prevent unwanted pregnancies after having had unsafe sex [Raine et al., 2005]. However, if healthcare workers do not know how EC work and when it is best to provide it, it is unlikely that they would encourage adolescents to seek this service or provide it should the need arise [Gold et al., 1997; Mane et al., 2015; Lawani et al., 2014]. This further puts limitations for adolescents to access and utilize services for their SRH needs.

Healthcare systems related factors, such as a lack of resources including essential drugs and equipment, also have a relation to inadequate quality provision of SRH services. Inadequate availability of essential drugs and other equipment not only impede access to and utilization of services, but also the provision of adequate quality service in SRH services. Factors such as a shortage of staff, drugs and equipment, and limited skills have been identified in the literature as possible reasons why quality of SRH services is not optimal, for example EmOC requiring skills are the least performed by healthcare workers in SRH services [Ersdal et al., 2008; Leon et al., 2007]. Thus, to improve quality service provision, and access to and utilization of services in SRH, sufficient availability of resources is crucial.

It is worth noting that SSA countries are different from each other, but also share a few similarities, such as poor infrastructure, limited healthcare resources, including limited 
human resources in healthcare settings. With regards to culture and religion, some countries in SSA are more sensitive than others. For example, Swaziland is more sensitive to religion than culture and thus, behaviours of many healthcare workers in this country is informed or based on their religious beliefs. While in SA and Ethiopia for example, culture and traditional practices are more value and inform the behaviours of most healthcare workers [Tilahun et al., 2012; Wood et al., 2006; Mngadi et al., 2008]. Both the differences and similarities provide useful information when designing and developing interventions to improve the negative behaviours of healthcare workers in SSA countries. Furthermore, these differences and similarities helps in identifying countries where maximum efforts should be placed, and which specific behaviours should be prioritized when designing the interventions for improvement.

\section{Limitations}

Many of the studies included came from northern regions compared to the southern regions of SSA, although it is well known that inadequate SRH services are predominantly vast in the southern region. Methodologically, the majority of the studies included in this review were descriptive cross-sectional studies with a strong heterogeneity. This makes it hard to pool results from different studies. Moreover, it is possible that some interesting findings are in the grey literature, which this review did not access. The other limitation is the lack of information on adolescent specific obstetric care. However, this is not surprising, as obstetric care in general has been tailored around the general population of women and not adolescents.

The results of this review are not necessarily generalizable to each and every country in the SSA region due to the limited number of studies supporting this review's findings. Hence, caution is warranted to draw general conclusions. However, the limited number of studies in SRH services in SSA emphasizes the need for more research in this field in order to draw conclusive and generalizable findings. Furthermore, findings of this review can be used to some extent to highlight specific areas needing intervention, but additional needs assessment maybe needed. It is clear that more research still needs to be done to uncover healthcare workers' behaviours and associated factors which have a negative effect on access to and utilization of SRH, and quality of care in this field. 


\section{Implication for practice}

Healthcare workers behaviours, personal values, and morale significantly hinder the provision of adequate quality SRH services to young people, especially to adolescent girls. Adolescents' SRH services need to be youth-friendly, and served by welcoming healthcare workers who have a positive attitude towards adolescents seeking SRH services. This kind of environment will further equip adolescents seeking SRH services with the knowledge and information they require to make informed choices regarding their SRH.

It has been shown that interactive forms of continuous education sessions on sexual and reproductive healthcare that enhance participant activity and provide the opportunity to practice skills can effect change in professional practice and, improve health care outcomes [Peck et al., 2000; Davis et al., 1999]. The findings in this review of doctors having better knowledge of specific SRH components compared to nurses and midwives, highlights the importance of further education and training among nurses and midwives in providing SRH services.

Basic EmOC signal functions, as described by the WHO, cannot be fully performed if a health facility is inadequately equipped with necessary resources to carry all the signal functions. The availability, continuous and frequent utilization of SRH resources, such as the partograph are very important and cannot be over emphasized as they play a crucial role in maternal and child health outcomes. Therefore, increasing essential supplies and equipment in health facilities is very important to strengthening the healthcare system and improving health outcomes.

\section{Implication for policy}

Continuous SRH education and training is necessary to adequately provide quality services, and therefore needs to be incorporated and enforced into the healthcare systems policies for professional development. The importance of comprehensive knowledge and skills to adequately care for women, newborn, children, and adolescents should be prioritized as it is critical in SRH services. Reducing maternal morbidity and mortality rates, including deaths of newborn, and children under five years of age, are a priority in the SDG 2016-2030. To ascertain that this specific target is achieved in SSA, 
serious efforts and investments towards adequate quality healthcare services are needed.

Religion, a socio-demographic factor associated with many behaviours, is commonly acknowledged in the SSA region and requires attention to assist healthcare workers who find themselves in conflict of their religion when rendering some SRH services. Healthcare workers in SRH services may benefit from policy guidelines and instructions pertaining to religious beliefs and clarifying the role of healthcare providers in this regard. Healthcare workers should be able to provide SRH services to women and adolescents when they require them, irrespective of their own personal views and religious beliefs.

\section{Implications for research}

There is a need for an in-depth understanding of the negative behaviours and attitudes of healthcare workers in SRH services, and their associated factors in order to develop interventions that will address them and consequently stimulate access to and utilization of SRH services by women and young people in SSA. Interventions to promote positive behaviours and attitudes of healthcare workers in SRH services are needed. Furthermore, future studies should be intervention-based focusing on changing the known and reported negative behaviours and attitudes of healthcare workers in SRH services into positive ones. Such intervention studies may include changing the negative behaviours and attitudes of healthcare workers with regards to young women's use of contraceptives in some parts of SSA, taking into account the contextual differences in SSA regions. An SRH specific evaluation tool to regularly monitor and evaluate knowledge and skills of healthcare workers in SRH services would be useful to ensure continuous education and training, and improved SRH knowledge and skills.

\section{Conclusions}

Knowledge of SRH services, including basic EmOC is insufficient among healthcare workers in SSA. Healthcare workers also seem to experience a conflict between their attitudes towards the sexual behaviors and their role as providers of support and information about SRH issues to adolescents in particular. The more knowledgeable the healthcare worker is the better is his/her behaviour. Positive behaviours and attitudes 
are highly likely to influence women and adolescents' utilization of SRH services for their needs. Healthcare workers in SRH services have to be clinically trained and skilled on comprehensive sex education and other related SRH issues for women and adolescents specifically. Access to and utilization of adequate, quality SRH services will most likely reduce teenage pregnancy and the illegal and unsafe abortions in the SSA region. A continuous education program for healthcare workers in SRH services can be feasible and potentially successful, given the interest expressed by healthcare workers. 


\title{
CHAPTER 4
}

\section{Published as}

Jonas K, Reddy P, van den Borne B, Sewpaul R, Nyembezi A, Naidoo P, Crutzen R. Predictors of nurses' and midwives' intentions to provide maternal and child healthcare services to adolescents in South Africa.

\begin{abstract}
Background: Adolescent mothers are at a much higher risk for maternal mortality compared to mothers aged 20 years and above. Newborns born to adolescent mothers are also more likely to have low birth weight, with the risk of long-term effects such as early onset of adult diabetes than newborns of older mothers. Few studies have investigated the determinants of adequate quality maternal and child healthcare services to pregnant adolescents. This study was conducted to gain an understanding of nurses' and midwives' intentions to provide maternal and child healthcare and family planning services to adolescents in South Africa.

Methods: A total of 190 nurses and midwives completed a cross-sectional survey. The survey included components on demographics, knowledge of maternal and child healthcare $(\mathrm{MCH})$ and family planning (FP) services, attitude towards family planning services, subjective norms regarding maternal and child healthcare and family planning services, self-efficacy with maternal and child healthcare and family planning services, and intentions to provide maternal and child healthcare and family planning services to adolescents. Pearson's correlation analysis was conducted to determine the association between knowledge, attitude, subjective norms, self-efficacy, and intention variables for FP and MCH services. A 2-step linear regression analysis was then conducted for both FP and $\mathrm{MCH}$ services to predict the intentions to provide $\mathrm{FP}$ and $\mathrm{MCH}$ services to adolescents.
\end{abstract}

Results: Self-efficacy to conduct MCH services $(\beta=.55, \mathrm{p}<0.01)$ and years of experience as a nurse- midwife $(\beta=.14, \mathrm{p}<0.05)$ were associated with stronger intentions to provide the services. Self-efficacy to provide FP services $(\beta=.30, p<0.01)$ was associated with stronger intentions to provide FP services.

Conclusions: Self-efficacy has a strong and positive association with the intentions to provide both MCH and FP services, while there is a moderate association with attitude and norms. There is a need to improve and strengthen nurses' and midwives' self- 
efficacy in conducting both MCH and FP services in order to improve the quality and utilization of the services by adolescents in South Africa. 


\section{Introduction}

The high maternal and neonatal mortality rates in developing countries are a major public health problem, especially in developing countries [Ueno et al., 2015]. SubSaharan Africa is among the regions affected by high number of maternal mortality at 201000 in 2015 [WHO, 2014] and was reported to have contributed to $62 \%$ of all maternal deaths worldwide in 2013 [WHO, 2014; Accorsi et al., 2010]. Most of these deaths are a result of preventable causes [Accorsi et al., 2010]. Maternal mortality ratio in South Africa (SA) are high at 138 per 100000 live births [World Bank Data, 2015].

Adolescent mothers are at a much higher risk for maternal mortality [WHO, 2014]. Nearly 16 million girls aged 15 to 19 years give birth every year and about 2.5 million of these births occur to girls aged under 16 years in low- and middle- income countries (LMICs) [World Bank Data, 2015; Neal et al., 2015; Martin et al., 2012]. Pregnancy and childbirth complications are the second cause of death among 15 to 19 year olds globally [WHO, 2014]. Early childbearing increases the risks for maternal and neonatal mortality [Neal et al., 2015].

In LMICs, infants born to mothers under 20 years of age face a 50\% higher risk of being still born or dying in the first few weeks versus those born to mothers aged 20-29 [WHO, 2014; Neal et al., 2015]. Newborns born to adolescent mothers are also more likely to have low birth weight, with the risk of long-term effects such as early onset of adult diabetes. In addition, birth outcomes for very young adolescent mothers are particularly poor in terms of increased rates of low birth weights [Jewkes et al., 2009; Chigona et al., 2007]. However, very few studies have investigated the determinants of birth outcomes of pregnant adolescents and adolescent mothers in maternal and child healthcare (MCH) services in SA.

Whilst inadequate provision of MCH services to pregnant adolescents and adolescent mothers does not necessarily cause poor birth outcomes, adequate quality provision of these can improve outcomes of both the mother and her newly born infant. Negative attitude of nurses and midwives, lack of respect for the birthing mothers' views, and, with adolescents particularly, stigmatized sexual activity by the nurses are some of the barriers reported to influence non-utilization of FP and MCH services, such as antenatal 
care, and contraceptive use in [Ngomane et al., 2012]. Lack of youth friendly, dedicated space and youth-friendly trained staff were also reported as barriers to accessing FP services by youth [Geary et al., 2014]. Fears of being scolded and shouted at, as well as assumptions of being promiscuous by the nurses are specific and serious barriers pertaining to adolescents' utilization of FP and MCH services [Geary et al., 2014; Wood et al., 2006]. Other reported barriers include the perceived compassion and carrying of traditional birth attendants in comparison to nurses and midwives [Ngomane et al., 2012].

Behaviour of healthcare professionals attending to pregnant women and their newborns is of critical importance for the adequate quality of MCH services: it is one of the factors contributing to non-utilization of MCH services by adolescents [Chaibva et al., 2010]. Nurses and midwives' negative behaviour such as shouting at and scolding clients, is also reported to discourage young people from attending clinics or for followup visits [Biddlecom et al., 2007; Glasier et al., 2006], although little is known of the underlying determinants. According to the Theory of Planned Behaviour (TPB), an individual's behaviour as well as the extent to which he or she can control the behaviour is determined by his or her beliefs about having the necessary skills and resources to perform the behaviour [Fishbein et al., 2010]. Thus, adopting any behaviour, both desirable and undesirable behaviours, requires the capacity and confidence in one's ability to attempt the behaviour, that is, self-efficacy or perceived behavioural control. Constructs of TPB have been well documented and researched in their applicability in predicting many behaviours. A meta-analytic review showed that high perceived behavioural control (PBC) and self-efficacy were the strongest predictors of most behaviours, including intentions to perform the behaviours [Armitage et al., 2001]. TBP constructs have also been used to predict behaviours associated with unhealthy sexual and reproductive behaviours, such as poor contraceptive compliance. Condom use among adolescents for example, was associated with high self-efficacy in using the condom [Reddy et al., 2003].

Hence, constructs of TPB might also be associated with healthcare workers' behaviours related to providing family planning (FP) and $\mathrm{MCH}$ services to adolescents. The selfefficacy construct for instance, is an important construct for healthcare providers who believe that they cannot provide certain FP and MCH services adequately. Knowledge 
and confidence in using partograph, a basic component of managing labour in $\mathrm{MCH}$ services, was associated high utilization of partograph among healthcare providers [Wakgari et al., 2015; Okokon et al., 2014; Yisma et al., 2013].

Therefore, the focus of this study will be on identifying the predictors of the intention to provide family planning (FP) and MCH services, as intention is an important predictor of behaviour. In this study, FP services were defined as nurses' and midwives' activities and communications to regulate and or prevent conception among adolescents through the practice of contraception or other methods of birth control [WHO, 2014]. MCH services were defined as nurses and or midwives activities and communications given to expectant adolescents and adolescent mothers that is aimed at enhancing their health and that of their unborn baby and the infant [WHO, 2014]. Nurses are all healthcare professional with a nursing qualification, while midwives are nurses who are certified as qualified midwives.

Determining the psychosocial predictors of nurses' and midwives' intention to provide MCH services to adolescents will assist in (1) identifying the problems hindering the provision of adequate quality $\mathrm{MCH}$, including FP services to adolescents, (2) providing information to develop and test targeted behaviour change interventions for nurses and midwives in SA, and, ultimately, (3) improving the current maternal and infant health outcomes. Thus, the aim of this study was to determine nurses' and midwives' predictors of intention to provide FP and MCH service to adolescents in SA.

\section{Methods}

\section{Research design}

A quantitative cross-sectional study was conducted to gain an understanding of the nurses and midwives intentions to provide FP and $\mathrm{MCH}$ services to adolescents. A researcher-developed structured questionnaire was used to obtain information from nurses and midwives in maternal and child healthcare services in the nine provinces in SA. The questionnaire was partly based on constructs derived from Ajzens' Theory of Planned Behaviour (TPB) [Fishbein et al., 2010; Ajzen, 1991]. Thus, the questionnaire included questions about attitude, subjective norms, self-efficacy, and intentions to provide FP and MCH services. 


\section{Sampling}

The sampling frame consisted of all public health facilities that provide maternity and infant healthcare services in both urban and rural settings of SA. These facilities were stratified into two (2) categories: 1. Public Health Clinics, and 2. Community Day Centres (CDC), Community Health Centers (CHCs) and district hospitals. In this study, CHC is collectively used to group together the CHCs, CDCs and district hospitals as they all conduct secondary level of care compared to clinics which only conducts primary level of care. A representative sample from each stratum was selected using the probability proportional to size approach [Statistics SA, 2012a]. The sampling frame consisted of 147 clinics and 34 CDCs, CHCs and district hospitals.

All nine provinces of SA were included in the selection process in order to reflect the diverse demographic and socio-economic profile of the country. Nurses and midwives were recruited at the facility level with the assistance of facility managers. Depending on the size of the facility either all the nurses and midwives or a sample of them were studied. The inclusion criteria for nurses and midwives was: all the nurses whose work included family planning services, caring for pregnant women, delivering babies; and all midwives were eligible to participate in the study. Not all nurses and midwives in these units were available to complete the questionnaire due to the shortage of healthcare professionals in the country, leaving them having to deal with high patient load at the clinics. All nurses and midwives working in MCH and FP units were eligible and recruited to complete the questionnaire. Out of the 250 nurses and midwives that were approached, only 190 agreed to participate, giving a 76\% response rate. Work demands and patient overload or the busyness of the clinic were the most common reason for not participating in the study. In other words, the study used a convenience sample of nurses and midwives. The sample size depended on those who were available to participate in the study at the time of data collection in their facility.

\section{Data collection instruments}

Nurses and midwives completed a self-administered questionnaire measuring the determinants of MCH services, and family planning health services behaviour, including demographics as described below. The questionnaire was piloted in a non-study sample and necessary adaptations were made before the study was implemented. The final version of the questionnaire can be found in Supplementary file 1_Questionnaire. 


\section{Demographic questionnaire}

Background information: name of the province, district, location, facility and the type of facility were asked. The gender, participants' date of birth, position at the facility, the number of years in the position, and education was asked. The variables were coded as following: location ( $1=$ Urban, $2=$ Rural), gender ( $1=$ Male, $2=$ Female), position at the facility (1=Registered Nurse, 2=Registered Midwife, 3=Staff Nurse, 4=Enrolled Nurse and Other), type of facility ( $1=$ Clinic, $2=\mathrm{CHC}$ ). Education was ( $1=$ Degree in Nursing) and (2= Diploma in Nursing and Other). For the purposes of this study, position at facility was grouped into (1= Registered Midwives, $2=$ Registered nurses/Enrolled nurse/Staff nurse, and $3=$ other: nurses who were neither registered midwives nor registered nurses).

\section{Knowledge of family planning (FP) services}

Knowledge of FP services is a thirteen-item subscale which assesses nurses and midwives' knowledge of FP services in SA. The statements for the measurement of knowledge of FP services were extracted from the National Contraception Clinical Guidelines: A companion to the National Contraception and Fertility Planning Policy and Service Delivery Guidelines [Motsoaledi et al., 2012]. The statements were provided in the questionnaire where the nurse-midwife was required to confirm whether the statement was true, false or they don't know. Sample statements are: 1. Combined oral contraceptive pills should be taken at the same time each day, 2. In SA, any female can get an abortion by simply requesting with no reasons given if she is less than 13 weeks pregnant. Scoring options were coded as ( $1=$ True), ( $2=$ False), and ( $3=$ I don't know) and the total score was calculated based on the number of items answered correctly.

\section{Knowledge of maternal and child healthcare (MCH) services}

Knowledge of $\mathrm{MCH}$ services is an eighteen-item subscale which assesses nurses and midwives' knowledge of $\mathrm{MCH}$ services in SA. Knowledge of MCH services questions were extracted from the Guidelines for Maternity Care in South Africa (a confidential draft document then), which is now currently the official manual for clinics, CHC and district hospitals in SA [DoH, 2015]. Similar to the knowledge of family planning services 18 statements were provided to the nurses and midwives and were asked to confirm whether the statement was true, false, or they don't know. Sample items were: 1 . Family planning services are a basic component of $\mathrm{MCH}$ services, 2. Tetanus immunization is 
not compulsory for a pregnant woman in SA. Scoring options were coded as (1= True), $(2=$ False), and ( $3=$ I don't know) and the total score was calculated based on the number of items answered correctly.

\section{Attitudes towards providing FP services}

Attitude toward providing FP services is a ten-item subscale which assesses nurses and midwives' attitudes towards providing FP services to adolescents in SA. Nurse and Midwives were asked how important it is to them to discuss certain family planning topics with the adolescents in their facility. Sample items were: 1 . How important is it to you to discuss injectable contraceptives with adolescents? 2. How important is it to you to discuss termination of pregnancy (TOP) and choices on TOP with adolescents? A Likert scale of 1-5 was used to assess the importance of discussing the topics, where $1=$ Very unimportant, 2= Unimportant, 3= Neutral, 4= Important, 5= Very important).

\section{Subjective norms towards providing FP and MCH services}

Subjective norms subscale is a six-item (for FP) and 5-item (for MCH) subscale which assesses general norms of the nurses and midwives' colleagues at the facility they work in. Nurses and Midwives were asked how strongly they agreed with certain FP and MCH statements regarding their colleagues' views. The items were 6 for FP and 5 for MCH services. A Likert scale was also used to assess their subjective norms for FP and MCH separately. Sample items were: 1 . Most of your colleagues think that you should provide female condoms to adolescent girls (for FP services), 2. Most of your colleagues think that you should discuss delivery options with pregnant adolescent girls (for MCH services). Scoring options were 1= Strongly disagree, 2= Disagree, $3=$ Neutral, 4= Agree, $5=$ Strongly agree).

\section{Self-efficacy to provide FP and MCH services}

Self-efficacy is one's belief in his or her capabilities to perform a recommended behaviour [Bandura, 1994]. In this study, self-efficacy is defined as the nurses' and midwives' beliefs and confidence in themselves performing a recommended FP and MCH services for adolescents in SA. Nurses and Midwives were asked to indicate how confident they were to provide FP services and MCH services. The total items were 7 for FP and 10 for MCH services. To measure FP self-efficacy a sample question was: how confident are you that you will be able to provide injectable contraceptives to adolescent 
girls? To measure MCH self-efficacy a sample question was: how confident are that you will be able to provide immunization for diphtheria, tetanus, and pertussis to pregnant adolescents. Scoring options were $1=$ Not confident at all, $2=$ Not confident, $3=$ Unsure, $4=$ Confident, $5=$ Very confident.

\section{Intention to provide FP and MCH services}

Intention is a sixteen-item (for FP services) and ten-item (for MCH services) which assesses nurses and midwives' intentions to provide family planning services to adolescents in SA. Nurses and Midwives were asked to indicate whether they intended to provide certain FP and MCH services. Sample items were: the next time an adolescent girl needs TOP, I intend to provide (for FP services), and the next time a pregnant adolescent needs advice on delivery options, I intend to provide the advice (for MCH services). The total number of items was 16 for FP services and 10 for MCH services. The Likert scale scoring options were $1=$ Definitely not, $2=$ Not, $3=$ Unsure, $4=$ Yes, $5=$ Definitely yes.

\section{Data analysis}

Data were analyzed using SPSS version 21 [IBM SPSS, 2012]. Descriptive statistics were conducted to get an overview of the sample characteristics. Mean scores were also calculated in order to gain more information about the nurse- midwives' personal determinants regarding adolescents' $\mathrm{MCH}$ and FP services. Pearson's correlation analysis was then conducted to determine the association between knowledge, attitude, subjective norms, self-efficacy, and intention variables for FP and MCH services. The strengths for Pearson's correlation were classified as weak $(.10 \leq \mathrm{r}<.30)$, moderate $(.30$ $\leq \mathrm{r}<.50)$ or strong $(\mathrm{r} \geq .50)$ [Rosenthal, 1996]. All variables, including the demographics were then taken into multiple linear regression analysis to predict the intentions to provide FP and MCH services to adolescents by the nurses and midwives in this study. Intentions to provide FP and MCH services are the outcome variables for the linear regression analysis.

Prior to the linear regression analysis, exploratory factor analysis was conducted to assess the items making up the scales in order to group together items that were closely related [Pallant, 2013]. Thirteen items from the knowledge of FP services, and 18 items from the knowledge of MCH services were put through factor analysis in SPSS. Kaiser- 
Mayer-Oklin (KMO) test for the FP knowledge subscale met the recommended value at .6 with the Bartlett's test reaching the significance level, $\mathrm{p}<0.01$, and the KMO value for MCH knowledge subscale was more than the recommended 6 with a significant Bartlett's test, $\mathrm{p}<0.01$ [Pallant, 2013]. Ten items of FP attitudes, 6 items on subjective norms for FP and 5 items for MCH, 7 items self-efficacy for FP and MCH services, and 16 items on intentions to provide FP and MCH services were also put through the factor analysis. All these subscales were above the minimum recommended .6 value of the KMO with a significant Bartlett's test $\mathrm{p}<0.01$ [Pallant, 2013]. Kaiser-Mayer-Oklin and Bartlett's tests were used to assess the suitability of the data for the planned analysis to ascertain that no assumptions were violated, as well as the appropriateness of the factor analysis that was used to assess the items making up the scales used [Pallant, 2013]. The KMO values confirmed that the variables used in this study were fit for further analysis, which the study intended to conduct.

A 2-step linear regression analysis was conducted for both FP and MCH services. In step 1, only demographics: Location (urban or rural), position of the staff, education level, and years of experience were added into the FP and MCH regression models. In step 2, demographics and the psychosocial variables: FP knowledge, FP attitude, FP norms, and FP self-efficacy were added into the FP model. MCH psychosocial variables: $\mathrm{MCH}$ knowledge, MCH norms, and MCH self-efficacy were added into the MCH model.

Collinearity statistics showed that the variables entered into the linear regression model did not violate the multi-collinearity assumptions. All variables for both FP and MCH services obtained an acceptable variance inflation factor (VIF) that is below 10, the cut off value [Pallant, 2013; Field, 2009]. Only variables with a p- value of less than 0.05 ( $\mathrm{p}<$ 0.05 ) were considered significant variables in this study.

\section{Results}

\section{Sample characteristics}

The total sample consisted of 190 nurses and midwives from the nine (9) provinces of SA. Only ten (5.2\%) of the nurses and midwives were male, the majority was female 183 (94.8\%). The mean age of the nurses and midwives was 46.0 ( $\mathrm{SD}=10.0$ ), with 34 (21.4\%) being 30 - 39 years of age; 46 (28.9\%) being 40 - 49 years of age; and 52 $(32.7 \%)$ being 50 - 59 years old. Majority of the nurses and midwives, 129 (73.3\%) 
worked in a rural setting. The Public Health Clinic was the common type of facility many worked in (81.2\%), while only $18.8 \%$ worked in CHCs. Nearly a quarter of them $(25.3 \%)$ had a degree in nursing and the majority (74.7\%) had a diploma in nursing or other levels of education. Further sample characteristics are described below in Table 1.

Table 1. Demographic characteristics of the health care workers $(n=190)$

\begin{tabular}{|c|c|c|c|}
\hline & & Count (n) & Percent (\%) \\
\hline \multirow{3}{*}{$\begin{array}{l}\text { Total } \\
\text { Gender }\end{array}$} & & 190 & 100 \\
\hline & Male & 9 & 4.8 \\
\hline & Female & 179 & 95.2 \\
\hline \multirow[t]{5}{*}{ Age group } & $20-29$ & 12 & 7.8 \\
\hline & $30-39$ & 32 & 20.8 \\
\hline & $40-49$ & 45 & 29.2 \\
\hline & $50-59$ & 51 & 33.1 \\
\hline & $>=60$ & 14 & 9.1 \\
\hline \multirow{9}{*}{ Province } & Gauteng & 12 & 6.6 \\
\hline & Mpumalanga & 16 & 8.8 \\
\hline & Western Cape & 21 & 11.6 \\
\hline & Eastern Cape & 23 & 12.7 \\
\hline & Northern Cape & 27 & 14.9 \\
\hline & Free State & 24 & 13.3 \\
\hline & Kwazulu-Natal & 17 & 9.4 \\
\hline & Limpopo & 26 & 14.4 \\
\hline & North-West & 15 & 8.3 \\
\hline \multirow{2}{*}{$\begin{array}{l}\text { Type of facility where the } \\
\text { nurses and midwives work }\end{array}$} & Clinic & 147 & 81.2 \\
\hline & $\mathrm{CHC}$ & 34 & 18.8 \\
\hline \multirow{2}{*}{$\begin{array}{l}\text { Facility Setting where the } \\
\text { nurses and midwives work }\end{array}$} & Urban & 53 & 28.3 \\
\hline & Rural & 134 & 71.7 \\
\hline \multirow{3}{*}{$\begin{array}{l}\text { Current position of the nurses } \\
\text { and midwives at the facility } \\
\text { where they work }\end{array}$} & Registered Midwife & 57 & 30.0 \\
\hline & $\begin{array}{l}\text { Registered Nurse/Staff } \\
\text { Nurse/ Enrolled Nurse }\end{array}$ & 104 & 54.7 \\
\hline & $\begin{array}{l}\text { Other: nurses who are } \\
\text { neither registered midwives } \\
\text { or nurses }\end{array}$ & 29 & 15.3 \\
\hline \multirow[t]{6}{*}{ Years of experience } & $1-5$ years & 49 & 28.0 \\
\hline & 6-10 years & 46 & 26.3 \\
\hline & $11-15$ years & 27 & 15.4 \\
\hline & $16-20$ years & 15 & 8.6 \\
\hline & 21-30 years & 34 & 19.4 \\
\hline & $>=31$ years & 4 & 2.3 \\
\hline
\end{tabular}




\begin{tabular}{llcc}
\hline Educational background & $\begin{array}{l}\text { Degree in Nursing } \\
\text { Diploma in Nursing and } \\
\text { Other: nurses with less than } \\
\text { a degree or diploma } \\
\text { qualification }\end{array}$ & 130 & 27.0 \\
\hline
\end{tabular}

\section{Mean knowledge scores for FP and MCH services}

The mean knowledge score of FP services for all nurses and midwives was 9.6 (SD = 1.7), where 25.6\% scored 5-8 (lowest scores), 61.9\% scored 9-11 (median scores) and $12.5 \%$ scored 12-13 (highest scores). With regards to $\mathrm{MCH}$ services, the mean knowledge score among nurses and midwives in the study was 16.2 (SD =1.9). Nurses and midwives had moderately high knowledge of MCH practices with 55.3\% achieving a mean score of 17 - 18 (the highest scores), and 38.8\% achieving a mean score of $13-16$ (median scores).

Correlations between nurses and midwives' psychosocial determinants and intentions to provide FP and MCH services

The relationship between nurses and midwives' psychosocial determinants and intentions to provide FP and MCH services were investigated. Table 2 shows the results of Pearson's correlation analysis. No significant correlations were found between FP knowledge, MCH knowledge and the intentions to provide FP and MCH services to adolescents. Positive correlation was observed for FP attitudes ( $r=.29, \mathrm{p}<0.01)$, and FP norms $(\mathrm{r}=.27, \mathrm{p}<0.01)$. The moderate correlations between FP attitude and FP norms, show that a more positive FP attitude, better FP norms, are more likely to increase the nurse- midwives' intentions to provide FP services to adolescents in SA. There was a strong correlation between FP self-efficacy and intentions to provide FP services ( $\mathrm{r}=$ $.44, \mathrm{p}<0.01$ ). This positive correlation suggest that the more confident the nursemidwives are in providing FP services to adolescents the higher the intentions to provide the FP services to adolescents. A moderate positive correlation between $\mathrm{MCH}$ norms and intentions to provide MCH was also observed $(r=.27, \mathrm{p}<0.01)$. MCH selfefficacy correlation with intentions to provide $\mathrm{MCH}$ was also a positive correlation $(\mathrm{r}=$ $.58, \mathrm{p}<0.01$ ). MCH self-efficacy had a fairly strong correlation indicating that the more confident the nurse or midwife is (self-efficacy) with conducting MCH services to adolescents the more likely that he or she intends to provide MCH services to adolescents. 
Table 2. Pearson correlations between psychosocial determinants and intentions to provide family planning (FP) services and mother and child healthcare (MCH) services

\begin{tabular}{lcc}
\hline & FP Intention & MCH Intention \\
Psychosocial predictors & r (95\% CI $)$ & r (95\% CI) \\
\hline
\end{tabular}

FP knowledge

-.12 (95\% CI: $-0.28-0.05)$

FP attitudes $.29 * *(95 \%$ CI: $0.10-0.46)$

FP norms $.27^{* *}(95 \%$ CI: 0.12- 0.42)

FP self-efficacy $.44^{* *}(95 \%$ CI: $0.29-0.56)$

MCH services knowledge

MCH norms

MCH self-efficacy
-.05 (95\% CI: $-0.21-0.11)$

$.27 * *(95 \%$ CI: $0.13-0.41)$

$.58 * *(95 \%$ CI: 0.47 - 0.67)

${ }^{* *}$ Correlation is significant at $\mathrm{p}<0.01$

Multivariate predictors of nurse- midwives intentions to provide FP and MCH services to adolescents

A hierarchical linear regression analysis was conducted in Table 3 and Table 4 below. Table 3 Model: in step 1, demographic characteristics we fed into the regression model to determine the variables predicting intentions to provide FP services among nurses and midwives. None of variables were significant in this step. In step 2 all variables were fed into the regression model including those in step 1 . None of the variables that were included in step 1 were significant in step 2. One variable in step 2, FP Self-efficacy variable was significant, with $p<0.01$ (see Table 3 ). FP self-efficacy $(\beta=.30, p<0.01$ ) was the only significant positive predictor of intentions to provide FP services. This suggests that a stronger confidence in conducting FP services to adolescents is correlated with stronger intentions to provide FP services. 
Table 3. Regression analysis predicting intentions to conduct FP services among nurses and midwives

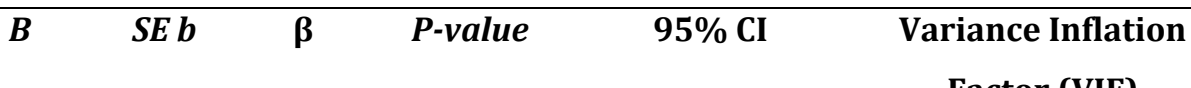

Factor (VIF)

\section{Step 1}

Constant

41.88

Location

$-.90$

.91

$-.11$

.32

$-2.71-0.89$

1.08

Staff position

$.16 \quad .28$

.06

.57

$-0.39-0.73$

1.07

Staff education

.86

.93

.10

.36

$-0.98-2.72$

1.06

Years of

experience

$-.0$

$.26 \quad-.03$

.79

$-0.58-0.45$

1.04

\section{Step 2}

Constant

Location

$$
-1.36
$$

.82

$-.16$

.10

$-2.98-0.27$

1.13

Staff position

$.18 \quad .25$

.07

.47

$-0.32-0.68$

1.08

Staff education

$.97 \quad .83$

.11

.24

$-0.68-2.63$

1.08

Years of

experience

$\begin{array}{lll}.16 & .24 \quad .06\end{array}$

.50

$-0.31-0.63$

1.11

FP Knowledge

$-.33 \quad .21$

$-.15$

.12

$-0.74-0.08$

1.04

FP Attitude

$.23 \quad .12 \quad .19$

.06

$-0.01-0.47$

1.21

FP Norms

$.12 \quad .15$

.16

$-0.07-0.42$

FP Self-efficacy

$\begin{array}{lllll}.49 & .18 & .30^{* *} & <.001 & 0.14-0.85\end{array}$

Step 1: $\Delta R^{2}=0.021 ; \Delta F=0.47 ; p>0.05$

Step 2: $\Delta R^{2}=0.25 ; \Delta F=7.32 ; \mathrm{p}<0.01$

${ }^{* *} \mathrm{p}<0.01$

With regards to MCH services, Table 4 Model: the same process was followed for model predicting intentions to provide MCH services among the nurses and midwives. Again, none of the variables in step 1 were significant. However, years of experience $(\beta=.14, p$ $<0.05)$ and a stronger confidence in conducting MCH services- MCH self-efficacy ( $\beta=$ $.55, \mathrm{p}<0.01$ ) were correlated with providing MCH services to adolescents (see Table 4).

Considering the Benferroni adjustment where $\mathrm{p} \leq 0.0071$ (i.e., 0.05/7) is significant, both FP self-efficacy and MCH self-efficacy remain significant predictors of intentions to provide FP services and MCH services, respectively. 
Table 4. Regression analysis predicting intention to conduct MCH services among nurses and midwives

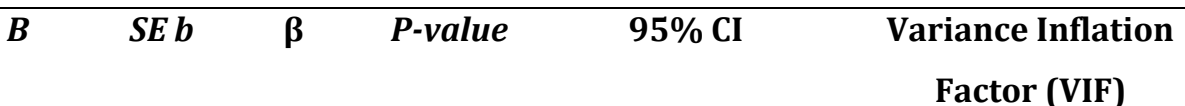

Factor (VIF)

\section{Step 1}

Constant

46.82

Location

.06

Staff position

.17

.01

.91

$-1.03-1.15$

1.08

Staff education

.17

.57

.05

.52

$-0.23-0.45$

1.07

Years of

experience

$\begin{array}{lll}.31 & .16\end{array}$

.76

$-0.94-1.29$

1.06

Step 2

Constant

19.45

Location

$-.24$

.45

$$
-.04
$$

.60

$-1.14-0.66$

1.12

Staff position

$.06 \quad .14$

.03

.66

$-0.22-0.34$

1.09

Staff education

.33

.46

.05

.48

$-0.58-1.24$

1.07

Years of

experience

.27

$.13 \quad .14^{*}$

.04

$0.01-0.52$

1.07

MCH Knowledge

$\begin{array}{lll}.01 & .10 \quad .01\end{array}$

.95

$-0.19-0.21$

1.05

MCH Norms

$.15 \quad .09$

.11

.11

$-0.04-0.33$

1.15

MCH Self-efficacy

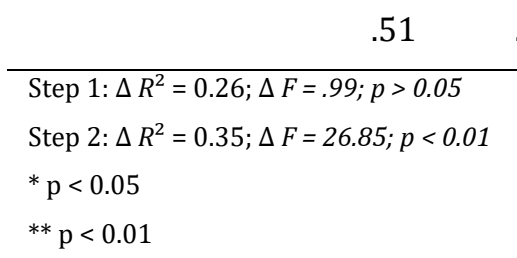

\section{Discussion}

This study examined predictors of nurses and midwives intentions to provide family planning and maternal and child healthcare services to adolescents in SA. Self-efficacy in conducting $\mathrm{MCH}$ services is the strongest positive predictor of intentions to provide MCH services to adolescents in SA. Likewise, self-efficacy in providing FP services is the strongest predictor of intentions to provide such services to adolescents. This highlights the significance of nurses' and midwives' confidence (self-efficacy) in family planning and maternal and child healthcare best practice, particularly towards adolescent FP and $\mathrm{MCH}$ needs. If the nurses and midwives in $\mathrm{MCH}$ services perceive themselves as 
incapable to provide FP and MCH best practice, the quality of their service should not be expected to be adequate.

These findings also support previous research where it is well documented that nurses and midwives typically do not feel comfortable to provide FP services, such as contraceptives to adolescents for various reasons including fear of encouraging their sexual activities [Wood et al., 2006; Ahanonu et al., 2014; Warenius et al., 2006]. Insufficient training regarding adolescent's SRH issues is among the reasons nurses and midwives are reluctant to provide FP services to adolescents [Warenius et al., 2006; Mane et al., 2015; Traore et al., 2014; Mngadi et al., 2008; Gold et al., 1997]. If nurses and midwives in MCH services in SA can be adequately skilled to attend to adolescents SRH needs, they most likely would feel confident to provide FP services to adolescents and thus increases the intentions to provide the services. This in turn, can have a positive effect on adolescents' SRH and can potentially increase the use of FP services by adolescents. Increased use of FP services by adolescents is very important in reducing the high teenage pregnancy rates in SA [Jonas et al., 2016].

These findings may also explain the poor quality of care in maternal and child healthcare facilities. Poor quality of care is now regarded as a barrier for antenatal and postnatal care, especially in low- and middle- income countries (LMICs) [Rosen et al., 2015; Naire et al., 2014; Knight et al., 2013]. Evidently, adequate utilization of FP and MCH services, particularly by adolescents relies on good quality services. A number of studies in subSaharan Africa reported poor quality of care at delivery [Rosen et al., 2015; Mselle et al., 2013; Koblinsky et al., 2006]. This is concerning as good quality best practices are necessary in $\mathrm{MCH}$ services in order to reduce maternal and child morbidity and mortality rates. Additionally, this exacerbates the situation pertaining to adolescents' sexual and reproductive healthcare (SRH) service needs as adolescents' access to and utilization of MCH services is already poor [Biddlecom et al., 2007; Glasier et al., 2006; Fishbein et al., 2010].

Nurses and midwives' FP attitudes and norms are also positively related to intentions to provide FP services to adolescents. This is in line with previous research showing that nurses and midwives attitudes towards adolescents' use of contraceptives or abortion services for example, are unfavourable [Ngomane et a., 2012; Ahanonu et al., 2014; 
Agampodi et al., 2008; Nguyeni et al., 2007]. Moreover, nurses and midwives feel if they provide adolescents with contraceptives, they are encouraging adolescent's sexual activity [Wood et al., 2006]. Clearly, the thoughts about adolescents' sexual activity being immoral affects some nurses' clinical practices, such as providing adolescents with contraceptives. These negative attitudes and persistent norms towards adolescents' sexual activities and consequently FP use and or needs pose a serious challenge when it comes to improving contraceptive use among adolescents and alleviate teenage pregnancy in SA. Contraceptive use among adolescents in SA is reported to be fairly poor [Wood et al., 2006; Jonas et al., 2016], and this finding indicates that nurses and midwives attitudes towards, and norms related to adolescents' FP services maybe associated. Efforts to increase use of FP services by adolescents should be prioritize to curb teenage pregnancy, and consequently reduce maternal and child morbidity and mortality rates.

Negative attitude towards abortion among nurses and midwives in many sub-Saharan African countries have also been reported, despite the global agreements on termination of pregnancy as a SRH right for all women and adolescents [Rehnström Loi et al., 2015; Atiya et al., 2016]. This presents a challenge in improving adolescents' use of FP services, including termination of pregnancy to prevent and or abort unwanted pregnancies. Therefore, the need to modify nurses and midwife attitudes and norms regarding adolescents' FP services is critical. Moreover, nurses and midwives attitudes towards adolescents' FP services and abortion, as well FP norms play a key role in determining their intentions to provide the services and therefore require improvement.

An important limitation in interpreting the findings of this study is that despite being based on the TPB [Ajzen et al., 1991; Motsoaledi et al., 2012], the questionnaire has not been used previously. However, the subscale analyses of the questionnaire constructs confirmed the reliability of the quantitative measurement to collect behavioral related determinants among healthcare professionals in MCH services. Further validation of these measurements in future research is needed. There is no information of participants who were not able to participate, other than their profession as nurses or midwives. However, non-participation is most likely not selective due to the study topic as the most import reason mentioned for non-participation was lack of time due to work demands. Despite this limitation, this study brings forth useful information regarding 
the psychosocial determinants of MCH best practice among nurse- midwives in SA. The cross-sectional nature of this study provides a snapshot of personal determinants of the nurses and midwives in MCH services which require improvement for adolescents FP and MCH utilization. Furthermore, the sample of nurses and midwives from different socio-economic and cultural backgrounds is in line with the general population of nurses and midwives in FP and MCH services in SA.

\section{Conclusions}

Self-efficacy is a strong positive predictor of intentions to provide both FP and MCH services. There is a need to improve and strengthen nurses' and midwives' self-efficacy in conducting both $\mathrm{MCH}$ and FP services to adolescents in SA, in order to increase intentions to provide these services. High self-efficacy and high intentions to provide FP and MCH services would increase the provision of the services and also likely to increase the utilization of services by adolescents in the country. Interventions aiming at improving self-efficacy should use behaviour change methods such as self re-evaluation and planning coping response methods [Kok et al., 2015]. This ultimately may lead to improved child health outcomes and reduction of the burden of maternal and child morbidity and mortality, as well as increased family planning utilization by adolescents in SA. The recruitment process of nurses and midwives providing SRH services also needs to be revised to further strengthen and improve adolescents' health outcomes in SA. 


\section{Submitted as}

\section{CHAPTER 5}

Jonas K, Crutzen R, Krumeich A, Roman N, van den Borne B, and Reddy P. Healthcare workers beliefs, motivation and behaviours affecting adequate provision of sexual and reproductive healthcare services to adolescents in Cape Town, South Africa. A qualitative study.

\section{Abstract}

Background: Adolescents' sexual and reproductive healthcare (SRH) needs have been prioritized globally, and they have the rights to access and utilize SRH services for their needs. However, adolescents under-utilize SRH services, especially in sub-Saharan Africa. Many factors play a role in the under-utilization of SRH services by adolescents, such as the attitude and behaviour of healthcare workers. The aim of this study therefore, was to explore and gain an in-depth understanding of healthcare workers' beliefs, motivations and behaviours affecting adequate provision of these services to adolescents in South Africa.

Methods: Twenty-four healthcare workers in public SRH services in Cape Town, South Africa participated in this qualitative study through focus group discussions. To reach the aims of this study, a total of ten focus group discussions were conducted among the SRH nurses.

Results: SRH nurses indicated that they are still experiencing challenges with termination of pregnancy due to their opposing beliefs and values. Some nurses felt that they had insufficient SRH skills, which hinder their provision of adequate SRH services to adolescents. Nurses also reported limited access to schools in the areas surrounding some clinics as a challenge for them to deliver adequate SRH services to adolescents. Conclusions: Healthcare workers are faced with numerous challenges when providing SRH services to adolescents. Providing SRH training and refresher courses to improve the nurses' skills in SRH services are required. Further research into the barriers and opportunities of the high schools' role in improving access to and utilization of SRH services by adolescents is also needed. 


\section{Introduction}

Whilst adolescents' sexual and reproductive healthcare (SRH) needs have been prioritized globally, many of these needs remain unmet [Mngadi et al., 2008; Warenius et al., 2006]. SRH services for adolescents include, services such as family planning (FP) counseling and services; prenatal and postnatal care and delivery; termination of pregnancy (TOP); post abortion care (PAC); treatment and prevention of sexually transmitted infections (STIs) including HIV; and information and counseling services regarding human sexuality [WHO, 2015]. Adolescents have the rights to fully access and utilize these services as emphasized and supported by the United Nations Population Fund Agency (UNPFA) and the World Health Organization (WHO) [WHO, 2015; UNPFA, 2014]. The availability and use of these services are essential to improve the SRH outcomes, as adolescents typically tend to engage in risky behaviours including risky sexual experiences [Hindin et al., 2009; Singh et al., 2005]. However, adolescents underutilize SRH services, especially in sub-Saharan Africa (SSA) [Mngadi et al., 2008; Warenius et al., 2006; WHO, 2015; UNPFA, 2014; Hindin et al., 2009; Singh et al., 2005] which is a contributing factor to high rates of teenage pregnancy and unsafe abortions in SA [Jonas et al., 2016a; Jonas et al., 2016b; Macleod et al., 2010; Reddy et al., 2010]. Despite supportive policies and guidelines on adolescent SRH in South Africa, the utilization is described as less than optimal [Jonas et al., 2016a; Wood et al., 2006; Geary et al., 2014; Kaufman et al., 2001].

Factors that contribute to the poor use of SRH services include the unfavourable attitude and behaviour of healthcare workers towards adolescents seeking the services [Wood et al., 2006; Geary et al., 2014; Kaufman et al., 2001; Essack et al., 2016; Atuyambe et al., 2015; Tilahun et al., 2012]. Healthcare workers' behaviour discourages young people from attending clinics and keeping follow-up appointments [Biddlecom et al., 2007; Glasier et al., 2006]. Lack of respect for adolescents' privacy and previous ill treatment by healthcare workers were some of the reasons that discouraged sexually active adolescents to seek SRH services [Biddlecom et al., 2007]. In SA there are still healthcare workers who ill-treat or refuse to assist adolescent girls who are pregnant for various reasons, including social norms regarding adolescent sexuality and their lack of skill in terms of providing some SRH services [Jonas et al., 2016b; Tsawe et al., 2014]. Thus, 
adolescents in SA are faced with difficulties accessing and utilizing SRH services because of the unfavourable behaviour of healthcare workers towards them.

Self-efficacy to provide family planning (FP) and maternal and child healthcare (MCH) services to adolescents are strongly associated with intentions to provide the services [Jonas et al., 2016b]. Furthermore the limited SRH skills and lack of confidence of some nurses in providing the services to adolescents exacerbates the problems [Jonas et al., 2016a; Jonas et al., 2016b; Kaufman et al., 2001; Tsawe et al., 2014]. Studies into the reasons for these attitudes show that the way healthcare workers conduct themselves when providing healthcare services is usually informed by their beliefs and or values [Atuyambe et al., 2015].

This implies that favorable behaviours and attitudes of healthcare workers towards adolescents seeking SRH services in SA may potentially result in a more youth-friendly service environment, which may enhance access to and utilization of the services. This in turn may help reduce the high rate of teenage pregnancies and unsafe abortions among adolescents in the country. An in-depth understanding into the determinants of health workers' unfavourable behaviours is needed to develop interventions that may contribute to an enabling environment where adolescents can fully access and utilize the SRH services. Therefore, research into the healthcare workers' behaviour and related determinants associated with providing adequate and quality SRH services to adolescents in SA is of paramount importance [Wood et al., 2006; Glasier et al., 2006; Tsawe et al., 2014].

The aim of this study was to explore and gain an in-depth understanding of professional healthcare workers' beliefs, motivations and behaviours affecting adequate provision of SRH services to adolescents in SA. The data gained will help identify where to intervene to eliminate healthcare workers' unfavourable behaviours. In addition, this data will provide ideas on how to change unfavourable behaviours and encourage adolescents to access and utilize SRH services. 


\section{Methods}

A qualitative study was conducted among healthcare workers at public clinics situated in the urban centres of Cape Town. The facilities included the Primary Health Clinics (PHCs), and the Community Health Centers (CHCs). PHCs are facilities that provide a range of primary health care services, which are usually open for eight hours or more a day based on the need of the community to be served. CHCs are facilities that usually provide more extensive services than provided by the PHCs, with 24-hour maternity, accident and emergency services and beds where health care users can be observed for a maximum of 48 hours and which normally have a procedure room but not an operating theatre [Cullinan et al., 2006]. Both PHCs and CHC provide similar services to adolescents, except in the case where the services are not being provided in PHCs. For example termination of pregnancy services are not offered in some PHCs, so these PHCs refer to CHC and other secondary levels of care for such services.

\section{Sampling}

The sampling framework for healthcare facilities consisted of public healthcare facilities that provide SRH services in the district. There are 46 clinics in Cape Town, of which 13 are the CHCs and 33 are PHCs. Not all these facilities provide SRH services, but all CHCs do. The selection of clinics was therefore based on the clinic having to provide SRH services, the community profile that the clinic is serving in order to have views from the different socio-economic statuses (SES) of the Cape Town communities, and, lastly, the feasibility to conduct the study (e.g., in terms of logistics).

Thus, facilities were selected purposively in order to meet the objectives of the study. Twelve clinics were targeted for the study, however two clinics were unsafe to be visited due to protesting in the community, which were unrelated to focus of the study and were therefore excluded. Hence, the sample consisted of ten clinics in total, of which eight have been classified as PHCs and two as CHCs, that were included in the study and all were based in the urban settings of Cape Town. The clinics were spread across the different SES settings of Cape Town, that is four clinics were in the higher SES neighbourhoods and six clinics were in the lower SES neighbourhoods. A total of ten focus group discussions (FGDs) were conducted for this study. 


\section{Participants}

A total of 28 professional healthcare workers working in SRH services were purposively approached and recruited from the clinics visited (e.g., with help from the facility managers), and were invited to participate in the different FGDs planned in their respective facilities. Of these, 24 of them agreed and participated in the study and four of them refused to participate because of limited time and patient overload in their facilities. The participants were diverse in terms educational background and cultural/religious beliefs.

This study was approved and supported by the City of Cape Town municipality under the conditions that it would not disrupt the clinic operations or disadvantage the patients. Thus, only a proportion of healthcare workers in a specific clinic were able to participate so that the clinic operations can continue with minimal distress. In some clinics where there are only four healthcare workers attending to all patients including the SRH clients, only two of them were able to participate in the FGDs as the other two had to make sure clinic operations continue. The option of after clinic operations hours was sought and proved fruitless as healthcare workers were in a hurry to go home. The FGD participants in this study expressed their views extensively and were able to elaborate where necessary because the relative small number allowed enough time for rich discussion and in-depth exploration of the different views being expressed by the participants. Therefore, due to these circumstances, including the general shortage of professional healthcare workers in public healthcare facilities in SA, and especially SRH designated healthcare workers; two to four participants in a FGD were acceptable and most optimal in terms of feasibility.

It must be noted also that healthcare workers in SRH services have no specific training or incentives to provide services to adolescents, other than the normal nursing curriculum. However, certain trainings and refresher courses are offered throughout the year for all nurses such as the refresher course on family planning guidelines, but not specific to adolescents' services.

\section{Data collection tools}

Open-ended questions were used to guide the FGD discussions and to explore and gain in-depth understanding of the beliefs, motivations and behaviours of professional 
healthcare workers in SRH services who provide the services to adolescents in SA. The belief elicitation procedure of Fishbein and Azjen (2010) was used to develop an interview guide. Appendix 1 provides an overview of statements and questions included in the interview guide. To ensure that the interview schedule was applicable and the questions were understandable to participants, two pilot interviews were conducted with healthcare professionals in SRH services, and modifications and adaptations were implemented as necessary (e.g., this statement was modified after the pilot: Healthcare workers approve of adolescents using contraceptives if they are sexually active. Probe for further details.). All FGDs were tape-recorded and hand-written notes were taken during the discussions. A brief questionnaire to document nurses' socio-demographic characteristics was also used. The ages of participants were grouped into 5 categories with a 5 -year gap in between $(1=25-30,2=31-36,3=37-42,4=43-48,5=49$ and above). Marital status was coded as ( 1 = Married, $2=$ Single, $3=$ Divorced, and $4=$ In a relationship or cohabitating). The racial categories were ( 1 = Black Africa, $2=$ Coloured, $3=$ Indian, and $4=$ White). Education level was categorised as (1 = Certificate in nursing, 2 = Diploma in nursing, and 3 = Degree in nursing). Lastly, the current position was categorised as ( 1 = Nursing sister, $2=$ Registered or enrolled nurse, and $3=$ Registered midwife).

\section{Data analysis}

Data was analyzed using thematic analysis. The data analysis followed the Tesch's eight steps for coding and analysing qualitative data, which are: 1) careful reading of all transcripts, 2) thorough examination of all FGD transcripts and making notes on the side, 3) produce a list of transpiring topics, 4) code the data using the abbreviations of the topics, 5) categorize the topics using the most descriptive wording, 6) alphabetically abbreviate each category, 7) assemble the data according to the categories, and 8) recode data if needed. The focus group discussions data was transcribed verbatim from the tape recordings, and analysed.

Typed transcripts were read several times, and codes were developed and defined based on the objectives of the study. Two independent researchers (KJ and RC) developed and checked the coding until they reached consensus. Then, codes were grouped into categories and then themes were identified. A category in this study is the "main" theme grouping the subthemes under the different questions discussed. Data was coded and 
analyzed using Nvivo qualitative data analysis software. The coded transcripts were analyzed by running query reports and primary document tables of main themes and sub-themes, to explore the issues from the various FGDs by category as shown in Table 1.

To strengthen the reliability of the data, data triangulation was conducted. Written notes and observations during data collection were performed by the research team and these were compared with the data from FGDs and corroborated the findings of this study. Furthermore, more than one person (KJ and RC) performed data analysis independently and analyses were compared. The two researchers met regularly to compare and discuss their findings until consensus was reached. All these efforts were taken to increase credibility and validity of the study findings. 
Table 1. Generating Themes

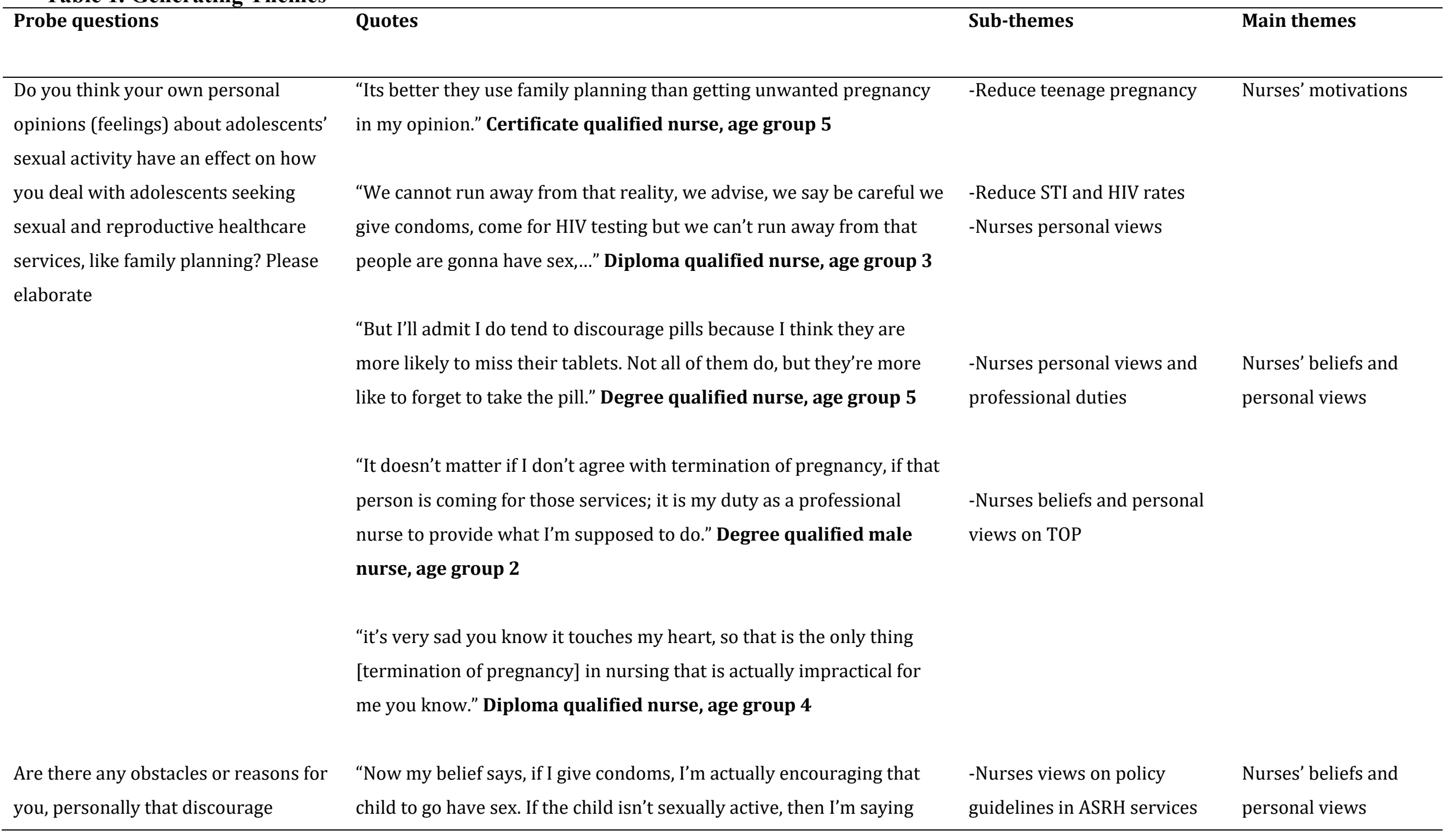


(prevent) you from providing sexual and reproductive healthcare services to adolescents? E.g. Religious or cultural beliefs?

Do you think certain

things/circumstances in the facility (obstacles) facilitate the situations where you will NOT provide SRH services? okay, take the condom and you can start." Diploma qualified nurse,

age group 3

"I do not feel comfortable dealing with teen coming for termination of

-Nurses personal views on

TOP

pregnancy, I just refer to another sister." Diploma qualified nurse,

age group 3

"I'm not pro-abortion, I feel that its murder but I will discuss that at

-Nurses personal views on

home with my family when I'm here someone walk through that door

TOP

all I see is someone who is desperate for help..." Diploma qualified

nurse, age group 2

"From my side it would be very difficult for me to give somebody a

-Nurses personal views on referral letter [for termination of pregnancy], just because I am a born again Christian." Diploma qualified nurse, age group 4

"Sometimes lack of equipment, it actually hinders our work... for

-Limited SRH resources

Challenges in SRH example, now we don't have the reproductive health form, we out of stock" Degree qualified nurse, age group 1

\section{"Shortage of staff." Diploma qualified nurse, age group 3}

"...but if anything happens in the sense that there's no staff or

-Staff shortage services

something happens, they will need me or her to help the other patients

in other sections then my patients will be sent home or my patients will 
"And its worse if you get a newly diagnosed HIV patient, you have to spend an hour with the patient, oh that one is worse, it actually gets to you... Sometimes you even leave a patient feeling like maybe I didn't do enough, but because you have to see others waiting for you and there's no time." Degree qualified nurse, age group 1

"Its just like you say next, and then next, so you don't really give attention and information. For example if they want to change the method, you don't ask why you just say ok, and just give, while if you have time you would have dealt with her better. Then you feel like 10 is overwhelming from 3-4pm and you just rush, you asking the color of discharge, when did you start experiencing the symptoms, you not even looking at her you writing because you think what's important is to treat, not think you must reiterate because you rushing. You do like rush-rush with them, because you don't have time, due to the short staff so it does happen." Degree qualified nurse, age group 4

"I have done the course [family planning course] but also I do not have the implanon training so I can't insert it so sometimes they come and want the implanon and maybe she's [the nurse who inserts implanon] - SRH skills not in so now you have to tell them there's no one to do it at that moment, so we have to give them another appointment." Diploma 
“And the patients only come after school and most schools closes at 3 pm so they come here after 3 , and there's only an hour to see more than 30 patients and we are only 3 nurses, so there is no time to do the talk." Diploma qualified nurse, age group 3

“...We are struggling to get family planning services in some high schools around the clinic, and most of the teenagers who are coming for TOP are coming from these schools we are not allowed to go to... And that shows that these schools need our FP outreach services..."

\section{Diploma qualified nurse, age group 1}

Are there any reasons for you, personally that encourage (promote) you to provide sexual and reproductive healthcare services to adolescents?

For example, what makes you more willing (motivates you) to provide sexual and reproductive healthcare services to adolescents?
"There is a lot of teenage pregnancies because I see a lot of pregnant girls and that worries me because these girls' future is ruined... she might not be able to go back to school and finish, depending on the family situations... some girls' schooling really end with their pregnancy and now she has to go find a job, any job because she is not educated...but if she was on family planning that would not happen. That is why I am happy when I see the girls on family planning, I want to help them not get pregnant." Diploma qualified nurse, age group 4

"Sometimes there are lots of babies who are dumped, the unexpected pregnancy, the problems we see in our communities, we see those

babies who are not having parents, the struggling families, the babies
-Time pressure

-School access

-Nurses concerns for

Nurses motivations

\section{-Reduce teenage pregnancy}

-Reduce unwanted pregnancy

and TOPs 
who don't have parents. This teenager that is not at school now

because she got pregnant and had to dropout." Certificate qualified

nurse, age group 5

"We actually want to decrease the number unplanned pregnancy and abortions..." Diploma qualified nurse, age group 1

"What motivates me is the rate of HIV and the rate of teenage pregnancy, and that of STIs, so that makes me want to help them, to educate them, make them understand..." Diploma qualified nurse, age group 1

“Personal experiences, you've got someone at home who's infected with HIV, your own family member got pregnant as a teenager, so you look into yourself and your experiences." Degree qualified nurse, age group 4
-Nurses concerns for

adolescents' future

-Reduce unwanted pregnancy

and TOPs

-Reduce STIs and HIV rates

-Personal experiences 


\section{Results}

\section{Participants}

Table 1 shows the demographic characteristics of the nurses who participated in the FGDs. The ages of these nurses ranged from 25 to 56 years old. Most of the women were either married or living together with their partner. Men also participated in the study, although only very few, and all of them were married. The majority of participants had a diploma in nursing and only a few had a degree in nursing. A diploma qualification is obtained through colleges and often takes up to three years, while degree is a university obtained qualification, which takes four years and includes training in extensive clinical skills. The certificate in nursing is lower than the diploma, with not much practical experience and skills of nursing care. Very few nurses in this study only had the certificate qualification of nursing. This is also reflected in their current position where a small number of them were just nursing sisters, which actually means that they are nursing assistants, as they do not have advanced nursing skills and training. A large number of them were registered nurses, and very few were specialized registered midwives (see Table 2 below). 
Table 2. Demographic characteristics of the nurses

\begin{tabular}{ll}
\hline Demographics Characteristics & $\mathbf{N}(\mathbf{\%})$ \\
\hline Gender & $3(12)$ \\
Male & $21(88 \%)$ \\
Female & \\
& \\
Age group & $4(17 \%)$ \\
1 (25-30) & $5(21 \%)$ \\
$2(31-36)$ & $4(17 \%)$ \\
$3(37-42)$ & $3(12 \%)$ \\
$4(43-48)$ & $8(33 \%)$ \\
5 (49+) & \\
Marital status & $13(54 \%)$ \\
Married & $5(21 \%)$ \\
Single & $3(13 \%)$ \\
Divorced & $2(8 \%)$ \\
In a relationship / Cohabitating & $1(4 \%)$ \\
Widow & \\
& \\
Race & $14(58 \%)$ \\
Black African & $8(34 \%)$ \\
Coloured & $1(4 \%)$ \\
Indian & $1(4 \%)$ \\
White & \\
Education & $2(8 \%)$ \\
Certificate in nursing & $13(54 \%)$ \\
Diploma in nursing & $9(38 \%)$ \\
Degree in nursing & \\
Current position & $5(21 \%)$ \\
Nursing sister & $17(71 \%)$ \\
Registered / Enrolled nurse & $2(8 \%)$ \\
Registered midwife & \\
Total & $\mathbf{2 4}(\mathbf{1 0 0 \% )}$ \\
\hline &
\end{tabular}

The results of the FGDs are presented below according to the following themes: nurses' beliefs and views of adolescents' SRH services, nurses' motivations to provide SRH services to adolescents, challenges hindering the nurses' provision of adequate SRH services to adolescents, and finally, nurses' suggestions for the improvement of access to and utilization of SRH services by adolescents. Figure 1 shows an overall picture of the themes produced during data analysis. 


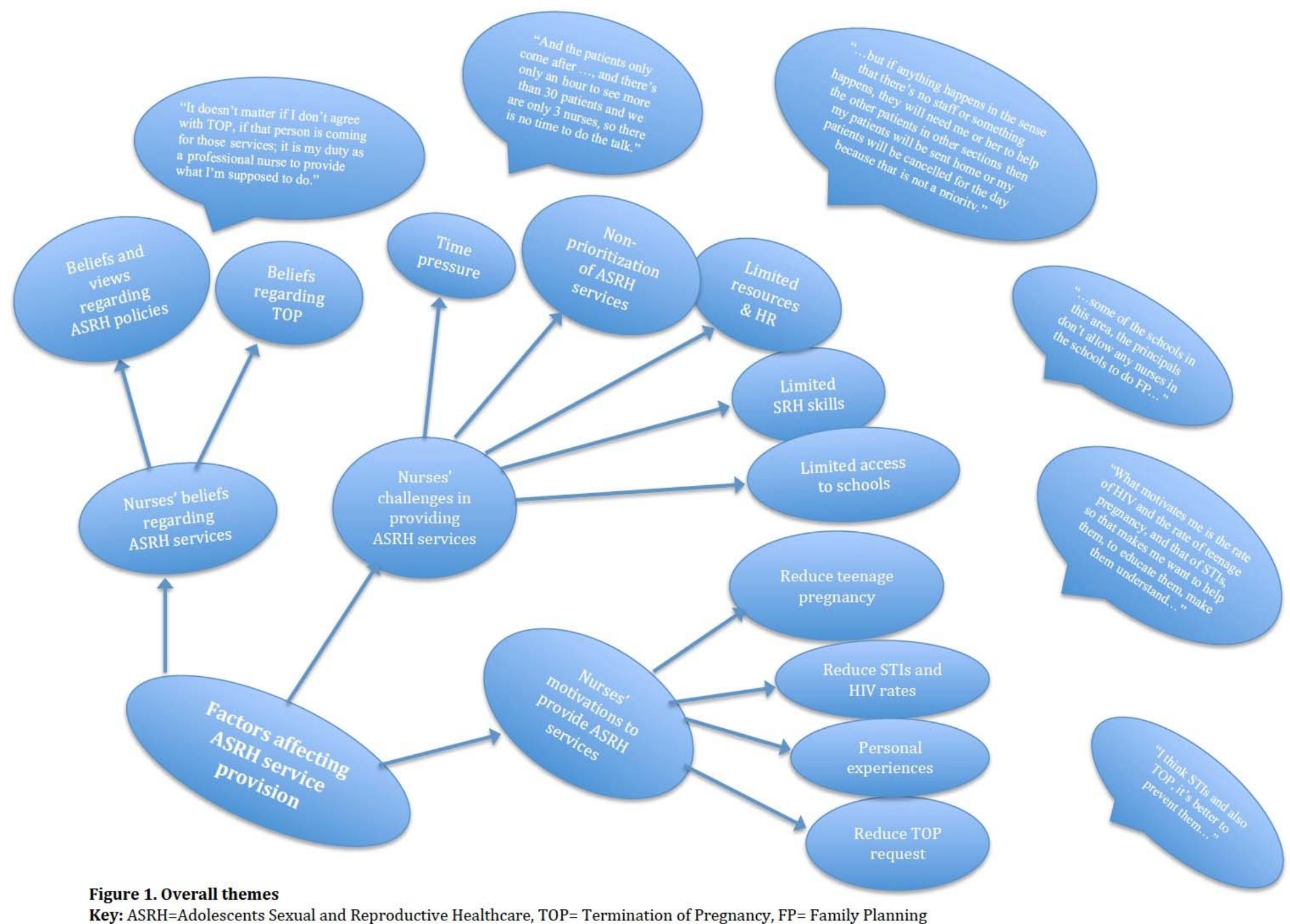




\section{Nurses' beliefs and views of adolescents' SRH services}

Discussing how the nurses view the adolescents' SRH services consequently brought up a conflict between nurses' underlying beliefs regarding the use of FP, including TOP by adolescents and their professional code. With regards to TOP, quite a few nurses had strong feelings against this basic, yet necessary component of SRH services for adolescents. The strong feelings were associated with the nurses' beliefs, which are against TOP for many nurses. However, on the other hand nurses also felt that it is their duty to provide the services irrespective of their own beliefs; otherwise they will be in conflict with their professional code of conduct. Although this was clear for many nurses, some still feel that they cannot provide this services to adolescents or even to adults because of their own beliefs, regardless of their professional responsibility to render the services required of them.

"It doesn't matter if I don't agree with termination of pregnancy, if that person is coming for those services; it is my duty as a professional nurse to provide what I'm supposed to do."

\section{Male nurse, degree qualified, age group 2}

"I'm not pro-abortion, I feel that its murder but I will discuss that at home with my family when I'm here someone walk through that door all I see is someone who is desperate for help and I have to help her as much as I can..." Diploma qualified nurse, age group 2

"No, I will never do that part [termination of pregnancy], never! I am not ready to deal with that part... my religious beliefs are against it, but also my personal beliefs." Diploma qualified nurse, age group 5

Nurses feel that the SRH services for adolescents they are expected to provide are sometimes in conflict with their own beliefs and values. According to the nurses, it appears that certain components or guidelines of the SRH services, which they have to follow when providing the services to adolescents, are contradictory to their own traditional and personal beliefs. Nurses use the provision of condoms as an example, whereby they feel providing a condom to a 12 year old is not appropriate because they think this is encouraging the teenager to start having sexual intercourse. 
"Now my belief says, if I give condoms, I'm actually encouraging that child to go have sex. If the child isn't sexually active, then I'm saying okay, take the condom and you can start." Diploma qualified nurse, age group 3

Furthermore, the nurses reported a shifted focus in the policies and guidelines they follow when serving their adolescent clients. The nurses expressed the need to reinstate the abstinence element of the prevention strategy of STI and HIV management. The nurses further stated that providing condoms for every person who comes into the clinic, according to the guidelines and policies, regardless of age and ailment they come for is somewhat out of context. The nurses gave the example of the out of context of the policies and guidelines where they found it irrelevant and inappropriate to provide a 12 year old with condoms while she specifically informed them that she is not sexually active.

“Like we don't speak about abstinence anymore, you don't even see it on posters, its not even spoken of now and its just condoms and condoms." Degree qualified nurse, age group 1

"Say you have a 12 year old who's coming for the first time for family planning, and you also offer her condoms, but she said she's not sexually active she is just trying to be safe, and now you offer condoms to her, its like you encouraging her to start having sex, you see the message you are sending now...?" Degree qualified nurse, age group 1

Nurses' motivations to provide SRH services to adolescents

In the quest to understand what motivates the nurses to provide SRH services to adolescents, the nurses were asked to share their motivations. Interesting and positive responses came up, mostly in support of better quality SRH services for adolescents. The nurses' concerns regarding the high teenage pregnancy rate among adolescents in Cape Town was a common motivation for all the nurses. The nurses also have concerns regarding the adolescents' future, which motivate them to provide the services to them so that they can finish school for example, instead of falling pregnant and ruin their future. From the FGDs, it is clear that nurses want to decrease the teenage pregnancy rate. 
"There is a lot of teenage pregnancies because I see a lot of pregnant girls and that worries me because these girls' future is ruined... she might not be able to go back to school and finish, depending on the family situations... some girls' schooling really end with their pregnancy and now she has to go find a job, any job because she is not educated...but if she was on family planning that would not happen. That is why I am happy when I see the girls on family planning, I want to help them not get pregnant." Diploma qualified nurse, age group 4

The prevention of TOP also came up from some of the FGDs. Nurses appeared to be strongly motivated to prevent unwanted pregnancies, as well as abortions among adolescents.

"We actually want to decrease the number of unplanned pregnancy and abortions..." Diploma qualified nurse, age group 1

"Lots of unwanted babies and TOPs, that makes me want to help them... I wish all of them can be on FP you know" Diploma qualified nurse, age group 1

Another important motivation for the nurses to provide SRH services to adolescents was to reduce the high rates of STIs and HIV infection among adolescents. Additionally, some nurses were inspired by their very own personal experiences as teenagers to serve SRH services to adolescents.

"What motivates me is the rate of HIV and the rate of teenage pregnancy, and that of STIS, so that makes me want to help them, to educate them, make them understand..." Diploma qualified nurse, age group 1

"Personal experiences, you've got someone at home who's infected with HIV, your own family member got pregnant as a teenager, so you look into yourself and your experiences." Degree qualified nurse, age group 4 
Challenges hindering the nurses' provision of adequate SRH services to adolescents

In this study there was also a quest on exploring what then hinders the nurses to provide adequate SRH services to adolescents. The challenges the nurses face in these services has a great potential to influence their behaviour in general, hence exploring challenges faced by the nurses was necessary. Among the many challenges nurses are faced with, time pressure was their most serious challenge in SRH services. Many of the nurses perceived the healthcare system as in favour of quantity over quality services, forgetting that different ailments need different time-allocations and this make the conditions they work under difficult to cope with.

"Its just like you say next, and then next, so you don't really give attention and information. For example if they want to change the method, you don't ask why you just say ok, and just give, while if you have time you would have dealt with her better. Then you feel like 10 is overwhelming from 3-4pm and you just rush, you asking the color of discharge, when did you start experiencing the symptoms, you not even looking at her you writing because you think what's important is to treat, not think you must reiterate because you rushing. You do like rush-rush with them, because you don't have time, due to the short staff so it does happen." Degree qualified nurse, age group 4

"And its worse if you get a newly diagnosed HIV patient, you have to spend an hour with the patient, oh that one is worse, it actually gets to you... Sometimes you even leave a patient feeling like maybe I didn't do enough, but because you have to see others waiting for you and there's no time." Degree qualified nurse, age group 1

In addition to the time pressure, nurses expressed the lack of resources as another challenge hindering the adequate provision of SRH services, together with the shortage of staff in the clinics. Furthermore, the nurses feel that the healthcare system does not prioritize the SRH needs of adolescents. When the clinics are very busy or when there is an emergency in the clinic, the nurses would be deployed to the units with high demand, leaving the SRH services not functional for that day. Moreover, the nurses expressed their limitations with certain specific SRH skills and training, such as the insertion of intra-uterine devices (IUDs) like the implanon. 
"Sometimes lack of equipment, it actually hinders our work... for example, now we don't have the reproductive health form, we out of stock " Degree qualified nurse, age group 1

“...but if anything happens in the sense that there's no staff or something happens, they will need me or her to help the other patients in other sections then my patients will be sent home or my patients will be cancelled for the day because that is not a priority." Diploma qualified nurse, age group 4

"I have done the course [FP course] but also I do not have the implanon training so I can't insert it so sometimes they come and want the implanon and maybe she's [the nurse who inserts implanon] not in so now you have to tell them there's no one to do it at that moment, so we have to give them another appointment." Diploma qualified nurse, age group 5

Nurses also reported some external factors impacting on the provision of adequate SRH services to adolescents. These factors included limited access to schools in the areas surrounding the clinic. The nurses from the clinics in the higher SES setting experienced more challenges in gaining access to the schools, while nurses from the clinics in the lower SES settings did not experience such challenges, except for one school in this setting. According to the nurses working in the higher SES settings, they find this challenge enormously frustrating for their FP outreach to adolescents.

“...We are struggling to get FP services in some high schools around the clinic, and most of the teenagers who are coming for TOP are coming from these schools we are not allowed to go to... And that shows that these schools need our FP outreach services..." Diploma qualified nurse, age group 1

Nurses' suggestions for the improvement of access to and utilization of SRH services by adolescents

After sharing their motivations, frustrations and the challenges the nurses face when providing SRH to adolescents, they had a few suggestions that they believe would make their working environment better and improve access to and utilization of these services by adolescents. The nurses in this study believed that a separate room or space for adolescents' SRH services, as well as a youth-friendly environment would improve 
access to and utilization of the services. The nurses also felt strongly that the operating clinic hours are not suitable for the adolescents and therefore needs to be extended by at least 30 minutes to an hour to accommodate the adolescents that finish school at $3 \mathrm{pm}$ and have to travel to the clinic. Some nurses recommended having some services removed from the SRH service package of adolescents, such as TOP services. Lastly, some nurses also wished to bring the services to adolescents, in their schools.

"I think if there can be a slot longer than 16:30, like maybe the clinic say we closing for youth at 17:30, just to accommodate those who are unable to come a bit earlier." Degree qualified nurse, age group 1

"If they could prevent or stop TOP at all. We really should not be having so many TOP sites and TOPs, because TOPs to me its too much they should not be so many... I would rather not have teenagers going for TOP; it should not be an option. Degree qualified nurse, age group 4

"... if the school governing body can allow us to go to the schools. Because in the clinic they come pregnant but in the schools we can do the prevention at school." Degree qualified nurse, age group 4

\section{Discussion}

This study aimed to explore beliefs, motivations and behaviours of professional healthcare workers affecting adequate provision of SRH services to adolescents. The nurses in this study seem highly motivated to serve adolescents with SRH services, although they are struggling with their personal norms and values regarding adolescents' SRH needs and services. According to the FGDs however, a combination of factors interfere with their motivation and makes it challenging for them to provide adequate quality SRH services to their adolescent clients. These factors include: their own norms and values with regards to adolescents' SRH services, the current guidelines and policies they have to follow when serving SRH services to their adolescent clients, and the healthcare systems' related factors including time constraints. All these factors play a crucial role in adequate provision of SRH services and how the nurses behave under these challenges, and that potentially affects provision of adequate SRH services 
to adolescents.

One such challenge nurses reported to have difficulties with is TOP because of their own norms and values with respect to TOP. For example, some nurses reported their religious and personal beliefs to be against TOP, which makes it difficult for them to provide this service to the adolescent in need. The nurses even went on to state that they would try to persuade adolescents to keep the baby instead of terminating the pregnancy, compromising the adolescents' rights to obtaining the services they need. Furthermore, some nurses suggested that TOP services should be totally removed from adolescents' SRH services. Negative attitudes and unfavourable behaviours of healthcare workers towards TOP have been reported elsewhere as well [Warenius et al., 2006; Rehnström Loi et al., 2015; Morris et al., 2015]. This undermines the adolescents' rights to adequate quality SRH services, including safe TOP as stipulated by the WHO and UNPFA [WHO, 2015; UNPFA, 2014]. Denying adolescents TOP not only violates their rights to SRH services, but also contributes to the high teenage pregnancy and to unsafe abortions among adolescents. It is clear that nurses in SRH services still have challenges with TOP, despite its' long existing legalization in the country. This finding is similar to other findings in sub-Saharan Africa where some nurses had negative attitudes towards TOP in general and in particular for adolescents [Mngadi et al., 2008; Rehnström Loi et al., 2015]. Healthcare workers are reported to often act as a barrier to care by failing to provide young people with supportive, nonjudgmental, youth-appropriate services [Morris et al., 2015]. Consequently, the barriers adolescents face to accessing and utilizing SRH services for their needs result in delayed care-seeking, under-utilization of services, unintended and unwanted pregnancies, and unsafe abortions [Jonas et al., 2016a; Wood et al., 2006; Geary et al., 2014; Kaufman et al., 2001; Rehnström Loi et al., 2015; Morris et al., 2015].

The nurses' challenges with TOP are coupled with their self-reported limited skills in providing SRH services such as the insertion of modern methods of FP like the implanon. SRH skills are important in delivering adequate services to adolescents. For example, low self-efficacy among nurses in conducting some SRH services to adolescents in SA has been reported, and this affects provision of adequate quality services [Jonas et al., 2016b]. Thus, the limited skills among the nurses may also act as a barrier to adolescents' access to and utilization of SRH services as they reported that some 
adolescents are turned away when the skilled nurse is not available to provide the service to them. A study in Johannesburg also reported similar findings, where several nurses reported not to be trained to insert the IUDs [Holt et al., 2012]. In order to adequately provide SRH services to adolescents, nurses in these services need to be fully trained and equipped to provide and handle all adolescent SRH issues. If the nurses possess the necessary skills in adolescents' SRH services given their motivation, it is highly likely that they may provide the required services adequately, as skills are an important predictor of service provision [Jonas et al., 2016b]. Therefore, efforts to increase training among healthcare workers in SRH to improve their SRH skills are needed. The fact that the current training and refresher courses are not mandatory and incentivised might be one of the reasons behind the limited SRH skills among the nurses and thus, needs to be addressed. Incentives may encourage and improve participation of healthcare workers in these trainings, while concurrently improving their knowledge and skills of SRH services.

Furthermore, the nurses reported time pressure which does not only affect adequate provision of SRH services to adolescents, but also affects the quality, access to, and utilization of the services by adolescents, as well as nurses' behaviour when under pressure. Because the nurses are under pressure with time, they end up rushing through the services and that often results in poor quality services. The quality of SRH services adolescents receive is an important factor in deciding whether or not to ever seek the services again, and it is unlikely that an adolescent who received poor quality service would be enthusiastic in seeking the services in future. Time pressure therefore affects the nurses' provision of quality SRH services and potentially hinders access to and utilization of the services by adolescents [Wood et al., 2006; Geary et al., 2014; Tsawe et al., 2014]. In all the FGDs, nurses reported that the operating clinic hours are not convenient for some of the school-going adolescents. Secondly, the time allocated for them on those clinics that have a youth clinic day(s) is insufficient to adequately provide quality SRH information and services to adolescents. This healthcare systems related challenge is a contributing factor to the poor access to and under-utilization of SRH services by adolescents, as they may not be able to reach the clinic during the clinic operating hours after school. This finding echoes findings from another study in SA where healthcare workers suggested extended clinic hours to accommodate schoolgoing youth who are not catered for in the "current" clinic operating hours [Holt et al., 
The reported non-prioritization of SRH services in the clinics, which substantially hinders provision of the services to some adolescents is concerning. It is worth noting that prioritization of SRH services are not the nurses' responsibilities, but that of the healthcare system itself. In addition to this non- prioritization, nurses added the shifted focus in the guidelines and policies as another healthcare system related challenge they experience. Both these healthcare systems' related challenges affect the nurses' behaviour with respect to providing adequate quality SRH services to adolescents and the adolescents' access to and utilization of SRH services. According to the nurses, whenever there's an emergency or shortage of staff in other sections of the clinic, the nurses have to turn away or cancel their adolescent clients coming for SRH services that day so that the nurses can go where there are "serious" emergency cases. This finding is a cause for concern and is in conflict with the global efforts and strategies to prioritize access to high quality SRH services for adolescents [WHO, 2015; UNPFA, 2014; Morris et al., 2015]. Moreover, some nurses reported that both the shifted focus of the guidelines and the provision of condoms to every adolescent coming for SRH services is challenging for them. Indeed, both the National contraception, fertility planning policy guidelines and the National Contraception Clinical Guidelines place an emphasis on the dual protection which is the provision of condoms in addition to the contraceptive method provided [DoH, 2012a; DoH, 2012b]. This leaves the nurses with limited room to discuss and encourage abstinence to their adolescent clients, while they appreciated the opportunity before as it enabled them to better manage their own norms and values. These findings show the complexity of the nurses' situation in SRH services, which hinders them to provide adequate quality SRH services to adolescents, as well as the barriers to accessing and utilizing SRH services by adolescents.

This study only studied nurses providing SRH services in a big urban city and not in rural areas. In rural areas, working conditions for providing the same services are substantially different from those in the big cities. Therefore, the results of this study are limited to the urban areas, and that is to take into account when interpreting its findings. A similar study would need to be undertaken in rural areas to compare our findings. Despite this limitation, the findings of this study provide sufficient information regarding the factors affecting adequate provision of sexual and reproductive healthcare 
services to adolescents in Cape Town, as well as areas of improvement in order to increase access to and utilization of the services.

To our knowledge, this study is the first study to investigate healthcare workers beliefs and behaviours in order to understand challenges affecting adequate provision of healthcare services in SA.

\section{Implications for practice}

Interventions to avert unfavourable behaviours of healthcare workers, especially towards TOP need to be intensified. Such interventions may include 'value clarification training' among the nurses in these services to eliminate negative attitudes towards TOP services at large [Holt et al., 2012]. Value clarification training might help address the nurses' beliefs by clarifying their roles as healthcare providers and how bringing their beliefs into their work may affect adequate provision of the services, leaving the clients' needs unmet. A few studies that applied this type of intervention reported positive results within the South African context, such as improved attitudes towards termination of pregnancy [Holt et al., 2012; DoH, 2012a; DoH, 2012b; Klingber'Allvin et al., 2006; Turner et al., 2008; Mitchell et al., 2005]. On a policy level, prioritization of adolescents' SRH services requires attention. Perhaps a specifically dedicated adolescent' SRH nurse, who will always be available to attend to the adolescents' SRH needs, irrespective of other emergencies in the facility, is indeed needed to meet the adolescents' SRH needs in SA. This study acknowledges the challenges the healthcare system of SA is experiencing in terms of human resources for public healthcare. However, there is a need to revisit the quantity over quality principle. The expectations that the nurses in these services assist at least 30 clients a day hinders the provision of adequate quality SRH services and information to support adolescents in making informed decisions regarding their SRH needs. Furthermore, enough time spent with adolescent during SRH consultations may help build a good rapport and consequently improve the adolescent-nurse relationship, and access to and utilization of SRH services. 


\section{Conclusion}

Professional healthcare workers are faced with numerous challenges when providing SRH services to adolescents. Provision of regular SRH training and refresher courses to improve the nurses' skills in SRH services is required. Further research into the barriers and opportunities to schools' cooperation with SRH services in order to improve access to and utilization of SRH services by adolescents are also needed. 


\section{Submitted as}

\section{CHAPTER 6}

Jonas K, Roman N, Reddy P, Krumeich A, van den Borne B, and Crutzen R. Healthcare workers' perceptions of adolescents accessing and utilizing sexual and reproductive healthcare services in Cape Town, South Africa.

\section{Abstract}

Background: In Sub-Saharan Africa (SSA) access to and utilization of sexual and reproductive healthcare (SRH) is poor. Consequently, rates of teenage pregnancy and unsafe abortions are high among adolescents in SSA, particularly in South Africa (SA). The aim of this study was to explore nurses' views on and perceptions of adolescents' barriers and needs to accessing and utilizing SRH services.

Methods: Twenty-four purposively selected healthcare workers from 10 public healthcare facilities who provide SRH services in Cape Town, South Africa participated in this qualitative study. Data was collected through 10 focus group discussions (FGDs), and audio-recorded with hand-written notes taken during the discussions. Data was analyzed using thematic analysis, following the Tesch's eight steps for coding and analysing qualitative data.

Results: SRH nurses are generally supportive of adolescents who asked for and used contraceptives. Non-compliance to family planning (FP) regimens and repeated requests of termination of pregnancies (TOPs) were perceived by nurses as behaviours which they perceive as irresponsible and are particularly frustrating to them and not in concordance with their values. The subsequent nurse-adolescent interactions sometimes appeared to hinder access to and utilization of SRH services by adolescents.

Conclusions: A good adolescent-nurse relationship may help prevent adolescents' risky behaviours, and facilitate adolescents' access to and utilization of SRH services. Nurses' counseling skills should be improved in order to effectively interact with their adolescent clients, and help improve adolescent' access to and utilization of SRH services. 


\section{Introduction}

Globally, an estimated 14 million adolescents give birth each year and more than $90 \%$ of these live births occur in low- and middle- income countries (LMICs). More than 220 million women have an unmet need for family planning (FP) in LMICs, with the majority being adolescents [Singh et al., 2012]. In Sub-Saharan Africa (SSA) access to and utilization of sexual and reproductive healthcare (SRH) is poor [Tilahun et al., 2012]. Consequently, rates of teenage pregnancy, unsafe abortions [Morris et al., 2015; Jonas et al., 2016a; Lopez et al., 2012] and sexually transmitted infections (STIs), including HIV infection rates [Tilahun et al., 2012; Morris et al., 2015] are highest among adolescents in SSA, particularly in South Africa (SA).

Adolescents' access to and utilization of SRH services, especially the use of FP services is influenced by a number of factors. For example, community reactions, societal norms, and healthcare systems' related factors all affect adolescents' access to and utilization of SRH services. Moreover, key persons in the environment of adolescents influencing adolescents' access to and utilization of SRH services are schoolteachers, healthcare workers, parents, other family members, and their own peers [Morris et al., 2015; WHO, 2014]. Adolescents' perspectives on why they under-utilize SRH, are well documented and include, for example negative attitudes and behaviour of healthcare workers [Wood et al., 2006; Tsawe et al., 2014; Macleaod et al., 2010].

Adolescents perceive healthcare workers' negative attitude and behaviour as one of the main barriers to access and utilization of SRH services. Rude, judgmental, scolding, unfriendly treatment, and lack of respect from the nurses hinder access to and utilization of SRH services, according to adolescents [Morris et al., 2015; Wood et al., 2006; Macleaod et al., 2010, Alli et al., 2013; Biddlecom et al., 2007; Hokororo et al., 2015; Geary et al., 2014]. However, there is very limited research into what reproductive healthcare nurses perceive as barriers for adolescents in accessing and utilizing SRH services. The few studies that examined nurses' perspectives on adolescents' poor access to and under-utilization of SRH services found that nurses perceive young people as ignorant of the available SRH information and services, and believe that the services are easily accessible to them [Holt et al., 2012]. Nurses reported that their limited knowledge and competency in providing SRH services to young people may hinder 
access to and utilization of services [Holt et al., 2012; Jonas et al., 2016b]. Nurses also perceived shortage of staff, age of the SRH nurse, and transport costs as other barriers to adolescents' access to and utilization of SRH [Holt et al., 2012; Jonas et al., 2016b; Herrman et al., 2013].

An insight into the nurses' perspectives on adolescents' access to and utilization of SRH might offer an explanation to their reported negative attitudes and behaviours towards adolescents seeking SRH services. For example, nurses may behave unfriendly towards adolescents seeking SRH services because of their perceptions of adolescents' sexual activity. For instance, some nurses think that providing adolescents with contraceptives would promote adolescents' sexual activity, which they use to legitimize their unfriendly treatment [Tilahun et al., 2012; Wood et al., 2006; Ahanonu et al., 2014; Nalwada et al., 2011]. This study therefore explored nurses' views on and perceptions of adolescents' barriers and needs to access and utilize SRH services. Nurses' views can complement those of adolescents and help build a bridge between the provider and the receiver, alleviating challenges on both sides for better access to and utilization of SRH services by adolescents. Understanding the nurses' perceptions of their adolescent clients and perceptions of their own behaviour will help develop interventions that aim to enhance nurses' relationship with adolescents seeking SRH services, while concurrently improving adolescents' access to and utilization of services.

\section{Methods}

A qualitative study was conducted among healthcare workers at public health clinics situated in the urban centres of Cape Town. The facilities included the Primary Health Clinics (PHCs) and Community Health Centers (CHCs). PHCs are facilities that provide a range of primary health care services. CHCs are facilities that usually provide more extensive services than provided by the PHCs, with 24-hour maternity, accident and emergency services and beds where health care users can be observed for a maximum of 48 hours and which normally have a procedure room but not an operating theatre [Cullinan et al., 2006]. 


\section{Sampling}

Public healthcare facilities that provide SRH services were selected purposively. Twelve clinics were targeted for the study, however two clinics were unsafe to be visited due to protesting in the community, which were unrelated to the focus of this study, and were therefore excluded. Hence, the sample consisted of ten clinics included in the study, of which eight have been classified as PHCs and two as CHCs. The clinics were spread across the different socio-economic status (SES) settings of Cape Town, that is four clinics were in the higher SES neighbourhoods and six clinics were in the lower SES neighbourhoods.

\section{Participants}

A total of 28 professional healthcare workers working in SRH services were purposively approached and recruited from the clinics visited (e.g., with help from the facility managers), and were invited in the different FGDs planned at their respective facilities. Of these, 24 of them agreed and participated in the study and four refused to participate because of limited time and patient overload in their facilities. Because of the shortage of professional healthcare workers in public healthcare facilities in SA generally, and especially SRH designated nurses, two to four participants in a FGD were included. Each participant received an incentive of approximately €10 (i.e., R200) in a form of a grocery voucher for his or her time. Permission to conduct the study was obtained from the University of the Western Cape Research and Ethics Committee (Ethics Reference Number: HS/16/3/49). Signed informed consent was obtained from each participant before they participated in the focus group discussons. The participants were diverse in terms educational background and cultural/religious beliefs.

This study was approved and supported by the City of Cape Town municipality under the conditions that it would not disrupt the clinic operations or disadvantage the patients. Thus, only a proportion of healthcare workers in a specific clinic were able to participate so that the clinic operations can continue with minimal distress. In some clinics where there are only four healthcare workers attending to all patients including the SRH clients, only two of them were able to participate in the FGDs as the other two had to make sure clinic operations continue. The option of after clinic operations hours was sought and proved fruitless as healthcare workers were in a hurry to go home. The FGD participants in this study expressed their views extensively and were able to 
elaborate where necessary because the relative small number allowed enough time for rich discussion and in-depth exploration of the different views being expressed by the participants. Therefore, due to these circumstances, including the general shortage of professional healthcare workers in public healthcare facilities in SA, and especially SRH designated healthcare workers; two to four participants in a FGD were acceptable and most optimal in terms of feasibility.

\section{Data collection tools}

Open-ended questions were used to guide the FGD discussions and to explore and gain in-depth understanding of the beliefs, motivations and behaviours of professional healthcare workers in SRH services who provide the services to adolescents in SA. Appendix 1 provides an overview of statements and questions included in the interview guide. To ensure that the interview guide was applicable and the questions were understandable to participants, two pilot interviews were conducted with healthcare professionals in SRH services, and modifications and adaptations were implemented as necessary (e.g., the next statement was modified after the pilot as the previous statement was not easily understandable by the nurses: Healthcare workers approve of adolescents using contraceptives if they are sexually active. Probe for further details). A total of ten focus group discussions (FGDs) were conducted for this study.

All FGDs were audio-recorded and hand-written notes were taken during the discussions, which lasted an average of 40 minutes (ranging from 30 minutes to an hour). A brief questionnaire to document nurses' socio-demographic characteristics was also used. The ages of participants were grouped into 5 categories with a 5-year gap in between $(1=25-30,2=31-36,3=37-42,4=43-48,5=49$ and above) (see Table 1 ).

\section{Data analysis}

Data was analyzed using thematic analysis. The data analysis followed the Tesch's eight steps for coding and analysing qualitative data [Tesch et al., 1990], which are: 1) careful reading of all transcripts, 2) thorough examination of all FGD transcripts and making notes on the side, 3) produce a list of transpiring topics, 4) code the data using the abbreviations of the topics, 5) categorize the topics using the most descriptive wording, 6) alphabetically abbreviate each category, 7) assemble the data according to the 
categories, and 8) recode data if needed. The focus group discussions data was transcribed verbatim from the audio recordings, and analysed.

Typed transcripts were read several times, and codes were developed and defined based on the objectives of the study. Two independent researchers (KJ and RC) developed and checked the coding until they reached consensus. Then, codes were grouped into categories and themes were identified. Data was coded and analyzed using Nvivo qualitative data analysis software. The coded transcripts were analyzed by running query reports and primary document tables of codes by theme, to explore the issues from the various FGDs by category.

To strengthen the reliability of the data, data triangulation was conducted. Written notes and observations during data collection were performed by the research team and these were compared with the data from FGDs and corroborated the findings of this study. Furthermore, more than one person (KJ and $\mathrm{RC}$ ) performed data analysis independently and analyses were compared. The two researchers met regularly to compare and discuss their findings until consensus was reached. All these efforts were taken to increase credibility and validity of the study findings.

\section{Results}

\section{Participants}

Table 1 shows the demographic characteristics of the nurses who participated in the FGDs. The majority of nurses were aged between 25 to 56 years old and were either married or living together with their partner. Men also participated in the study, although only very few, and all of them were married. The majority of participants had a diploma in nursing and only a few had a degree in nursing. A diploma qualification is obtained through colleges and often takes up to three years, while a university degree takes four years and includes training in extensive clinical skills. The certificate in nursing is lower than the diploma, with little practical experience and skills of nursing care. Very few nurses in this study only had the certificate qualification of nursing and were frequently working as nursing assistants. Most nurses were registered nurses, and very few were specialized registered midwives. 
Table 1. Demographic characteristics of the nurses

\begin{tabular}{ll}
\hline Demographics Characteristics & $\mathbf{N}(\%)$ \\
Gender & $3(12)$ \\
Male & $21(88 \%)$ \\
Female & \\
& \\
Age group & $4(17 \%)$ \\
1 (25-30) & $5(21 \%)$ \\
2 (31-36) & $4(17 \%)$ \\
3 (37-42) & $3(12 \%)$ \\
4 (43-48) & $8(33 \%)$ \\
5 (49+) & \\
Marital status & $13(54 \%)$ \\
Married & $5(21 \%)$ \\
Single & $3(13 \%)$ \\
Divorced & $2(8 \%)$ \\
In a relationship / Cohabitating & $1(4 \%)$ \\
Widow & \\
Race & $14(58 \%)$ \\
Black African & $8(34 \%)$ \\
Coloured & $1(4 \%)$ \\
Indian & $1(4 \%)$ \\
White & \\
Education & $2(8 \%)$ \\
Certificate in nursing & $13(54 \%)$ \\
Diploma in nursing & $9(38 \%)$ \\
Degree in nursing & \\
Current position & $5(21 \%)$ \\
Nursing sister & $17(71 \%)$ \\
Registered / Enrolled nurse & $2(8 \%)$ \\
Registered midwife & $\mathbf{2 4 ( 1 0 0 \% )}$ \\
Total & \\
\hline & \\
& \\
&
\end{tabular}

The results of the FGDs are presented below according to the following themes derived during data analysis: adolescents' lack of knowledge about SRH risks and services available as perceived by the nurses, adolescents are fragile and scared to access SRH services according to the nurses, and finally, adolescent' behaviours potentially influencing access to and utilization of SRH services as perceived by the nurses. 
Adolescents' lack of knowledge about SRH risks and services available as perceived by the nurses

During the FGDs, nurses were asked their views regarding challenges that they perceive adolescents to be experiencing in accessing SRH services. All nurses reported that adolescents need more information and education regarding FP services, including the different types of contraceptive methods available. Nurses perceive adolescents' limited knowledge of SRH services as a barrier and attribute it to the poor access to and underutilization of SRH services by adolescents.

"Like most of them I find that they don't have a lot of knowledge of what's happening, what SRH services are available for them, and what are the methods that we do have to offer to them." Diploma qualified nurse, age group 2

“... you try and make sure when they come here you inform them, because most of the time when they come here they come with information from friends or maybe they were referred by their sisters, so you need to make sure you give as much information as possible." Degree qualified nurse, age group 4

Nurses also believe that adolescents' knowledge is clouded with the myths around contraceptive use, which discourages them from using contraceptives. It also appears that social norms regarding the use of contraceptives exist, which prevents adolescents from accessing and utilizing the SRH services. Adolescent' sexual activity is also viewed as a taboo by some nurses.

"I can say maybe they are not aware or maybe because of myths about the other contraceptives that take long, like the implanon that takes 3 years... but they are reluctant to take modern contraceptive methods because of the myths they hear outside about these methods...." Degree qualified nurse, age group 1

"... like the implanon which is said to go around your body and eventually goes to your brain..." Diploma qualified nurse, age group 2

“...because of the stigma, if they come to the clinic it means they have boyfriends or they are sexually active." Diploma qualified nurse, age group 3 
Adolescents are fragile and scared to access SRH services according to the nurses Nurses acknowledged that adolescents are fragile and are often scared to seek SRH services, especially contraceptives. According to the nurses, they feel that adolescents are scared of what the nurses would say and how they would react to them when they come for contraceptives, or what they would find out when they reach the clinic, such as discovering that they are already pregnant or worse, infected with HIV. Thus, nurses feel that it is important for them to be friendly and sensitive when they attend to adolescents requiring contraceptives.

"They are still scared, fragile, they need more education from us, so that they know more about SRH services and other stuff they can use to protect themselves and prevent getting pregnant...So you need to be more sensitive to them and speak their language so that they can easily reach you." Male nurse, degree qualified, age group 2

"You need to be sensitive and open or warm to them when they come for FP, because they are already scared and worried what we [the nurses] going to say to them, whether they will get the service they need, and are also worried about what they would find out, 'cause some of them come here and discover that they are pregnant as we have to do the pregnancy test for first time users and those who come late for the follow-up date. And then you have to counsel the child and give some options, encourage her to talk to someone she trusts...so you have to be sensitive because now she doesn't know what to do" Degree qualified nurse, age group 2

In almost all FGDs nurses commonly reported fear of being seen in the clinic for contraceptives as a barrier for adolescents' contraceptives use. Nurses also felt that using their previous personal experiences to better relate to and communicate with adolescents is important to enable adolescents to easily reach and trust them. According to the nurses, they find that putting themselves in the adolescent' shoes and recalling their own experiences as teenagers is important when providing SRH services to adolescents, as that reminds them of how to better treat adolescents. The nurses believe that this makes it easier for adolescents to reach them and be able to ask them questions about whatever SRH issue they might have, and thus encourage them to continue to access the services for their SRH needs. 
"I went to the clinic, I think I wanted to do the FP, I think I was having the discharge as well, and the treatment I got, it was terrible, it was really terribly and I even decided on that day that one day I'm going to be a nurse and this attitude of nurses that they giving us, when I am a nurse I don't think I will be behaving or treating people like that..." Degree qualified nurse, age group 2

"As a teenager yourself, you can remember how difficult and stressful it was to go to the clinic for these things [contraceptives]... so you try and make it easy for these adolescents because you don't want them to go through what you went through, or end up being pregnant because they are afraid of you. That is how we, as SRH Sisters can help our communities reduce teenage pregnancy..." Diploma qualified nurse, age group 3

Nurses also recognize that their attitude is not always accommodating adolescents who are seeking SRH services. Nurses feel that their attitude, as well as the atmosphere at the clinic is an important factor affecting adolescents' access to and utilization of SRH services. With regards to the atmosphere at the clinic, nurses reported that the manner in which the reception desk is set up might actually be driving adolescents away as it has no privacy.

"I think we are not really accommodating, because they [adolescents] gave feedback on our outreach program saying that the staff attitude is a problem for them, so maybe that is why they don't come to this specific clinic because of the staff attitude." Diploma qualified nurse, age group 1

"And the fact that our reception is very open and then you have to speak loud if you come for something, so if you are young and you already scared, and then you have to say loudly that "I'm coming for family planning" then they rather end up not coming or just leave the clinic." Diploma qualified nurse, age group 3

In addition to the atmosphere at the clinic, nurses strongly feel that adolescents need their own physical space. Adolescents should be able to visit SRH services without being seen by family relatives or other adults or by neighbours who would most likely tell 
their parents. Nurses do not only find this as a potentially serious barrier to access to and utilization of SRH services, but also a contributing factor to FP non-compliance.

"They [adolescents] can feel free if they are on their own environment, but if there are adults, adults will judge them and they wont be free and comfortable to ask any questions they might have." Diploma qualified nurse, age group 1

"If they can have their own space, it would be nice... because they will not feel like they will see the neighbour or a friend or the Aunty you know..." Degree qualified nurse, age group 2

"Just like the other one, she came in for FP and the mother also came in for FP but she didn't know that the daughter is using FP, and they were sitting on the same line, and she said "I saw my daughter here" so now she [the daughter] will not feel comfortable to come back the next time because she knows her mother is also having the same return date." Diploma qualified nurse, age group 2

Adolescent' behaviours potentially influencing access to and utilization of SRH services as perceived by the nurses

When the nurses were asked about their perceptions of adolescents using SRH services such as contraceptives, most of them were rather ambiguous. On the one hand they feel that adolescents who are using contraceptives are responsible adolescents and they praise them for taking that decision for their SRH needs. Nurses reported that they particularly praise adolescent who returns on time for their follow-up appointments as that emphasizes the responsible behaviour of the adolescent, especially, as pointed out by some nurses, because that way unwanted pregnancies can be avoided. The nurses reported that they also encourage responsible behaviour and continue to motivate adolescents who uphold it. Encouraging and motivating responsible adolescents enhances continuous use of contraceptives by adolescents. Nurses also reported that the responsible behaviour also helps them to be more understanding and lenient when the adolescent is a couple of days late for her follow-up appointment due to some unforeseen circumstances, as the records would show that the adolescent's typical behaviour has always been responsible. 
"... when they come first time to the clinic, for me personally, I really praise them, because they have taken a good decision for themselves." Degree qualified nurse, age group 2

"And it is actually a nice thing when a 14 year old teenager comes to you and ask you for these things [contraceptives], because she knows what is right and she's responsible." Diploma qualified nurse, age group 1

"Remember that this person is starting a new thing of being on FP so you need to reenforce until she gets used to it. So its re-enforcement and as sister said, you praise where its good and you encourage them to do right all the time." Male nurse, degree qualified, age group 2

On the other hand, nurses described adolescents' irresponsible behaviours such as noncompliance with their contraceptive regimen, unsafe sex practices, "know it all" attitude, or generally not taking things and life seriously. These irresponsible behaviours particularly frustrated the nurses. Not only because it hindered them to provide adequate SRH services to adolescents, but also because this would lead to repeated requests of termination of unwanted pregnancy. Some nurses feel that some adolescents are just not grasping the seriousness and importance of using contraception to prevent unwanted pregnancy. The "know it all" attitude of adolescents hinders provision of adequate SRH information by nurses, as this attitude prevents the nurses from elaborating on some SRH information. This appears to disturb the nurses.

"They don't take things seriously. They come here for fun, they come here because the friend is on FP so I might as well be, and yet they are sexually active you know." Degree qualified nurse, age group 4

"They think they know but they don't know. They don't think about their future, they think in front of their feet, they think about their happiness, they don't think this is a delay somehow to their life, it's a fashion to them in a way [to have boyfriends]" Diploma qualified nurse, age group 5 
With regards to unsafe sex practises, nurses reported that many adolescents do not want to use condoms and as a result repeatedly come for STI management or are found positive for HIV. This unhealthy and risky behaviour did not only frustrate the nurses, but also affected them emotionally with negative consequences on how they are treating adolescents with this irresponsible behaviour. The nurses' reaction to this behaviour compromises the quality of service for the specific adolescents, and potentially threatens future access to and utilization of SRH services.

"When you give them the injection and then you give them condoms, they say "and then, what's this for now, isn't this [the contraceptive injection] for that purpose? Why must I eat a sweet in a paper?" you see they have those kind of things, at their age, its not right. Their attitude, I so wish I can just take it out from them because the way they think it's not right for them..." Male nurse, degree qualified, age group 2

"Because, I don't want to lie to you, it actually disturbs you when you get a 12 year old and you test that child positive for HIV..." Degree qualified nurse, age group 1

All nurses reported that adolescents' non-compliance with a proposed FP regimen is one of the most frustrating irresponsible behaviours of adolescents in SRH services. They explained how much they dwell on this compliance issue when adolescents come to initiate FP use. This particular behaviour also has an influence on how the nurses treat adolescents who continue to miss their follow up appointments, as they reported to use the harsh and unfriendly attitude towards the adolescent.

“...because they don't always keep their date, they come 2 weeks later, a month later saying Sister I was in Eastern Cape (EC), or Sister this and Sister that. So usually I get mad with them, because I told them the very first time they come, you must be adherent to your contraceptive, should you miss them, then you are going to get pregnant and you don't need pregnancy at this time." Degree qualified nurse, age group 2

"That shows that she's irresponsible then we must counsel her and tell her to come on the correct dates and emphasize that it is very important to prevent unwanted pregnancies." Male nurse, certificate qualified, age group 5 
"Of course I'm going to be like angry at you and my style will be different but I will give you the service and afterwards I will realise that I was blowing off steam, I need to calm down. And I will just explain this time nicely like okay this and that, please follow up on your appointments, because I'm tired now I have been telling you the same thing over and over again." Diploma qualified nurse, age group 4

Nurses also reported to be frustrated by the "repeated" requests of TOP from adolescents. Nurses perceive this behaviour as unacceptable and disturbing. They reported to have some empathy the first time an adolescent requests TOP, and use the worst-case scenarios as explanation warranting the adolescent to seek TOP, such as maybe she was raped or fears to dropout of school or university as a consequence of the pregnancy. However, when the adolescent returns the second time for TOP or the third time, the nurses reported that they actually do not feel sorry or empathise with the adolescent anymore as it shows that she is not responsible and that she is using TOP as a contraceptive method.

"But I do have a problem with the teenagers who come repeatedly for TOPs. The first time I understand and I feel for those. But when they come the second time, third time, ah. They using it as a method of contraception, and I find that very disturbing!" Degree qualified nurse, age group 5

"For the first time you think this child is young, maybe your counseling was not enough, but for the second time, you just know that they are not taking responsibility for their actions." Degree qualified nurse, age group 4

\section{Discussion}

The findings of this study show that nurses are generally supportive of adolescents using SRH services such as contraceptives and continue to encourage them to use the services. However, nurses acknowledged the challenges faced by adolescents, which prevent them from optimally accessing and utilizing SRH services. Amongst other factors, misconceptions of and myths around contraceptive use are the main challenges hindering adolescents' access to and utilization of SRH services, according to the nurses. 
This finding echoes previous research where contraceptive education for adolescents, as well as the need to address societal misconceptions and contraceptive myths have been identified as areas needing attention in order to improve adolescents' access to and utilization of SRH services [Morris et al., 2015; Lopez et al., 2012; Wood et al., 2006].

In addition to the SRH nurses who are the key persons providing contraceptive education, perhaps parents and schoolteachers such as the life orientation (LO) teachers should also provide contraceptive education to adolescents. Although it has recently been acknowledged that the LO and life skills program teachers are not necessarily following the curriculum as it is stipulated and therefore compromise the quality of this SRH education intervention [Smith et al., 2013]. More efforts to enable both teachers and parents to communicate SRH information to young people are needed to help eliminate the contraceptive misconceptions among adolescents.

Nurses particularly identified the adolescents' non-compliance issue as the main issue and they appear unfriendly in their communication with adolescent clients. Subsequently, the adolescent-nurse relationship is negatively affected as the nurses use a harsh tone and unfriendly treatment towards that particular non-compliant adolescent. Consequently, the negative adolescent-nurse relationship is likely to discourage the adolescent to come for her FP follow-up appointment and exacerbate the non-compliance behaviour, as they are afraid of further scolding. Indeed, it has been reported that the unfriendly treatment from nurses discourages youth to attend clinics for their FP follow-up appointments [Wood et al., 2006; Jewkes et al., 2009]. However, the nurses in this study do not seem to acknowledge that the scolding and "blowing off steam" caused by the late follow-up visit of adolescents is perpetuating the noncompliance. Some of the nurses believed that the scolding is necessary to show that coming late for a follow-up appointment is wrong and the scolding is meant to reiterate to the adolescent that she should not repeat such. The nurses may have good intentions emphasizing the importance of FP compliance to their adolescent clients; but how they do it might actually be perpetuating the situation of adolescents' poor access to and utilization of SRH services.

Thus, the reactions of nurses to adolescents' FP non-compliance might also act as a barrier to access and utilization of SRH services by adolescents. This may further 
increase adolescents' vulnerability to even more serious consequences such as contracting HIV infection or carry out unsafe and illegal TOP, as they may not access healthcare for their SRH needs because they fear the nurses' reactions [Wood et al., 2006; Svanemyr et al., 2015; Rehnstrom Loi et al., 2015; Mannava et al., 2015]. Therefore, improving nurses' communication skills related to SRH may greatly enhance the adolescent-nurse relationship which is essential to improve adolescents' access to and utilization of SRH services.

Repeated requests of TOPs from adolescents also bothered the nurses. It appears that some adolescents might be using TOP as a method of contraception, according to the nurses' perspectives. This is a challenge needing to be addressed. Previous studies reported nurses' strong feelings against TOP in general [Tilahun et al., 2012; Ahanonu et al., 2014; Rehnstrom Loi et al., 2015; Harries et al., 2009; Mngadi et al., 2008; Godia et al., 2013], and that might drive away adolescents after seeking TOP services, from further accessing and utilizing contraceptives services thereafter. In the previous study that we conducted we also found the same strong feelings against TOP from some nurses. Nurses reported that adolescents are often scared to seek SRH services, but when they do come for the services, some nurses are not that positive and happy to provide them with the SRH services they require, particularly TOP, according to the previous study that we conducted. It is highly likely that the nurses' reactions towards adolescents seeking TOP do not encourage the adolescent to continue seeking SRH services, such as contraceptives, or it perpetuates the non-compliance with contraceptive use of the adolescent, as the adolescent might be even more terrified to return to the clinic for her follow-up appointment post TOP services. Consequently, this may further lead to repeated requests for TOP by adolescents, as they would not come to seek contraceptives, or as a result of FP non-compliance.

Nurses in this study seem to perceive their adolescent clients as fragile and needing more information regarding their SRH services that are available to them. Nurses also acknowledged the limited knowledge of their adolescent clients regarding SRH services, as well as the need for more counseling with regards to condom use. Previous research in SSA at large, including SA, has also identified the need for more SRH information and contraceptive knowledge improvement as necessary information adolescents need to be provided with in order for them to make informed decisions regarding their SRH needs 
[Wood et al., 2006; Holt et al., 2012; Atuyambe et al., 2015; Ochako et al., 2015; ChandraMouli et al., 2014; Chandra-Mouli et al., 2013]. Furthermore, nurses emphasized the importance of being sensitive when dealing with their adolescent clients.

It is important to understand how nurses perceive their adolescent clients' needs and challenges in order to develop specific interventions that can help improve the adolescent-nurse relationship and ultimately, adolescents' access to and utilization of SRH services. For example, how the nurses react to adolescents' non-compliance as pointed above by using harsh language, needs to be improved in order not to scare away adolescents from accessing SRH services they need. Furthermore, nurses' personal feelings and values against TOP should remain personal and not affect the service provision for the adolescent in need. The nurses' resistance to TOP is potentially contributing to the high teenage pregnancy rates as some adolescents may not seek the services at all or may lead to seeking unsafe TOP services.

\section{Limitations}

This study only studied nurses providing SRH services in a big urban city and not in rural areas. Therefore, caution is to be exercised when interpreting the findings of this study as they pertain to the conditions in urban settings where culture and other traditional norms and practices may be different compared to rural settings. In rural areas, culture, religion and traditional practices are important elements shaping the community norms and values, especially with regards to adolescent sexual activities. Therefore, the results of this study are limited to the urban settings. A similar study would need to be undertaken in rural settings to compare our findings. Despite this limitation, the findings of this study provide relevant information regarding the nurses' perceptions of adolescents' needs and barriers to accessing and utilizing SRH in Cape Town, as well as areas for improvement in order to increase access to and utilization of the services.

\section{Conclusion and recommendations}

Professional healthcare workers in SRH services are enthusiastic and willing to provide SRH services to adolescents. A good adolescent-nurse relationship may help prevent adolescents' risky behaviours, as well as facilitate adolescents' access to and utilization 
of SRH services. A dedicated space for adolescents' SRH services is needed, as well as timely and proper use of FP means for adolescents need to be enhanced. Nurses' counseling skills need to be improved in order for them to effectively interact with their adolescent clients, and help improve adolescents' access to and utilization of SRH services. This counseling skills training should also include training on how to effectively communicate with adolescents in situations where their personal values are in conflict with service provision as indicated by professional standards. Improved access to and utilization of SRH services by adolescents is necessary to curb the unacceptably high rates of teenage pregnancy in the country. 


\section{GENERAL DISCUSSION}

\section{CHAPTER 7}

The primary aim of this dissertation was to identify healthcare workers' behaviours and personal determinants affecting adequate provision of sexual and reproductive healthcare (SRH) services to adolescents in South Africa (SA). The secondary aim was to uncover how healthcare workers' behaviours and personal determinants affect adolescents' access to and utilization of SRH services. This chapter provides an overview of the main findings, as well as a discussion on methodological considerations in order to inform future research. The chapter ends with policy and practical implications, and draws conclusions from the main findings.

\section{Main findings}

\section{Health care workers' behavior}

Healthcare workers in SRH services have negative behaviours (e.g., shouting and scolding at adolescents seeking SRH services) in SA, but do provide most services because of professional obligation. However, healthcare workers are not willing to provide certain SRH services to adolescents because of conflict with their own personal views and beliefs against the services. It appears that many healthcare workers still have serious issues with providing termination of pregnancy (TOP) services to an extent that they would prefer to have them removed from adolescents' SRH services.

The findings of this thesis also revealed that healthcare workers associate provision of some SRH services with promoting sexual activity among adolescents. The provision of condoms particularly is one of the services healthcare workers reported to find themselves challenged, as it implied that if they provide condoms they are encouraging adolescents to engage in sexual activity. However, not all healthcare workers in our study felt the same way about providing condoms to adolescents. Some healthcare workers acknowledged that it is a preventative measure to equip adolescents with condoms, as they do not know when adolescents are in need of protecting themselves. It is clear though that healthcare workers who feel providing condoms to adolescents is promoting sexual activity, have strong negative feelings towards providing the services to adolescents. 
Another finding concerns the circumstances in which healthcare workers particularly express negative behaviours towards adolescents seeking SRH services. Healthcare workers expressed that they especially get annoyed and irritated with adolescents who do not comply with their family planning (FP) regimen. This defaulting on FP regimen, which according to the healthcare workers many adolescents do default, leads to healthcare workers' scolding and using a harsh tone to the defaulting adolescents. This is concerning, given the fact that the consequences of this negative behavior is likely to further drive away adolescents from seeking FP services, let alone complying to the regimen.

Furthermore, some healthcare workers lack certain knowledge and skills, which are necessary for optimum quality service provision. The limited knowledge and skills to provide certain SRH is a cause for concern, as it compromises the quality of services as well as contributes to the poor access to and utilization of SRH services by adolescents. Not all healthcare workers lacked knowledge and skills in SRH services; others lacked the confidence to provide the services they have been trained to, due to limited opportunities to practice the skills. However, all healthcare workers in our study reported to lack communication skills pertaining to adolescents' SRH issues, such as the compliance issue. This limited knowledge and skills also frustrated healthcare workers and has a potential to contribute to their negative behaviours towards adolescents seeking SRH services. Consequently, the quality of services adolescents receive is below optimum standards of good quality services.

Healthcare workers' negative behaviours towards certain SRH services, such as TOP services and provision of condoms, undermine the global agreements on adolescents' access to and utilization of SRH services, which include the availability of safe and legal TOP services [WHO, 2015; UNPFA, 2013; Cohen et al., 1994]. It must be stressed that using harsh tone and scolding adolescents who defaulted on their FP regimen is not appropriate, if compliance and continued use of FP services is to be maintained. Thus, addressing healthcare workers' negative behaviours towards providing SRH services to adolescents in the country cannot be overstated. 
Healthcare workers' negative behaviours may negatively affect the provision of high quality SRH services to adolescents. Inadequate quality of SRH services may further perpetuate adolescents' poor access to and utilization of SRH services. Therefore, healthcare workers' negative behaviours might be contributing to the high rates of unwanted pregnancies, illegal and unsafe TOPs, STIs and HIV infection among adolescents in the country. Furthermore, healthcare workers' negative behaviours towards the provision of certain SRH services are a serious violation of adolescents' rights to SRH services and needs to be addressed.

The findings of this research suggest that healthcare workers' negative behaviours are influenced by a number of determinants. These determinants include, for example, traditional and religious beliefs, societal norms, perceptions of adolescents, as well as skills of healthcare workers. These determinants are discussed below.

\section{Determinants of healthcare workers' negative behaviours in SRH services}

Traditional and religious beliefs pertaining to adolescents' SRH services appear to be important determinants of negative attitudes of healthcare workers, and subsequently their behaviours. Religious and traditional beliefs mainly, but also personal views of healthcare workers regarding the provision of TOP services in particular (discussed in Chapter 5) negatively affect adequate provision of TOP services to adolescents as these beliefs lead to the negative attitudes and behaviours.

TOP services are an important and necessary component of adolescents' SRH services and therefore, adolescents have a right to access the services whenever they need it [WHO, 2015, UNPFA, 2014; Cohen et al., 1994]. Healthcare workers' negative attitudes and behaviours towards TOP services because of traditional and or religious beliefs against TOP undermine the global agreements for the availability of safe and legal TOP services for adolescents. This is a serious violation of adolescents' rights to accessing and utilizing SRH services that needs to be addressed. Similar findings on SA healthcare workers' negative attitudes towards TOP and their reluctance to provide TOP services because of religious beliefs in particular, have been reported previously [Rehnstrom Loi et al., 2015; Cooper et al., 2014; Harries et al., 2012; Wheeler et al., 2012; Harries et al., 2009; Mokgethi et al., 2006; Harrison et al., 2000]. 
The perception that provision of SRH services such as providing condoms encourages sexual intercourse is also stemmed on healthcare workers' traditional and religious beliefs against adolescents' sexual activity. Additionally, societal norms pertaining to adolescents' sexual activity also inform healthcare workers' perceptions of adolescents using SRH services. Healthcare workers often perceive adolescents seeking SRH services as irresponsible and or promiscuous, and that affects their behaviour towards them, and ultimately the quality of service adolescents receive.

Previous research on healthcare workers' perceptions of adolescents seeking SRH services also reported similar findings regarding promiscuity, and the subsequent negative attitude of healthcare workers towards adolescents accessing SRH services [Chilinda et al., 2014; Sidze et al., 2014; Tilahun et al., 2012; Mngadi et al., 2008; Warenius et al., 2006; Wood et al., 2006]. Furthermore, the poor access to and nonutilization of SRH services by adolescents has been attributed to negative attitudes of healthcare workers towards adolescents seeking SRH services and the way they communicate with adolescents by scolding and use of a harsh tone [Chilinda et al., 2014; Tilahun et al., 2012; Wood et al., 2006]. Thus, healthcare workers' perceptions of adolescents seeking SRH services, especially the perceived promiscuity hinders provision of SRH information and services and needs to be addressed in order to enhance adolescents' access to and utilization of SRH services.

Self-efficacy is another determinant influencing healthcare workers negative behaviours in SRH services. Healthcare workers in our research expressed the need for knowledge and skills' improvement. Particularly skills to provide specific SRH services such as inserting contraceptive implants and improvement of their communication skills, as elaborated in Chapters 3,4, and 5. On the one hand, some healthcare workers have the knowledge and skills or have been trained certain skills, for example how to provide basic emergency obstetric care (EmOC), but lack confidence to provide these services due to a number of reasons including limited opportunities to practice and or perform the skills (see Chapter 3). On the other hand, some healthcare workers are not fully trained to conduct EmOC services and therefore do not possess the skills and knowledge to provide it adequately, but are expected to provide it regardless (see Chapters 3,4 \& 5). For example, some healthcare workers reported to have limited knowledge of the 
mechanism of action of the emergency contraceptives (EC) and were also uncertain of the time-frame within which EC are efficacious (described in Chapter 3), but were expected to provide EC services regardless of their limited knowledge of EC. Limited knowledge and skills to provide certain SRH services among healthcare workers compromises the quality of services being provided by the inadequately skilled healthcare worker and is a cause for concern. Poor quality of services is a barrier to future access to and utilization of SRH services and therefore needs to be addressed.

These findings support previous research and echo the emphasis on improving and strengthening the skills of healthcare workers in SRH services [Ueno et al., 2015; Fawole et al., 2010]. Limited knowledge on some SRH services, such as EC and the use of partograph, among healthcare workers have also been reported in SSA at large [Mane et al., 2015; Fawole et al., 2010; Byamugisha et al., 2007; Raine et al., 2005; Gold et al., 1997]. Despite the large body of evidence on the impact of limited and or poor knowledge and skills in SRH services on access to and utilization of services, and on the quality of services [Banke-Thomas et al., 2017], efforts to improve healthcare workers' SRH knowledge and skills remain insufficient.

Insufficient supply of resources, including human resources is another determinant contributing to the negative behaviours of healthcare workers, and subsequently the inadequate provision of SRH services. Limited manpower compromises the quality of services, especially if there are lots of adolescent clients, as healthcare workers have to rush through each client with minimal attention to the quality of service being provided. Lack of resources such as essential drugs and the reproductive health forms to completely document the adolescent' reproductive health information and medical history for example, as reported in Chapters 3 and 5, also affects the quality of services. Inadequate supply of resources is likely to contribute to poor access to and utilization of SRH services. Previous studies in LMICs including SSA also showed that lack of medical supplies and human resources impede the quality of services and are barriers to care [Banke-Thomas et al., 2017; Rosen et al., 2015; Nair et al., 2014; Knight et al., 2013; Mselle et al., 2013].

Non-prioritization of SRH services within the healthcare systems also affects the behaviours of healthcare workers and significantly impedes adequate provision of 
services. On the one hand, the healthcare systems seem to have impressive and promising guidelines for adolescents' SRH services. On the other hand, however, the implementation of these guidelines is sometimes lacking or guidelines are implemented only partially. Healthcare workers' issues with the clinical guidelines, such as the disagreements with the protocols in the guidelines, may contribute to the partial implementation of SRH guidelines. Furthermore, the emergence of healthcare workers' negative behaviours may result from the disagreements with SRH guidelines, and likely to perpetuate non-prioritization of SRH services. The non-prioritization of SRH services significantly hinders access to and utilization of SRH services by young people at large.

Other healthcare systems' related factors that negatively affect healthcare workers' behaviours are patient overload, coupled with limited time and resources, which may be a consequence of the non-prioritization. This often leads to frustration and negative attitudes of healthcare workers towards the patients seeking healthcare services. If SRH services are not prioritized within the healthcare systems, provision of SRH services are unlikely to be adequate and will not meet optimum quality standards of care, which is concerning.

According to the WHO [2014] and the UNPFA [2013], non-prioritization of adolescents' SRH services is predominant in SSA, credited to the epidemic of HIV and AIDS burden. Previous research also highlighted the need to raise efforts to prioritizing SRH services for better health outcomes of adolescents [Patton et al., 2016; Greene \& Merrick, 2015; Germain et al., 2015; Chandra-Mouli et al., 2013c; WHO, 2013; Bearinger et al., 2007]. Challenges with full implementation of policy guidelines have been noted in many SSA countries [Santhya et al., 2015].

The determinants of healthcare workers' negative behaviours described above are therefore multi-level. The figure below (Figure 1) illustrates an overview of the main findings of our research at the different levels of attribution. At the organizational level, non-prioritization of SRH services, for example, influences the behaviours of healthcare workers. Also, societal norms influence behaviours of healthcare worker and the limited SRH skills of healthcare workers. Figure 1 provides an overview of these determinants at different socio-ecological levels regarding healthcare workers. 


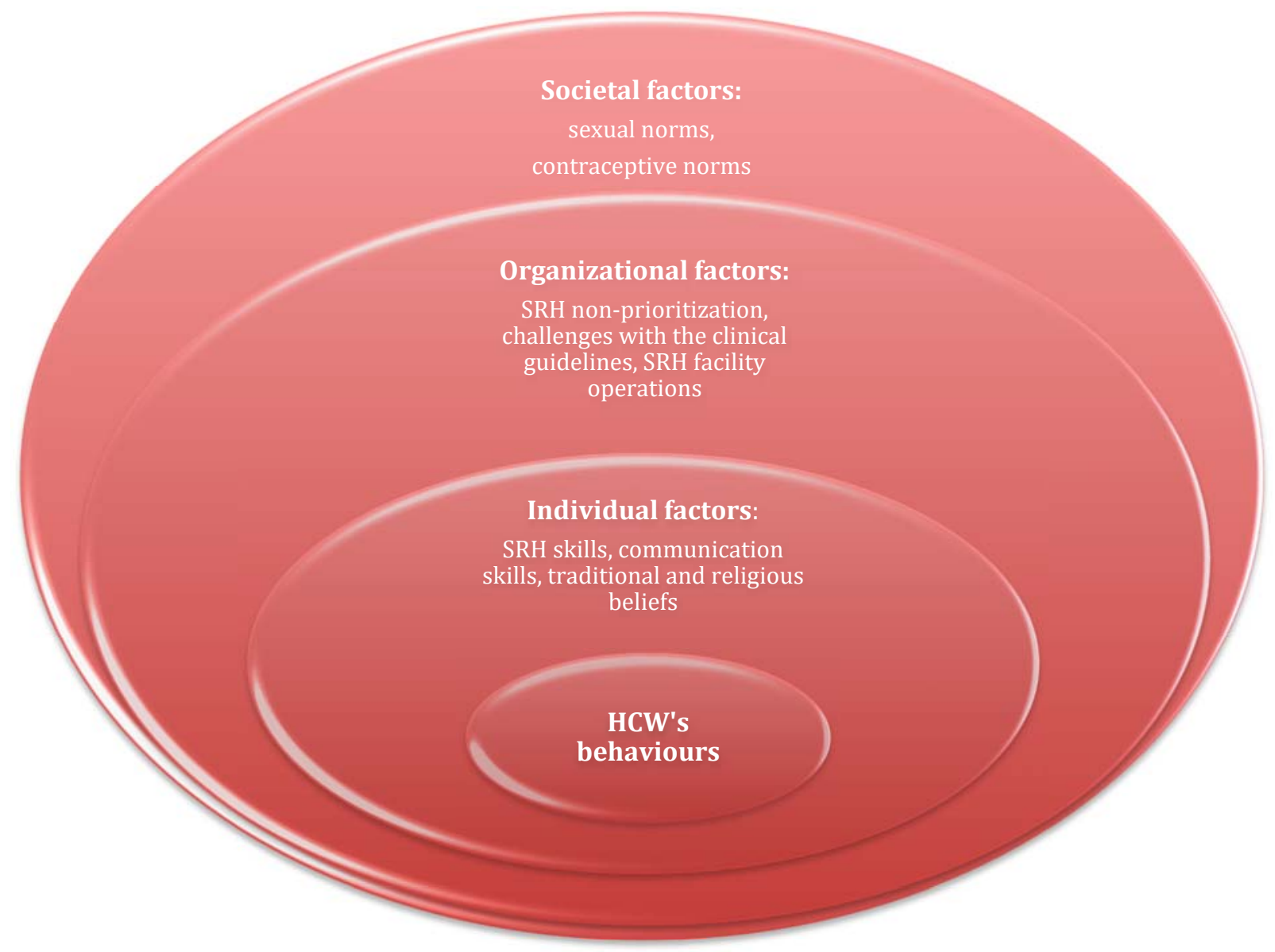

Figure 1. Overview of determinants at different socio-ecological levels.

Future considerations to change negative behaviours of healthcare workers in SRH services

The results regarding determinants of healthcare workers' negative behaviours towards adolescents' RSH services are multi-sectorial and therefore addressing these services requires a multi-level approach. Thus, future intervention efforts to change the negative behaviours of healthcare workers in SRH services and subsequently optimize quality provision of services would need to target these determinants at all levels. The Intervention Mapping (IM) approach, a theory- and evidence-based approach to systematically develop interventions, could be useful to address determinants for agents at all these levels. To influence change at the organizational level for example, the use of a participatory problem solving method, based on Organizational Development Theories (ODT) could be effective [Kok et al., 2015]. Examples of practical applications based on theoretical methods are shown in table 1 below. 
Table 1. Examples of how to change negative behaviours of healthcare workers in SRH services

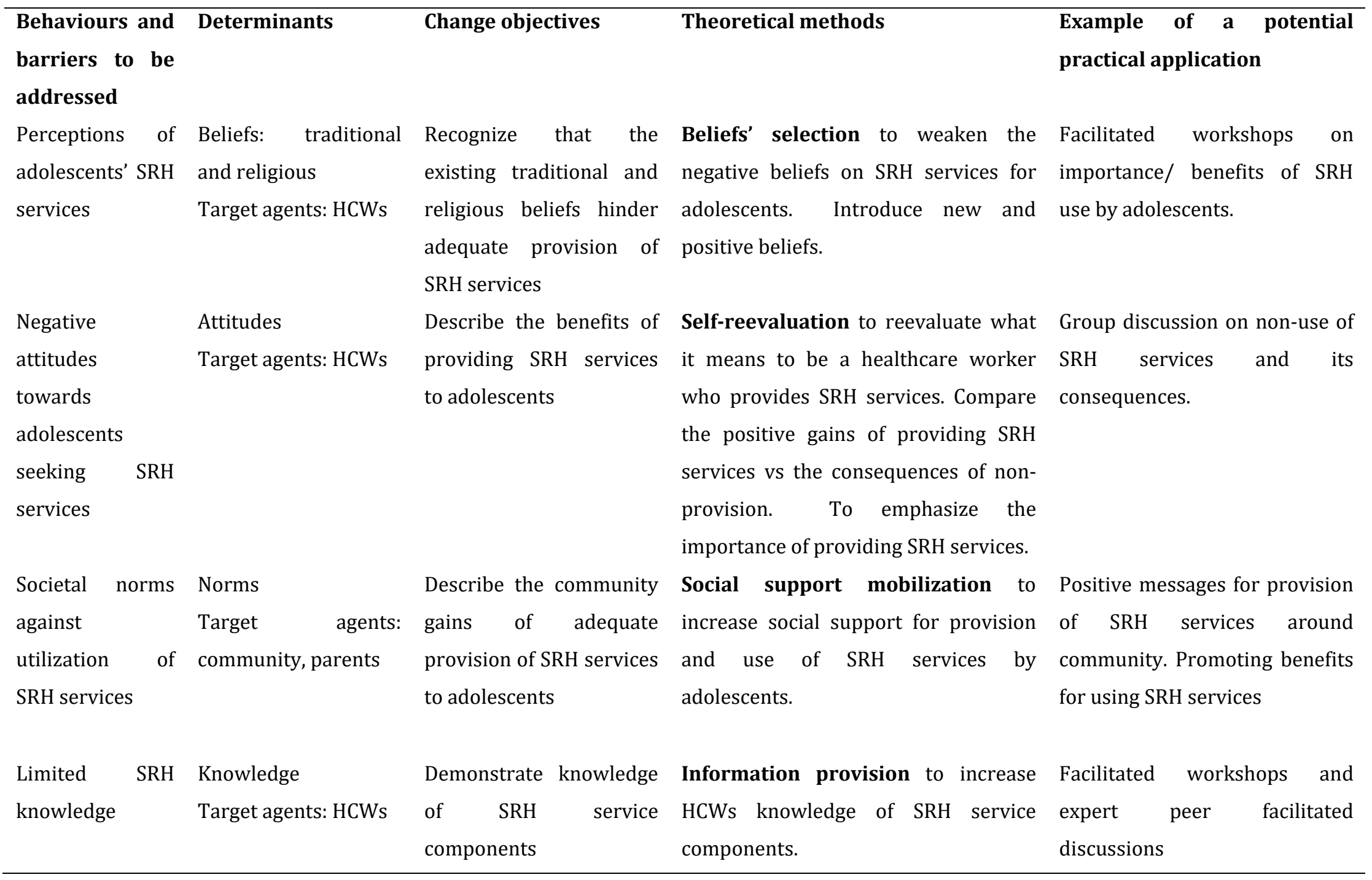




\begin{tabular}{|c|c|c|c|c|}
\hline $\begin{array}{l}\text { Limited skills to } \\
\text { provide } \quad \text { SRH } \\
\text { services }\end{array}$ & $\begin{array}{l}\text { SRH counseling and } \\
\text { communication Skills } \\
\text { Target agents: HCWs }\end{array}$ & $\begin{array}{l}\text { Demonstrate counseling } \\
\text { and communication skills } \\
\text { required during SRH } \\
\text { consultations. }\end{array}$ & $\begin{array}{l}\text { Motivational interviewing to } \\
\text { enhance collaborative communication } \\
\text { between provider and client. } \\
\text { To remove the "instructional" method } \\
\text { of communication and enforce } \\
\text { negotiation rather. }\end{array}$ & $\begin{array}{l}\text { Facilitated workshop and role- } \\
\text { plays to train HCWs to apply } \\
\text { motivational interviewing. }\end{array}$ \\
\hline $\begin{array}{l}\text { Disagreements } \\
\text { and conflicts } \\
\text { with SRH } \\
\text { guidelines }\end{array}$ & $\begin{array}{l}\text { Traditional and } \\
\text { religious beliefs } \\
\text { Target agents: HCWs, } \\
\text { facility managers, } \\
\text { policy makers/ } \\
\text { implementers }\end{array}$ & $\begin{array}{l}\text { Express importance of } \\
\text { implementing } \\
\text { components of } \text { SRH } \\
\text { guidelines }\end{array}$ & $\begin{array}{l}\text { Environmental reevaluation to } \\
\text { emphasize the consequences of poor } \\
\text { implementation of guidelines and the } \\
\text { impact it has on adolescents' SRH } \\
\text { needs and rights. }\end{array}$ & $\begin{array}{l}\text { Group discussions on poor } \\
\text { implementation of clinical } \\
\text { guidelines and reevaluation of } \\
\text { environmental barriers and } \\
\text { enablers to improve guidelines } \\
\text { full implementation }\end{array}$ \\
\hline $\begin{array}{l}\text { Limited time and } \\
\text { resources }\end{array}$ & $\begin{array}{l}\text { Limited resources } \\
\text { Target agents: } \\
\text { healthcare systems' } \\
\text { directors, facility } \\
\text { managers }\end{array}$ & $\begin{array}{l}\text { Describe the importance } \\
\text { of adequate supply of } \\
\text { resources and time }\end{array}$ & $\begin{array}{l}\text { Systems change to facilitate } \\
\text { stakeholder engagement on the } \\
\text { association between the limited } \\
\text { resources and the behaviour of } \\
\text { healthcare workers for better services } \\
\text { provision. }\end{array}$ & $\begin{array}{l}\text { Group discussions on ideas to } \\
\text { improve resources' availability } \\
\text { and to change current systems } \\
\text { operation to allow for } \\
\text { additional time for service } \\
\text { provision }\end{array}$ \\
\hline $\begin{array}{l}\text { Non- } \\
\text { prioritization of } \\
\text { SRH services }\end{array}$ & $\begin{array}{l}\text { Attitudes } \\
\text { Target agents: } \\
\text { healthcare systems' } \\
\text { directors, facility } \\
\text { managers, HCWs }\end{array}$ & $\begin{array}{l}\text { Emphasize the } \\
\text { importance of } \\
\text { prioritizing SRH services } \\
\text { and the subsequent gains } \\
\text { thereof }\end{array}$ & $\begin{array}{l}\text { Participatory problem solving from } \\
\text { high ups the ladder to down bottom to } \\
\text { enact action plan for SRH } \\
\text { prioritization of services. }\end{array}$ & $\begin{array}{l}\text { Round-table and } \begin{array}{r}\text { group } \\
\text { discussions, }\end{array} \\
\text { mechanisms, advocating for } \\
\text { SRH prioritization }\end{array}$ \\
\hline
\end{tabular}


The theoretical approaches described in table 1 are potential approaches that could be applied in developing effective interventions to change behaviours of healthcare workers in SRH services. For example, if an intervention aims to change the negative behaviours of healthcare workers towards TOP services where religious and traditional beliefs are the important determinants to be changed, then beliefs' selection might be an appropriate behaviour change method. This method is derived from the Reasoned Action Approach (RAA) and is aimed at carefully selecting the beliefs in need of change and strengthening the positive beliefs while concurrently weakening the negative beliefs, and also introducing new beliefs regarding adolescents' SRH services [Kok et al., 2015; Fishbein et al., 2010].

Mobilizing social support for positive change is an example of a method to change social norms [Kok et al., 2015]. The Sonagachi Project in India [Jana et al., 2004] is an example of an effective and sustainable multi-level intervention, which mobilized community engagement, advocacy and support resulting in lower HIV incidence among sex workers.

An example of an intervention based on theory, and combining several methods, among healthcare workers in clinical practice showed promising outcomes by modifying barriers and enhancing enablers to improve best practice [French et al., 2012]. This study applied a four-step systematic approach in identifying barriers to best practice, agents linked to the barriers, which methods could overcome the modifiable barriers and enhance the enablers, and finally how to evaluate the behaviour change [French et al., 2012]. Another study applied a multi-level approach, in which the researchers applied a multidisciplinary approach involving a two-step process for developing an implementation intervention [Curran et al., 2008]. This multi-disciplinary two-step process approach, step one being a formative (diagnostic) evaluation and step two being a multi-disciplinary team work of experts who interpret diagnostic data, showed effectiveness in primary care settings with evidence-based quality service improvements [Curran et al., 2008]. The examples in table 1 show similar approaches and combinations of several of such methods to address all change objectives for a specific target behaviour among a specific target group increase the likelihood of developing effective interventions. Thus, healthcare workers' behaviours can be improved if proper and well-designed interventions, as demonstrated in these studies, are implemented [Colquhoun et al., 2017; French et al., 2012; Curran et al., 2008]. 


\section{Methodological considerations}

This section describes the methodological approaches of the studies in this dissertation. The study designs, strengths and limitations of the approaches used are discussed below. The approaches used in this current research have implications for research and practice.

\section{Cross-sectional studies}

Two of the studies in this dissertation were cross-sectional designed studies (Chapters 2 and 4). The first study, Chapter 2 is an epidemiological overview of adolescents SRH behaviours and needed using a quantitative study applying a systematic approach among a representative sample of school going South African adolescents. The variables examined in this chapter indicated a relationship between the use of SRH services and adolescents' SRH outcomes. The study reported in Chapter 4 was a quantitative study among a sample of healthcare workers in SRH services, which investigated predictors of intentions to provide SRH services to adolescents in SA. This study showed that selfefficacy is associated with the provision of services as well as the access to and utilization of SRH services by adolescents.

Due to the cross-sectional nature of these studies however, causality could not be unequivocally established and thus caution should be exercised when interpreting the findings. It would be interesting to examine practical clinical measures in terms of skills, such as conducting observations during clinical examination of patients, auditing patient records of SRH complaints and healthcare outcomes. These would be helpful in quantifying the extent of limited knowledge and skills of healthcare workers in SRH services, as well as the quality of care being provided.

Future quantitative studies should combine both the healthcare provider and care receiver perspectives. The studies should also assess satisfaction levels of SRH clients. Clinical skills of healthcare workers must be assessed together with the determinants described in this dissertation. Additionally, analysis of facility audits may complement the findings of the individual level studies, and should be used in interpreting the findings and for the development of interventions which will effect change in SRH services. 


\section{The qualitative studies}

Chapters 5 and 6 are in-depth explorative design studies that used FGDs as means to gather data. The FGDs questions were carefully thought of and based on the belief elicitation procedure of Fishbein et al., [2010]. The findings of these two studies highlight specific areas of improvement among healthcare workers in SRH services. These areas are, healthcare workers' beliefs regarding adolescents' sexuality, including beliefs on SRH services for adolescents, self-efficacy in providing SRH services to adolescents, and other external factors such as schools involvement in SRH services. However, there are some limitations to be considered regarding these studies.

The qualitative studies were undertaken in urban settings, and thus the findings are only limited to similar settings. Many of the healthcare workers are not specialized SRH healthcare workers, for example specializing in midwifery, and are not designated youth healthcare workers. Adolescents' views with regards to their SRH services might help clarify the aspects in which healthcare workers are particularly frustrated with in SRH services as elaborated in Chapters 5 and 6. Therefore, adolescents own perspectives on the needs and barriers needs to be solicited as well in order to develop effective interventions.

The diversity in the designs of the studies described in this dissertation is the key strength of this current research. The studies were broad in nature, allowing for all possibilities in the findings. The studies were also based on existing and scientifically proven methodological approaches. For example, the use of the YRBS in Chapter 2 and the underlying theories underpinning the studies in Chapters 4, 5 and 6, such as the Theory of Planned Behaviour (TPB) and the Reasoned Action Approach (RAA). The broadness of the review study (Chapter 3) as well as its specificity in the SRH services among a wide range of healthcare workers, and yet supporting the findings of the other studies (Chapters 4, 5, and 6) is the strength of the study.

Nonetheless, the findings of all the studies included in this dissertation provide important information regarding the behaviours of healthcare workers in SRH services, as well as the impact of the behaviours on provision of adequate quality SRH services to adolescents. The combination of the approaches used in the studies described in this dissertation consolidates our findings and have provided more insight into healthcare 
workers behaviours, compared to insights that would have been derived from one approach only. With the consolidated findings of our research, we are in a better position to make recommendations for future research in a broader context, which is discussed below.

\section{Implications and recommendations}

\section{Implication for policy}

In order to increase access to and utilization of SRH services by young people, prioritization of SRH services for adolescents in particular needs to be enforced. Healthcare workers follow the current healthcare systems' policies and guidelines, but also the instructions of facility managers. Therefore, facility managers need to be advocates of adolescents SRH services through prioritizing them within their health facilities. Furthermore, policy makers need to ascertain that the policies and guidelines are actually being implemented fully in all facilities.

A dedicated SRH healthcare worker who should only provide SRH services for adolescents and will always be available for them needs to be implemented and enforced in health facilities. Facility managers, with the support of the healthcare systems and the department of health should accommodate for this in their planning and programming of adolescent SRH services. Any form of prejudice, including violation of adolescents' right to SRH services should be prohibited and a protocol to be followed when such violation occurs needs to be developed and enforced in SRH services.

Regular trainings and workshops on SRH issues, including new information, need to be incorporated to enhance healthcare workers' SRH skills. Additionally, continuous professional development (CPD) trainings need to be mandatory for all healthcare workers in SRH services. More importantly, healthcare workers who are not trained in SRH services including basic FP services should not be allowed to provide the services, as they are not equipped to do so. Only skilled healthcare workers should provide SRH services. The department of health and the association of healthcare professional should take this seriously and implement the proposed actions with immediate effect. 
Re-evaluation and revision of the quantity over quality principle by the department of health is urgently needed. Furthermore, extension of clinic operating hours, even with just one hour, would be important because it would increase access to SRH services for school going adolescents during out-of-school hours. Facility managers together with the government need to work together to negotiate the extension of operating hours. Specifically for school going adolescents, clinic operation during weekends should be considered. Investments to youth-friendly centers and or spaces for SRH services should be continued. SRH services and outreach programs should be permitted in all schools, with the cooperation of the department of education.

A revision of number of healthcare workers per health facility requires some attention, as well as, if possible, an escalation of the SRH healthcare workers in particular would be beneficial.

\section{Implications for practice}

Investments to have health facilities operating on extended clinic operating hours than currently practiced are needed. Extended clinic operating hours can be achieved, for example by letting the SRH dedicated healthcare workers to resume work a little later than the normal clinic operating hours and therefore leave later to accommodate after school services. In line with the revision of number of healthcare workers above, investments to scale up on human resources, particularly in SRH services are needed.

Interventions to improve healthcare workers behaviours towards adolescents seeking SRH services and improve quality of services are needed in order to increase access to and utilization of SRH services by adolescents in SA. The interventions should be carefully designed, taking into account all relevant factors affecting adequate provision of SRH services. Interventions based on theory- and evidence- based approaches are effective in changing behaviour in health promotion programs, for example. Table 1 provides examples of theoretical methods that could be implemented in interventions that could help improve healthcare workers behaviours and subsequently improve access to and utilization of SRH services by young people. 


\section{Conclusions}

The findings of this research provide insight into underlying reasons for healthcare workers' negative behaviours towards adolescents seeking RSH services. This is valuable to inform future intervention efforts aimed at healthcare workers and other environmental agents, which might ultimately lead to optimizing adolescents' access to and utilization of SRH in SA. First, our research reveals that healthcare workers in SRH services are battling with their own personal views and beliefs with regards to some SRH services that they have to provide to adolescents. Second, it highlights specific challenges that the healthcare workers are faced with in providing SRH services to adolescents adequately. Third, our findings reveal that healthcare workers' negative behaviours and attitudes towards adolescents seeking SRH services have a potential to act as barrier to services for adolescents. Fourth and finally, that healthcare workers' behaviours and personal determinants are influenced by a number of other factors inside and outside the healthcare systems, such as parents involvement, and the limited access to schools for delivering SRH outreach programs. The healthcare workers behaviours and personal determinants, as well the external factors all need to be taken into consideration when developing interventions which aim to improve adolescents' access to and utilization of SRH services. 


\section{References}

Accorsi S, Bilal NK, Farese P, Racalbuto V. Countdown to 2015: comparing progress towards the achievement of the health Millennium Development Goals in Ethiopia and other sub-Saharan African countries. Transactions of The Royal Society of Tropical Medicine and Hygiene. 2010;104 (5):336-42.

Agampodi SB, Agampodi TC, Piyaseeli UK. Adolescents perception of reproductive health care services in Sri Lanka. BMC health services research. 2008 May 3;8(1): 98.

Ahanonu EL. Attitudes of healthcare providers towards providing contraceptives for unmarried adolescents in Ibadan, Nigeria. Journal of family \& reproductive health. 2014;8(1): 33.

Ajzen I. The theory of planned behavior. Organizational behavior and human decision processes. 1991;50(2):179-211.

Alli F, Maharaj P, Vawda MY. Interpersonal relations between health care workers and young clients: barriers to accessing sexual and reproductive health care. Journal of community health. 2013 Feb 1;38(1):150-5.

Ameh C, Adegoke A, Hofman J, Ismail FM, Ahmed FM, \& van den Broek N. The impact of emergency obstetric care training in Somaliland, Somalia. International Journal of Gynecology \& Obstetrics. 2012. 117(3), 283-287.

Armitage CJ, Conner M. Efficacy of the theory of planned behaviour: A meta-analytic review. British journal of social psychology. 2001; 40(4):471-99.

Arnett JJ. Emerging adulthood: A theory of development from the late teens through the twenties. American psychologist. 2000 May; 55(5): 469.

Atiya KM. Maternal satisfaction regarding quality of nursing care during labor and delivery in Sulaimani teaching hospital. International Journal of Nursing and Midwifery. 
2016;8(3):18-27.

Atuyambe L, Kibira S, Bukenya J, Muhumuza C, Apolot R, \& Mulogo E. Understanding sexual and reproductive health needs of adolescents: evidence from a formative evaluation in Wakiso district, Uganda. Reproductive Health. 2015; 12(1), 35.

Bandura A. Self-efficacy. John Wiley \& Sons, Inc. New York; 1994.

Banke-Thomas OE, Banke-Thomas AO, Ameh CA. Factors influencing utilisation of maternal health services by adolescent mothers in Low-and middle-income countries: a systematic review. BMC Pregnancy and Childbirth. 2017 Feb 16;17(1):65.

Bankole A and Malarcher S. 'Removing barriers to adolescents' access to contraceptive information and services'. Studies in family planning, 2010; 41 (2), pp.117-124.

Bateman Chris. "Abortion practices undermining reformist laws"South African Medical Journal. Vol. 101, 2011, 302-304.

Bearinger LH, Sieving RE, Ferguson J, \& Sharma V. Global perspectives on the sexual and reproductive health of adolescents: patterns, prevention, and potential. The Lancet. 2007; 369(9568), 1220-1231.

Bhana D, Morrell R, Shefer T, \& Ngabaza S. South African teachers' responses to teenage pregnancy and teenage mothers in schools. Culture, health \& sexuality. 2010; 12(8), 871883.

Biddlecom AE, Munthali A, Singh S, \& Woog V. Adolescents' views of and preferences for sexual and reproductive health services in Burkina Faso, Ghana, Malawi and Uganda: original research article. African journal of reproductive health. 2007. 11(3), 99-110.

Black AY, Fleming NA, Rome ES. Pregnancy in adolescents. Adolescent medicine: State of the art reviews. 2012 Apr;23(1):123-38.

Byamugisha JK, Mirembe FM, Faxelid E, \& Gemzell-Danielsson K. Knowledge, attitudes 
and prescribing pattern of emergency contraceptives by health care workers in Kampala, Uganda. Acta obstetricia et gynecologica Scandinavica. 2007. 86(9), 1111-1116.

Cavazos-Rehg PA, Krauss MJ, Spitznagel EL, Schootman M, Cottler LB, \& Bierut LJ. Substance use and the risk for sexual intercourse with and without a history of teenage pregnancy among adolescent females. Journal of studies on alcohol and drugs. 2011; 72(2), 194.

Center for Disease Control. Conducting Trend Analysis of YRBS data. 2014. http://www.cdc.gov/healthyyouth/yrbs/pdf/yrbs_conducting_trend_analyses.pdf Viewed on 28 May 2015.

Chaibva CN, Ehlers VJ, \& Roos JH. Midwives' perceptions about adolescents' utilisation of public prenatal services in Bulawayo, Zimbabwe. Midwifery. 2010. 26(6), 16-20.

Chalmers BE, McIntyre JA, \& Meyer D. South African obstetricians' views on caesarean section. South African medical journal= Suid-Afrikaanse tydskrif vir geneeskunde. 1992. 82(3), 161-163.

Chandra-Mouli V, Greifinger R, Nwosu A, Hainsworth G, Sundaram L, Hadi S, McConville F, Benevides R, Simon C, Patkar A, Schoening E. Invest in adolescents and young people: it pays. Reproductive health. 2013 Sep 16;10(1):1.

Chandra-Mouli V, McCarraher DR, Phillips SJ, Williamson NE, Hainsworth G. Contraception for adolescents in low and middle income countries: needs, barriers, and access. Reproductive health. 2014 Jan 2;11(1):1.

Chi BH, Chansa K, Gardner MO, Sangi-Haghpeykar H, Goldenberg RL, Sinkala M, Muchimba M, \& Stringer JS. Perceptions toward HIV, HIV screening, and the use of antiretroviral medications: a survey of maternity-based health care providers in Zambia. International journal of STD \& AIDS. 2004. 15(10), 685-690.

Chigona A, \& Chetty R. Girls' education in South Africa: Special consideration to teen mothers as learners. Journal of Education for International Development. 2007; 3(1), 1-17. 
Chilinda I, Hourahane G, Pindani M, Chitsulo C, Maluwa A. Attitude of health care providers towards adolescent sexual and reproductive health services in developing countries: a systematic review. Health. 2014 Jul 28;2014.

Choice on Termination of Pregnancy, Act No. 92, Nov. 12 Art 2b. www.gov.za/sites/www.gov.za/files/Act92of1996.pdf

Accessed 28 November 2016.

Choice on Termination of Pregnancy Amendment Act No 1 of 2008. http://www.gov.za/sites/www.gov.za/files/a1-08.pdf

Accessed 28 November 2016.

Cohen SA, Richards CL. The Cairo consensus: population, development and women. Family planning perspectives. 1994 Nov 1;26(6):272-7.

Colquhoun HL, Squires JE, Kolehmainen N, Fraser C, Grimshaw JM. Methods for designing interventions to change healthcare professionals' behaviour: a systematic review. Implementation Science. 2017 Mar 4;12(1):30.

Conde-Agudelo A, Belizán JM, Lammers C. Maternal-perinatal morbidity and mortality associated with adolescent pregnancy in Latin America: Cross-sectional study. American journal of obstetrics and gynecology. 2005 Feb 28;192(2):342-9.

Cooper, Diane; Strebel, Anna; Colvin, Christopher "Conscientious Objection and its impact on abortion service provision in South Africa: A qualitative study". Reproductive Health. Vol. 11, part 16, 2014, p. 1-7

Cullinan K. Health Services in South Africa. 2006.

https://www.health-e.org.za/wp-

content/uploads/2013/04/Health services briefing doc.pdf.

Accessed November 16, 2016 
Curran GM, Mukherjee S, Allee E, Owen RR. A process for developing an implementation intervention: QUERI Series. Implement Sci. 2008;3(1):17.

Davis D, O'Brien MAT, Freemantle N, Wolf FM, Mazmanian P, \& Taylor-Vaisey A. Impact of formal continuing medical education: do conferences, workshops, rounds, and other traditional continuing education activities change physician behavior or health care outcomes?. Jama. 1999; 282(9), 867-874.

Department of Health. Guidelines for Maternity Care in South Africa: A Manual for Clinics, Community Health Care Centres and District Hospitals. 2015

Department of Health, South Africa. Guidelines: National contraception, fertility planning policy. A companion of the National Contraception Clinical Guidelines. 2012a. Pretoria

Department of Health, South Africa. National Contraception Clinical Guidelines. 2012b. Pretoria

Department of Health. Saving mothers: second report on the Confidential Enquiries into Maternal Deaths in South Africa 1999-2001. Pretoria: Department of Health; 2002.

Eaton L, Flisher AJ, \& Aarø LE. Unsafe sexual behaviour in South African youth. Social science \& medicine. 2003; 56(1), 149-165.

Ebrahim SH, \& Gfroerer J. Pregnancy-Related Substance Use in the United States During 1996-1998. Obstetrics \& Gynecology. 2003; 101(2), 374-379.

Ehiri JE, Oyo-Ita AE, Anyanwu EC, Meremikwu MM, \& Ikpeme MB. Quality of child health services in primary health care facilities in south-east Nigeria. Child: care, health and development. 2005. 31(2), 181-191.

Ersdal HL, Verkuyl DA, Björklund K, \& Bergström S. Symphysiotomy in Zimbabwe; postoperative outcome, width of the symphysis joint, and knowledge, attitudes and practice among doctors and midwives. PloS one. 2008. 3(10), e3317. 
Essack Z, Toohey J, Strode A. Reflecting on adolescents' evolving sexual and reproductive health rights: canvassing the opinion of social workers in KwaZulu-Natal, South Africa. Reproductive Health Matters. 2016. 24(47): 195-204.

Evens E, Otieno-Masaba R, Eichleay M, McCARRAHER DONNA, Hainsworth G, Lane C, Makumi M, \& Onduso P. Post-abortion care services for youth and adult clients in Kenya: a comparison of services, client satisfaction and provider attitudes. Journal of biosocial science. 2014. 46(01), 1-15.

Fawole AO, Adekanle DA, \& Hunyinbo KI. Utilization of the partograph in primary health care facilities in southwestern Nigeria. Nigerian journal of clinical practice. 2010. 13(2).

Fawole AO, Hunyinbo KI, \& Adekanle DA. Knowledge and Utilization of the Partograph among obstetric caregivers in South West Nigeria. African journal of reproductive health. 2009. 12(1), 22-29.

Field A. Discovering statistics using SPSS. Sage publications. 2009.

Fishbein M, Ajzen I. Predicting and changing behavior: The reasoned action approach. Taylor \& Francis; 2010 May 20.

French SD, Green SE, O'Connor DA, McKenzie JE, Francis JJ, Michie S, Buchbinder R, Schattner P, Spike N, Grimshaw JM. Developing theory informed behaviour change interventions to implement evidence into practice: a systematic approach using the Theoretical Domains Framework. Implement Sci. 2012;7(1):38.

Ganchimeg E, Ota N, Morisaki M, Laopaiboon P, Lumbiganon J, Zhang B, et al. Pregnancy and childbirth outcomes among adolescent mothers: a World Health Organization multicountry study. BJOG. 2014; 121(1):40.

Ganle JK, Parker M, Fitzpatrick R, Otupiri E. A qualitative study of health system barriers to accessibility and utilization of maternal and newborn healthcare services in Ghana after user-fee abolition. BMC pregnancy and childbirth. 2014 Dec 21;14(1):425. 
Geary RS, Gómez-Olivé FX, Kahn K, Tollman S, Norris SA. Barriers to and facilitators of the provision of a youth-friendly health services programme in rural South Africa. BMC health services research. 2014. 14(1):1.

Germain A. (2014). Promoting healthy adolescent development through comprehensive sexuality education. Global Public Health, 10(2). doi:10.1080/17441692.2014.986176

Glasier A, Gulmezoglu AM, Schmid GP, Moreno CG, Van Look PF. Sexual and reproductive health: a matter of life and death. Lancet. 2006. 368: 1595-607.

Godia PM, Olenja JM, Lavussa JA, Quinney D, Hofman JJ, Van Den Broek N. Sexual reproductive health service provision to young people in Kenya; health service providers' experiences. BMC health services research. 2013 Nov 14;13(1):1.

Gold MA, Schein A, \& Coupey SM. Emergency contraception: a national survey of adolescent health experts. Family planning perspectives. 1997. 15-19.

Grant MJ, \& Hallman KK. Pregnancy-related School Dropout and Prior School Performance in KwaZulu-Natal, South Africa. Studies in Family Planning. 2008; 39(4), 369-382.

Greene M and Merrick T, 2015. The Case for Investing in Research to Increase Access to and Use of Contraception among Adolescents. Seattle, WA: Alliance for Reproductive, Maternal, and Newborn Health.

Guttmacher Institute: Althaus FA. Work in progress: the expansion of access to abortion services in South Africa following legalization. International Family Planning Perspectives. 2000. 26(2):84. http://www.guttmacher.org/pubs/journals/2608400.html Accessed 13 December 2016.

Haile-Mariam A, Tesfaye N, Otterness C, \& Bailey PE. Assessing the health system's capacity to conduct neonatal resuscitation in Ethiopia. Ethiopian medical journal. 2012. $50(1), 43-55$.

Haroz AE. South Africa's 1996 Choice on Termination of Pregnancy Act: expanding choice 
and international human rights to black South African women. Vand. J. Transnat'l L.. 1997;30:863.

Harries J, Cooper D, Strebel A, Colvin CJ. Conscientious objection and its impact on abortion service provision in South Africa: a qualitative study. Reproductive health. 2014 Feb 26;11(1):16.

Harris LH. Recognizing conscience in abortion provision. New England Journal of Medicine. 2012 Sep 13;367(11):981-3.

Harries J. "What health-care providers say on providing abortion care in Cape Town South Africa: findings from a qualitative study." (2010).

Harries J, Stinson K, Orner P. Health care providers' attitudes towards termination of pregnancy: A qualitative study in South Africa. BMC Public Health. 2009 Aug 18;9(1):1.

Harrison A, Newell M-L, Imrie J and Hoddinott G. HIV prevention for South African youth: which interventions work? A systematic review of current evidence. BMC Public Health, $2010 ; 10(1)$, p.1.

Hendriksen ES, Pettifor A, Lee SJ, Coates TJ, \& Rees HV. Predictors of condom use among young adults in South Africa: The reproductive health and HIV research unit national youth survey. American Journal of Public Health. 2007; 97(7), 1241.

Herrman J. The provision of reproductive health services by school-based health centers: Policy Implications. In42nd Biennial Convention (16 November-20 November 2013) 2013 Nov 18. STTI.

Hindin MJ, \& Fatusi AO. Adolescent sexual and reproductive health in developing countries: an overview of trends and interventions. International perspectives on sexual and reproductive health. 2009. 58-62.

Holmberg L, \& Berg-Kelly K. Health, health-compromising behaviour, sexuality and involvement in pregnancy among 18-year-old Swedish males: a cross-sectional survey. 
Acta Paediatrica. 2002; 91(7), 838-843.

Hokororo A, Kihunrwa AF, Kalluvya S, Changalucha J, Fitzgerald DW, Downs JA. Barriers to access reproductive health care for pregnant adolescent girls: a qualitative study in Tanzania. Acta Paediatrica. 2015 Dec 1;104(12):1291-7.

Holt K, Lince N, Hargey A, Struthers H, Nkala B, McIntyre J, Gray G, Mnyani C, Blanchard K. Assessment of service availability and health care workers' opinions about young women's sexual and reproductive health in Soweto, South Africa. African journal of reproductive health. 2012. 16(2): 283-94.

Hussein J, Bell J, Nazzar A, Abbey M, Adjei S, \& Graham W. The skilled attendance index: proposal for a new measure of skilled attendance at delivery. Reproductive health matters. 2004. 12(24), 160-170.

Ijadunola KT, Ijadunola MY, Esimai OA, \& Abiona TC. New paradigm old thinking: the case for emergency obstetric care in the prevention of maternal mortality in Nigeria. BMC women's health. 2010.10(1), 1.

Imamura M, Tucker J, Hannaford P, Da Silva MO, Astin M, Wyness L, et al. Factors associated with teenage pregnancy in the European Union countries: a systematic review. The European Journal of Public Health. 2007; 17(6), 630-636.

Jana S, Basu I, Rotheram-Borus MJ, Newman PA. The Sonagachi Project: a sustainable community intervention program. AIDS Education and Prevention. 2004 Oct 1;16(5):40514.

Jewkes R, Morrell R, \& Christofides N. Empowering teenagers to prevent pregnancy: lessons from South Africa. Culture, Health \& Sexuality. 2009; 11(7), 675-688.

Jewkes R, Vundule C, Maforah F, \& Jordaan E. Relationship dynamics and teenage pregnancy in South Africa. Social Science \& Medicine. 2001; 52(5), 733-744.

Jonas K, Crutzen R, van den Borne B, Sewpaul R, Reddy P. Teenage pregnancy rates and 
associations with other health risk behaviours: a three-wave cross-sectional study among South African school-going adolescents. BMC Reproductive Health. 2016a. 13(1): 50. DOI: 10.1186/s12978-016-0170-8

Jonas K, Crutzen R, van den Borne B, \& Reddy P. Healthcare workers' behavior and personal determinants associated with providing sexual and reproductive healthcare services to teenagers in sub-Saharan Africa: a systematic review. PROSPERO 2015. CRD42015017509 Available

from http://www.crd.york.ac.uk/PROSPERO REBRANDING/display record.asp?ID=CRD42015 $\underline{017509}$

Jonas K, Reddy P, van den Borne B, Sewpaul R, Nyembezi A, Naidoo P, Crutzen R. Predictors of nurses' and midwives' intentions to provide maternal and child and healthcare services to adolescents in South Africa. BMC Health Services Research. 2016b. 16:658. DOI: 10.1186/s12913-016-1901-9

Kaufman CE, de Wet T, Stadler J. Adolescent pregnancy and parenthood in South Africa. Stud Fam Plann. 2001. 32: 147-60.

Keeney GB, Cassata L, \& McElmurry BJ. Adolescent health and development in nursing and midwifery education. WHO, 2004.

Kimberly HH, Murray A, Mennicke M, Liteplo A, Lew J, Bohan JS, Tyer-Viola L, Ahn R, Burke T, \& Noble VE. Focused maternal ultrasound by midwives in rural Zambia. Ultrasound in medicine \& biology. 2010. 36(8), 1267-1272.

Kirby, D. Understanding what works and what doesn't in reducing adolescent sexual risktaking. Family Planning Perspectives. 2001; 33, 1-14.

Klingberg-Allvin M, Nga NT, Ransjö-Arvidson AB, Johansson A. Perspectives of midwives and doctors on adolescent sexuality and abortion care in Vietnam. Scandinavian journal of public health. 2006. 34(4): 414-21.

Knight HE, Self A, Kennedy SH. Why are women dying when they reach hospital on time? 
A systematic review of the 'third delay'. PloS one. 2013; 8(5): e63846.

Koblinsky M, Matthews Z, Hussein J, Mavalankar D, Mridha MK, Anwar I, Achadi E, Adjei S, Padmanabhan P, van Lerberghe W, Lancet Maternal Survival Series steering group. Going to scale with professional skilled care. The Lancet. 2006;368 (9544):1377-86.

Kok G, Gottlieb NH, Peters GJ, Mullen PD, Parcel GS, Ruiter RA, Fernández ME, Markham C, Bartholomew LK. A taxonomy of behaviour change methods: an intervention mapping approach. Health psychology review. 2015;19:1-6. http://dx.doi.org/10.1080/17437199.2015.1077155

Kuruvilla S, Bustreo F, Kuo T, Mishra CK, Taylor K, Fogstad H, Gupta GR, Gilmore K, Temmerman M, Thomas J, Rasanathan K. The Global strategy for women's, children's and adolescents' health (2016-2030): a roadmap based on evidence and country experience. Bull World Health Organ. 2016. 94(5):398-400.

Lawani LO, Eze JN, Anozie OB, Iyoke CA, \& Ekem NN. Obstetric analgesia for vaginal birth in contemporary obstetrics: a survey of the practice of obstetricians in Nigeria. BMC pregnancy and childbirth. 2014; 14(1), 1.

León FR, Lundgren R, \& Jennings V. Provider selection of evidence-based contraception guidelines in service provision: A study in India, Peru, and Rwanda. Evaluation \& the health professions. 2007.

Lopez LM, Hiller JE, Grimes DA. Education for contraceptive use by women after childbirth. Cochrane Database Syst Rev 2012;8: CD001863.

Macleod CI, Tracey T. A decade later: follow-up review of South African research on the consequences of and contributory factors in teen-aged pregnancy. South African Journal of Psychology. 2010. 40(1): 18-31.

Macleod C. The 'causes' of teenage pregnancy: Review of South African research--Part 2. South African Journal of Psychology. 1999; 29(1), 8. 
Makiwane M, Udjo E, Richter L, \& Desmond C. Is the Child Support Grant associated with an increase in teenage fertility in South Africa? Evidence from national surveys and administrative data. HSRC, Pretoria. 2006.

Mamabolo LR, Tjallinks JE. Experiences of registered nurses at one community health centre near Pretoria providing termination of pregnancy services. Africa Journal of Nursing and Midwifery. 2010 Jan 1;12(1):73-86.

Mané B, Brady M, Ramarao S, \& Thiam A. Emergency contraception in Senegal: Challenges and opportunities. The European Journal of Contraception \& Reproductive Health Care. 2015. 20(1), 64-73.

Mannava P, Durrant K, Fisher J, Chersich M, Luchters S. Attitudes and behaviours of maternal health care providers in interactions with clients: a systematic review. Globalization and health. 2015.11(1):36.

Manzini N. Sexualinitiation andchildbearing among adolescent girls in KwaZulu Natal, South Africa. Reproductive Health Matters. 2001; 9(17).

Maramagi CA, Lubanga RG, Kiguli S, Ekwaru PJ, \& Heggenhougen K. Health providers' counselling of caregivers in the Integrated Management of Childhood Illness (IMCI) programme in Uganda. African health sciences. 2004. 4(1), 31-39.

Martin JA, Hamilton BE, Ventura SJ, Osterman MJ, Wilson EC, Mathews TJ. Births: final data for 2010. National vital statistics reports. 2012 Aug 28; 61(1):1-72.

McAuliffe E, Daly M, Kamwendo F, Masanja H, Sidat M, \& de Pinho H. The critical role of supervision in retaining staff in obstetric services: a three country study. PloS one. 2013. 8(3), e58415.

Mchunu G, Peltzer K, Tutshana B, \& Seutlwadi L. Adolescent pregnancy and associated factors in South African youth. African health sciences. 2013; 12(4), 426-434.

Mirkuzie AH, Sisay MM, Reta AT, \& Bedane MM. Current evidence on basic emergency 
obstetric and newborn care services in Addis Ababa, Ethiopia; a cross sectional study. BMC pregnancy and childbirth. 2014. 14(1), 1.

Mkhwanazi N. Understanding teenage pregnancy in a post-apartheid South African township. Culture, health \& sexuality. 2010; 12(4), 347-358.

Mitchell EM, Trueman K, Gabriel M, Bock LB. Building alliances from ambivalence: evaluation of abortion values clarification workshops with stakeholders in South Africa. African journal of reproductive health. 9(3): 89-99.

Mngadi PT, Faxelid E, Zwane IT, Höjer B, \& Ransjo-Arvidson AB. Health providers' perceptions of adolescent sexual and reproductive health care in Swaziland. International nursing review. 2008. 55(2), 148-155.

Mokgethi NE, Ehlers VJ, Van der Merwe MM. Professional nurses' attitudes towards providing termination of pregnancy services in a tertiary hospital in the North West province of South Africa. Curationis. 2006 Sep 28;29(1):32-9.

Morris JL, Rushwan H. Adolescent sexual and reproductive health: The global challenges. International Journal of Gynecology \& Obstetrics. 2015. 131: S40-2.

Motsoaledi AP. National Contraception and Fertility Planning Policy and Service Delivery Guidelines A companion to the National Contraception Clinical Guidelines. 2012, SA.

Moyer CA, Mustafa A. Drivers and deterrents of facility delivery in sub-Saharan Africa: a systematic review. Reproductive health. 2013 Aug 20;10(1):40.

Mselle LT, Moland KM, Mvungi A, Evjen-Olsen B, Kohi TW. Why give birth in health facility? Users' and providers' accounts of poor quality of birth care in Tanzania. BMC health services research. 2013;13 (1):174.

Nair M, Yoshida S, Lambrechts T, Boschi-Pinto C, Bose K, Mason EM, Mathai M. Facilitators and barriers to quality of care in maternal, newborn and child health: a global situational analysis through metareview. BMJ open. 2014; 4(5):e004749. 
Nalwadda G, Mirembe F, Tumwesigye NM, Byamugisha J, Faxelid E. Constraints and prospects for contraceptive service provision to young people in Uganda: providers' perspectives. BMC health services research. 2011 Sep 17;11(1):1.

National Department of Social Development (DSD), South Africa. National Adolescent Sexual and Reproductive Health and Rights Framework Strategy. 2015. http://www.dsd.gov.za/index2.php?option=com docman\&task=doc view\&gi $\underline{\mathrm{d}=578 \& \text { Itemid }=3}$ Accessed 28 November 2016.

Neal SE, Chandra-Mouli V, Chou D. Adolescent first births in East Africa: disaggregating characteristics, trends and determinants. Reprod Health. 2015;12 (13):13.

Neal S, Matthews Z, Frost M, Fogstad H, Camacho AV, \& Laski L. Childbearing in adolescents aged 12-15 years in low resource countries: a neglected issue. New estimates from demographic and household surveys in 42 countries. Acta Obstetricia et Gynecologica Scandinavica. 2012; 91(9), 1114-1118. doi: 10.1111/j.16000412.2012.01467.x

Ndikom CM, \& Onibokun A. Knowledge and behaviour of nurse/midwives in the prevention of vertical transmission of HIV in Owerri, Imo State, Nigeria: a cross-sectional study. BMC nursing. 2007. 6(1), 1.

Ngomane S, Mulaudzi FM. Indigenous beliefs and practices that influence the delayed attendance of antenatal clinics by women in the Bohlabelo district in Limpopo, South Africa. Midwifery. 2012. 28(1):30-8.

Nguyễn MH, Gammeltoft T, Rasch V. Situation analysis of quality of abortion care in the main maternity hospital in Hải Phòng, Viet Nam. Reproductive health matters. 2007;15(29):172-82.

Nyango DD, Mutihir JT, Laabes EP, Kigbu JH, \& Buba M. Skilled attendance: the key challenges to progress in achieving MDG-5 in north central Nigeria. African journal of reproductive health. 2014. 14(2), 129-138. 
Obare F, van der Kwaak A, \& Birungi H. Factors associated with unintended pregnancy, poor birth outcomes and post-partum contraceptive use among HIV-positive female adolescents in Kenya. BMC women's health. 2012; 12(1), 34.

Ochako R, Mbondo M, Aloo S, Kaimenyi S, Thompson R, Temmerman M, \& Kays M. Barriers to modern contraceptive methods uptake among young women in Kenya: a qualitative study. BMC Public Health. 2015; 15, 118. doi: 10.1186/s12889-015-1483-1

Oduro-Mensah E, Kwamie A, Antwi E, Bamfo SA, Bainson HM, Marfo B, Coleman MA, Grobbee DE, \& Agyepong IA. Care decision making of frontline providers of maternal and newborn health services in the greater Accra region of Ghana. PloS one. 2013. 8(2), e55610.

Okokon IB, Oku AO, Agan TU, Asibong UE, Essien EJ, \& Monjok E. An Evaluation of the Knowledge and Utilization of the Partogragh in Primary, Secondary, and Tertiary Care Settings in Calabar, South-South Nigeria. International journal of family medicine. 2014.

Opiah MM, Ofi AB, Essien EJ, \& Monjok E. Knowledge and utilization of the partograph among midwives in the Niger Delta Region of Nigeria. African journal of reproductive health. 2012. 125-132.

Palen L, Smith EA, Flisher AJ, Caldwell LL, \& Mpofu E. Substance use and sexual risk behavior among South African eighth grade students. The Journal of Adolescent Health. 2006; 39, 761-763. doi:10.1016/j.jadohealth.2006. 04.016.

Pallant J. SPSS survival manual. McGraw-Hill Education (UK). 2013.

Panday S, Makiwane M, Ranchod C, Letsoala T. Teenage pregnancy in South Africa: with a specific focus on school-going learners. 2009. Pretoria

Patton GC, Sawyer SM, Santelli JS, Ross DA, Afifi R, Allen NB, Arora M, Azzopardi P, Baldwin W, Bonell C, Kakuma R. Our future: a Lancet commission on adolescent health and wellbeing. The Lancet. 2016 Jun 11;387(10036):2423-78. 
Peck C, McCall M, McLaren B, \& Rotem T. Continuing medical education and continuing professional development: international comparisons. Bmj. 2000; 320(7232), 432-435.

Pettifor A E, Rees HV, Kleinschmidt I, Steffenson AE, MacPhail C, Hlongwa-Madikizela L, et al. Young people's sexual health in South Africa: HIV prevalence and sexual behaviors from a nationally representative household survey. Aids. 2005; 19(14), 1525-1534.

Preventing early pregnancy: What the evidence says. Geneva: WHO; 2011

PubChem: Open Chemistry Database.

https://pubchem.ncbi.nlm.nih.gov/compound/methaqualone\#section=Top Viewed on 14 April 2016

Raine TR, Harper CC, Rocca CH, Fischer R, Padian N, Klausner JD, \& Darney PD. Direct access to emergency contraception through pharmacies and effect on unintended pregnancy and STIs: a randomized controlled trial. Jama. 2005. 293(1), 54-62.

Ramkissoon A, Searle C, Burns C, Beksinska M. Sexual and reproductive health and rights: reflections on the Millennium Development Goals. South African Health Review. 2010 Jan 1;2010(1):33-48.

Rankin K, Jarvis-Thiébault J, Pfeifer N, Engelbert M, Perng J, Yoon S and Heard A. Adolescent sexual and reproductive health: an evidence gap map. Evidence Gap Map Report 3. New Delhi: International Initiative for Impact Evaluation (3ie). 2016.

Rankin K, Heard A, Diaz N. Adolescent sexual and reproductive health. Scoping the impact of programming in low-and middle-income countries.

Rehnström Loi U, Gemzell-Danielsson K, Faxelid E, Klingberg-Allvin M. Health care providers' perceptions of and attitudes towards induced abortions in sub-Saharan Africa and Southeast Asia: a systematic literature review of qualitative and quantitative data. BMC public health. 2015;15(1):139. 
Reddy P, James S, Sewpaul R, Yach D, Resnicow K, Sifunda S, Mthembu Z, Mbewu A. A decade of tobacco control: The South African case of politics, health policy, health promotion and behaviour change. SAMJ: South African Medical Journal. 2013; 103(11), 835-840.

Reddy SP, James S, Sewpaul R, Koopman F, Funani NI, Sifunda S. et al. Umthente Uhlaba Usamila- The South African Youth Risk Behaviour Survey 2008. Cape Town: South African Medical Research Council, 2010

Reddy SP, Panday S, Swart D, Jinabhai CC, Amosun SL, James S. et al. Umthenthe uhlaba usamila-The South African youth risk behaviour survey 2002. Cape Town: South African Medical Research Council, 2003.

Reddy P, James S, McCauley A. Programming for HIV prevention in South African schools: a report on program implementation. 2003

Richter HI, Ouattara GG, Heilenkotter I, Gueye D, \& Fischer C. The right skills for health systems development. World Health Forum. 1996; 17(4), 392-394.

Rosen HE, Lynam PF, Carr C, Reis V, Ricca J, Bazant ES, Bartlett LA. Direct observation of respectful maternity care in five countries: a cross-sectional study of health facilities in East and Southern Africa. BMC pregnancy and childbirth. 2015;15(1):1.

Rosenthal JA. Qualitative descriptors of strength of association and effect size. Journal of social service Research. 1996;21 (4):37-59.

Santelli JS, Lindberg LD, Finer LB, \& Singh S. Explaining recent declines in adolescent pregnancy in the United States: the contribution of abstinence and improved contraceptive use. American Journal of Public Health. 2007; 97(1), 150.

Santhya KG, \& Jejeebhoy SJ. Sexual and reproductive health and rights of adolescent girls: Evidence from low and middle-income countries. Global Public Health, 2015. 10(2), 189221. doi:10.1080/17441692.2014.986169 
Schneider H, \& Gilson L. The impact of free maternal health care in South Africa. 1999.

Shisana O, Rehle T, Simbayi LC, Zuma K, Jooste S, Zungu N, et al. South African National HIV Prevalence, Incidence and Behaviour Survey, 2012. Cape Town, HSRC Press. 2014.

Shisana 0, \& Simbayi LC. Nelson Mandela/HSRC study of HIV/AIDS: South African national HIV prevalence, behavioural risks and mass media: household survey 2002. HSRC Press. 2002.

Sidze EM, Lardoux S, Speizer IS, Faye CM, Mutua MM, \& Badji F. Young Women's Access to and Use of Contraceptives: The Role of Providers' Restrictions in Urban Senegal. International perspectives on sexual and reproductive health. 2014. 40(4), 176-183.

Singh S, Bankole A, Woog V. Evaluating the need for sex education in developing countries: sexual behaviour, knowledge of preventing sexually transmitted infections/HIV and unplanned pregnancy. Sex Educ. 2005. 5: 307-31.

Singh S, Darroch JE. Adding it up: costs and benefits of contraceptive service. Estimates for 2012. New York: Guttmacher Institute and UNFPA; 2012http://www. guttmacher.org/pubs/AIU-2012-estimates.pdf.

Silverman JG, Raj A, Mucci LA, \& Hathaway JE. Dating violence against adolescent girls and associated substance use, unhealthy weight control, sexual risk behavior, pregnancy, and suicidality. Jama. 2001; 286(5), 572-579.

Smith KA, Harrison A. Teachers' attitudes towards adolescent sexuality and life skills education in rural South Africa. Sex education. 2013 Jan 1;13(1):68-81.

Somba MJ, Mbonile M, Obure J, \& Mahande MJ. Sexual behaviour, contraceptive knowledge and use among female undergraduates' students of Muhimbili and Dar es Salaam Universities, Tanzania: a cross-sectional study. BMC Women's Health. 2014; 14(94), 8.

Snow R, Laski L, \& Mutumba M.. Sexual and reproductive health: Progress and outstanding needs. Global Public Health, 10(2), 2015; 149-173. 
doi:10.1080/17441692.2014.986178

SPSS I. IBM SPSS statistics version 21. Boston, Mass: International Business Machines Corp. 2012.

Statistics SA. Census 2011 Statistical release: P0301.4. Statistics South Africa. Pretoria: Statistics South Africa. 2012a

Svanemyr J, Amin A, Robles OJ, Greene ME. Creating an enabling environment for adolescent sexual and reproductive health: a framework and promising approaches. Journal of Adolescent Health. 2015 Jan 31;56(1):S7-14.

Taylor M, Dlamini SB, Kagoro H, Jinabhai CC, \& de Vries H. Understanding high school students' risk behaviors to help reduce the HIV/AIDS epidemic in KwaZulu-Natal, South Africa. The Journal of School Health. 2003; 73, 97-100.

Tesch R. (1990). Qualitative research: Analysis types and software tools. Bristol, PA: Falmer.

Tilahun M, Mengistie B, Egata G, \& Reda AA. Health workers' attitudes toward sexual and reproductive health services for unmarried adolescents in Ethiopia. Reprod Health. 2012. 9(1), 19.

Tita ATN, Selwyn BJ, Waller DK, Kapadia AS, \& Dongmo S. Factors associated with the awareness and practice of evidence-based obstetric care in an African setting. BJOG: An International Journal of Obstetrics \& Gynaecology. 2006; 113(9), 1060-1066.

Tlebere P, Jackson D, Loveday M, Matizirofa L, Mbombo N, Doherty T, Wigton A, Treger L, \& Chopra M. Community-Based Situation Analysis of Maternal and Neonatal Care in South Africa to Explore Factors that Impact Utilization of Maternal Health Services. Journal of midwifery \& women's health. 2007;52(4):342-50.

Traoré M, Arsenault C, Schoemaker-Marcotte C, Coulibaly A, Huchon C, Dumont A, \& Fournier P. Obstetric competence among primary healthcare workers in Mali. 
International Journal of Gynecology \& Obstetrics. 2014. 126(1), 50-55.

Troped PJ, Wiecha JL, Fragala MS, Matthews CE, Finkelstein DM, Kim J, \& Peterson KE. Reliability and validity of YRBS physical activity items among middle school students. Medicine and science in sports and exercise. 2007; 39(3), 416-425.

Tsawe M, Susuman AS. Determinants of access to and use of maternal health care services in the Eastern Cape, South Africa: a quantitative and qualitative investigation. BMC research notes. 2014. 7(1): 723. DOI: 10.1186/1756-0500-7-723

Turner KL, Hyman AG, Gabriel MC. Clarifying values and transforming attitudes to improve access to second trimester abortion. Reproductive health matters. 2008. 16(31): 108-16.

Ueno E, Adegoke AA, Masenga G, Fimbo J, \& Msuya SE. Skilled birth attendants in Tanzania: a descriptive study of cadres and emergency obstetric care signal functions performed. Maternal and child health journal. 2015; 19(1), 155-169.

United Nations Population Fund. Adolescent Sexual and Reproductive Health. 2014. http://www.unfpa.org/resources/adolescent-sexual-and-reproductive-health. Accessed 18 November 2016.

United Nations Population Fund. Motherhood in Childhood: Facing the challenge of adolescent pregnancy. 2013. New York: UNFPA.

Varga CA. How gender roles influence sexual and reproductive health among South African adolescents. Studies in family planning. 2003; 34(3), 160-172.

Vivio D, Fullerton JT, Forman R, Mbewe RK, Musumali M, \& Chewe, PM. Integration of the practice of active management of the third stage of labor within training and service implementation programming in Zambia. Journal of Midwifery \& Women's Health. 2010. 55(5), 447-454. 
Vundule C, Maforah F, Jewkes R, \& Jordaan E. Risk factors for teenage pregnancy among sexually active black adolescents in Cape Town. S Afr Med J. 2001; 91(1), 73-80.

Wakgari N, Amano A, Berta M, \& Tessema GA. Partograph utilization and associated factors among obstetric care providers in North Shoa Zone, Central Ethiopia: a cross sectional study. African health sciences. 2015. 15(2), 552-559.

Waldman L, Stevens M. Sexual and reproductive health and rights and mHealth in policy and practice in South Africa. Reproductive health matters. 2015 May 31;23(45):93-102.

Warenius LU, Faxelid E A, Chishimba PN, Musandu JO, Ong'any AA, \& Nissen EB. Nursemidwives' attitudes towards adolescent sexual and reproductive health needs in Kenya and Zambia. Reproductive Health Matters. 2006. 14(27), 119-128.

Watts NC, Mimmie C, Liamputtong P, \& Carolan M. Contraception knowledge and attitudes: truths and myths among African Australian teenage mothers in Greater Melbourne, Australia. J Clin Nurs. 2014; 23(15-16), 2131-2141.

Wheeler SB, Zullig LL, Reeve BB, Buga GA, Morroni C. Attitudes and intentions regarding abortion provision among medical school students in South Africa. International perspectives on sexual and reproductive health. 2012 Sep 1:154-63.

Wilkinson D, Gouws E, Sach M, \& Karim SSA. Effect of removing user fees on attendance for curative and preventive primary health care services in rural South Africa. Bulletin of the World Health Organization. 2001; 79(7), 665-671.

Wood K, \& Jewkes R. Blood blockages and scolding nurses: barriers to adolescent contraceptive use in South Africa. Reproductive Health Matters. 2006; 14(27), 109-118.

Worku AG, Yalew AW, \& Afework MF. Availability and components of maternity services according to providers and users perspectives in North Gondar, northwest Ethiopia. Reproductive health. 2013. 10(1), 43.

World bank Data. Maternal mortality ratio (modeled estimate, per 100,000 live births). 
World Health Organization. Adolescent pregnancy. Fact sheet No. 364.September 2014. http://www.who.int/mediacentre/factsheets/fs364/en/. Accessed May 19th, 2016.

World Health Organization. UN Sustainable Development Summit 2015. http://www.who.int/mediacentre/events/meetings/2015/un-sustainable-developmentsummit/en/. Accessed on 09 February 2016.

World Health Organization (WHO). Maternal, newborn, child and adolescent health. http://www.who.int/maternal child adolescent/topics/maternal/adolescent_pregnancy /en/. Accessed 14 April 2016.

Yeji F, Shibanuma A, Oduro A, Debpuur C, Kikuchi K, Owusu-Agei S, Gyapong M, Okawa S, Ansah E, Asare GQ, Nanishi K. Continuum of care in a maternal, newborn and child health program in Ghana: Low completion rate and multiple obstacle factors. PloS one. 2015. 10(12):e0142849.

Yisma E, Dessalegn B, Astatkie A, \& Fesseha, N. Knowledge and utilization of partograph among obstetric care givers in public health institutions of Addis Ababa, Ethiopia. BMC pregnancy and childbirth. 2013. 13(1), 1.

Zielinski Gutierrez E, Magnani R, Lipovsek V, Belmonte LR. Who can we trust with our problems. Barriers to Adolescent Use of Reproductive Health Services in. 2001; 3. 


\section{Summary}

The studies reported in this dissertation provide more insight into the behaviours of healthcare workers affecting adequate provision of sexual and reproductive healthcare (SRH) services to adolescents in South Africa (SA). Insights into the behaviours of healthcare workers are important in identifying areas needing improvement, so as to enhance quality of SRH services and ultimately improve access to and utilization of SRH services by young people at large.

Globally, access to and utilization of SRH services by adolescents is poor. Hence, the WHO, UNPFA, UNICEF, and the GSWCAH of 2016-2030 have highlighted the need to maximize the efforts to increase adolescents' access to and utilization of SRH services in order to improve their health outcomes.

Healthcare workers' behaviours towards adolescents seeking SRH services are crucial in realizing these global efforts. However, if behaviours of healthcare workers are negative towards adolescents seeking SRH services, access to and utilization of SRH services by adolescents is likely to be negatively affected. Thus, the ultimate goal of the studies reported in this dissertation was to identify the negative behaviours of healthcare workers in order to inform future development of specific interventions to change the behaviours and subsequently improve provision of quality SRH services as well as access to and utilization of SRH services by adolescents in SA.

Chapter 2 reports an epidemiological overview of adolescents' needs for SRH services from three national surveys (YRBS), representative of a total of 31816 school going adolescents in SA. The overall prevalence of having ever been pregnant among the combined 3-survey sample was self-reported to be $11.0 \%$. The odds for ever been pregnant were higher for girls who had 2 or more sexual partners (OR: 1.250, 95\% CI: 1.039 - 1.503), girls who ever used alcohol before sex (OR: 1.373, 95\% CI: 1.004 - 1.878), and girls who used mandrax (OR: 1.968, 95\% CI: 1,243 - 3.117). Girls continue to become pregnant at unacceptably high rates in SA therefore, there is a need to address adolescents' sexual and reproductive health needs, and several health risk behaviours, including substance use, that are associated with teenage pregnancy in SA. 
Chapter 3 describes healthcare workers' behaviours that affect the utilization of SRH services, and quality of care they provide in sub-Saharan Africa (SSA). A systematic review of literature was conducted to identify studies focusing on healthcare workers' behaviors and personal determinants associated with providing adequate SRH services in SSA (January 1990 - October 2015). A total of 35 studies met all the inclusion criteria. Negative behaviours and attitudes of healthcare workers, as well as other personal determinants, such as poor knowledge and skills of SRH services, and related factors, like availability of essential drugs and equipment are associated with provision of inadequate SRH services. Healthcare workers' negative behaviours and attitudes can discourage women in general to access and utilize SRH services, and especially young women. Knowledge of SRH services, including basic emergency obstetric care (EmOC) is insufficient among healthcare workers in SSA.

Chapter 4 describes the predictors of intentions to provide SRH services to adolescents in SA. A total of 190 healthcare workers completed a cross-sectional survey on knowledge of maternal and child healthcare (MCH) and family planning (FP) services, attitude towards family planning services, subjective norms regarding $\mathrm{MCH}$ and FP services, self-efficacy with MCH and FP services, and intentions to provide MCH and FP services to adolescents. Self-efficacy to conduct MCH services $(\beta=.55, \mathrm{p}<0.01)$ and years of experience as a nurse/midwife $(\beta=.14, \mathrm{p}<0.05)$ were associated with stronger intentions to provide the services. Self-efficacy to provide FP services $(\beta=.30, p<0.01)$ was associated with stronger intentions to provide FP services. Self-efficacy has a strong and positive association with the intentions to provide both MCH and FP services. There is a need to improve and strengthen nurses' and midwives' self-efficacy in conducting both MCH and FP services in order to improve the quality and utilization of the services by adolescents in South Africa.

Chapter 5 describes the beliefs, behaviours and motivations of healthcare workers affecting adequate provision of SRH to adolescents in Cape Town, SA. Twenty-four healthcare workers in public SRH services participated in this qualitative study through focus group discussions (FGDs). SRH nurses indicated that they are still experiencing challenges with termination of pregnancy (TOP) due to their opposing beliefs and values. Some nurses felt that they had insufficient SRH skills, which hinder their provision of adequate SRH services to adolescents. Healthcare workers are faced with numerous 
challenges when providing SRH services to adolescents. Providing SRH training and refresher courses to improve the nurses' skills in SRH services are required.

Chapter 6 describes healthcare workers' perceptions of adolescents seeking and or utilizing SRH services in Cape Town. Twenty-four healthcare workers in public SRH services participated in this qualitative study through FGDs. SRH nurses are generally supportive of adolescents who ask for and use contraceptives. The nurses perceived noncompliance to FP regimens and repeated requests of TOPs as irresponsible behaviours; which particularly frustrated them. The subsequent nurse-adolescent interactions compromised by these perceived irresponsible behaviours of adolescents sometimes appeared to hinder access to and utilization of SRH services by adolescents. A good adolescent-nurse relationship may help prevent adolescents' risky behaviours, and facilitate adolescents' access to and utilization of SRH services. Nurses' counseling and communication skills should be improved in order to effectively interact with their adolescent clients, and help improve adolescent' access to and utilization of SRH services.

Chapter 7 summarizes the main findings from the different studies described above, as well as discusses methodological considerations and implications for future research and practical applications. From the main findings of these studies, it can be concluded that healthcare workers have negative behaviours towards providing SRH services, especially TOP services. Furthermore, the findings of these studies show that healthcare workers in SRH services are battling with their own personal views and beliefs with regards to some SRH services that they have to provide to adolescents. The findings of these studies provide more insights into underlying reasons for healthcare workers negative behaviours towards adolescents seeking SRH services. Additionally, it can also be concluded that healthcare workers' behaviours and personal determinants are influenced by a number of other factors inside and outside of the healthcare systems and all these factors need to be taken into consideration when developing interventions which aim to improve adolescents' access to and utilization of SRH services. Healthcare workers' negative behaviours and attitudes towards adolescents seeking SRH services have potential to act as barrier to services and therefore should be addressed. 


\section{Valorization}

The findings of this dissertation provide an overview of healthcare workers' behaviours and personal determinants, and their associated factors affecting adequate provision of sexual and reproductive healthcare (SRH) services to adolescents in South Africa (SA). The department of health has adopted some of the recommendations in this dissertation. For example, the school outreach programs, as well as the adaptions in the existing youth clinics currently being implemented in some communities are in line with the strong recommendations from this dissertation, in order to improve adolescents' access to and utilization of SRH services in the country.

Chapter 2 emphasizes the importance of regular monitoring of teenage pregnancy and associated factors in order to determine areas where the department of health can place maximum efforts in designing and implementing programs to help lower the teenage pregnancy rates. Adolescent sexual and reproductive health behaviours, such as contraceptive use and safe sex practices are important health issues in adolescent health and wellbeing, globally. Yet, access to and utilization of SRH services is poor, especially in SA (Wood et al., 2006; Biddlecom et al., 2007). Thus, efforts to improve adolescents' access to and utilization of SRH services from the healthcare systems of SA need to be intensified. Both the department of health and the department of education need to work together in optimizing the SRH services for adolescents in SA. This collaboration is already implemented in some aspects of the public healthcare systems, and has shown some benefits for the communities served. For example, the family planning outreach programs being implemented in some schools in the country have been reported to aid in the reduction of teenage pregnancies and abortions in the areas covered, and improved adolescents' access to and utilization of SRH services. Improved access to and utilization of SRH services by adolescents, especially the use of contraceptives is necessary to improve their sexual and reproductive health outcomes, reduce teenage pregnancy and improve adolescents' health and wellbeing.

The findings in this dissertation provide information on the factors contributing to the poor access to and utilization of SRH services by adolescents, and thereby highlight the specific areas of intervention for improvement. For example, the findings reported in 
Chapter 3 point to the limited knowledge and skills of healthcare workers as the underlying factors associated with inadequate quality of SRH services. This dissertation recommends the mandatory inclusion and enforcement of continuous education and trainings of healthcare workers in SRH services within the healthcare systems program as means to address the limited SRH knowledge and skills. Moreover, the findings in Chapters 5 and 6 point to the societal norms and religious beliefs of healthcare workers as the underlying factors associated with their negative behaviours and attitudes towards providing SRH services to adolescents. Engaging community leaders and members in discussions on adolescent sexuality and benefits of family planning services for adolescents, as well as reiterating the professional roles and responsibilities of healthcare workers are amongst the recommendations for addressing the negative societal norms identified by the research reported in this dissertation.

Healthcare workers' negative beliefs, attitudes, lack of knowledge and skills and low selfefficacy in providing SRH services to adolescents are the main determinants of the negative behaviours of healthcare workers, as reported in Chapters 3-6. With regards to the beliefs and attitudes, this dissertation calls for community engagement in order to stimulate support for contraceptive use among young people. Healthcare workers are human beings living in a society that has specific social norms and moral values, thus addressing healthcare workers' beliefs and attitudes that stand in the way of adequate quality SRH service provision to adolescents requires a multilevel approach. The multilevel approach should involve the community members, such as parents, the youth, and the healthcare workers, whereby the benefits of SRH services provision, the beliefs and attitudes related to adolescent sexuality and contraceptive use, and the healthcare professionals' value clarification are discussed.

The foundation for professional development (FPD) in South Africa currently offers continuous professional development (CPD) trainings and refresher courses, which include some of the SRH related trainings and workshops; however, they are not mandatory. Thus, some healthcare workers may not attend these training courses, as they deem them unnecessary and remain behind with new knowledge and information related to SRH services, skills and practices. Hence, this dissertation makes strong recommendation to the department of health and the department of education to highly prioritize continuous SRH education and training of healthcare workers and should be 
mandatory to all SRH healthcare workers in order to improve their SRH knowledge and skills. Given the long-term consequences and serious health implications of poor reproductive health outcomes, serious financial investments into the SRH program are needed. Similar to the prioritization and management of HIV/AIDS that was escalated during the peak of the epidemic, the healthcare systems can apply the same strategy in prioritizing SRH services. This needs to be a concerted effort from both government departments, the department of education and the department of health, and a scaling up of human resources in the healthcare systems is necessary to attain the prioritization of SRH services, including the continuous education and training recommendations. Without the necessary human resources, mandatory continuous SRH trainings programs will not be implemented successfully, leaving healthcare workers ill-equipped with recent and upto-date SRH information.

Poor SRH knowledge and skills is associated with poor self-efficacy and compromises quality of care. The poor quality of care has a potential to deter clients from accessing the SRH services. Therefore, it is important that the healthcare systems invest efforts to improve healthcare workers' SRH knowledge and skills in order to improve the quality of care in SRH services, and ultimately enhance access to utilization of these services. Hence, this dissertation recommends that continuous SRH education and trainings of healthcare workers be incorporated and enforced into the healthcare systems policies for professional development. The importance of comprehensive knowledge and skills to adequately provide SRH services to adolescents cannot be over-emphasized and should be prioritized, as it is critical in improving their access to and utilization of SRH services.

Healthcare workers in public primary healthcare services in SA, particularly nurses, are very critical personnel in the provision of SRH services to adolescents. Thus, healthcare workers' behaviours, personal values, and morale play a significant role in adolescents' access to and utilization of SRH services. Healthcare workers' negative behaviours and attitudes can significantly hinder the provision of adequate quality SRH services to young people, especially to adolescent girls. For adolescents to fully access and utilize SRH services, they need to be served by welcoming and youth-friendly healthcare workers who have a positive attitude towards adolescents seeking SRH services. This recommendation has been partially implemented in the public healthcare systems. However, as reported in Chapters 5 and 6, the youth clinics and SRH services are not 
prioritized in some clinics. This dissertation therefore, advocates for the prioritization of adolescents' SRH services within the healthcare systems of SA, at large.

A dedicated healthcare worker for adolescent SRH services, who only attends to adolescents and does not get pulled into other health facility emergencies, could be one way of prioritizing adolescent SRH services. Secondly, a revision of the current health facility working hours to accommodate school-going adolescents could be another way of prioritizing adolescent SRH services. For example, health facilities should be open until after school hours to maximize adolescents' access to SRH services. This can be achieved by allowing the dedicated adolescent healthcare worker to resume his/her working hours a little later than the other healthcare workers. For example, he/she starts work at $11 \mathrm{~h} 00$ until 19 h00 to complete her 8-hour daily working hours.

The National Adolescent Sexual and Reproductive Health and Rights (ASRH\&R) Framework Strategy is a starting point for the prioritization of adolescents' SRH services in the country. However, its full implementation cannot be taken for granted. Continuous monitoring and evaluation measures of the strategy's implementation and other SRH guidelines need to be put in place and used to decide on future efforts. Health facility managers and other key personnel in the healthcare systems need to be advocates of the strategy on adolescent SRH services, thereby giving adolescents who are seeking SRH services priority. 


\section{Acknowledgements}

First and foremost, I would like to thank God for His abundant blessings and guidance.

My heartfelt gratitude goes to the European Commission (through EUROSA), the Dutch Ministry for Foreign Affairs (through Nuffic) for the financial support provided for this PhD project.

The financial support of the National Research Foundation (NRF) towards this research is also hereby acknowledged. Opinion expressed and conclusions arrived at, are those of the authors and are not necessarily to be attributed to the NRF.

To my diligent supervisors, Prof Bart van den Borne, Prof Priscilla Reddy, and Dr Rik Crutzen, thank you for your academic, personal, and moral support throughout this $\mathrm{PhD}$ trajectory.

My heartfelt, special thanks to Dr Rik Crutzen for his continuous dedication and encouragement, for going over and beyond to keep me motivated and inspired from start to finish of this research, words are just not enough to express my gratitude to you. I am eternally indebted to you!

My sincere gratitude is extended to the collaboration team, Prof Nicolette Roman, Dr Anja Krumeich and Ms Amanda Hlabeni for their intellectual and technical support provided in this research.

To Ms Lori Mees, the mother I never knew I had or needed, thank you so much for going the extra mile for me and continue to resolve my crises to the best of your ability. Your role throughout this $\mathrm{PhD}$ trajectory will forever be treasured, thank you!

I thank my colleagues from Maastricht University; Leon Kolenburg who has played a role of more than a big brother to me, Patricia von Schenckendorff, Kim van Hensberg, Steffi Gomez, Latifa Abidi, Francine Schneider, Aziza Mukhayer, Hans Dupont, Roy Andrew, TMO team, who made my stay in Maastricht at the Health Promotion Department more than enjoyable. Thank you all for being there when I needed you and for all the support 
you offered me.

To my friends; Rachel Brown, Martha Shiell aka eMS, Chantal Ingabire, Steffi Gomez, Stefan Gruijters, Nikky Adams-Quackenbush, Birgit Veskioja, and Ben Schultz, thank you for making my "home-away-from-home" time the best time ever.

A special thanks to my housemates; Roel Brouwers and Antonin Allard for making me feel at home in the apartments we shared, and for your constant support.

To the healthcare workers who participated enthusiastically in this research, without your contribution this dissertation would not have been possible. For that, I thank you from the bottom of my heart.

To my specialist doctor, Dr. Astrid Oude Lashof from azM who took great care of my health-thank you Dr!

A special thanks to Ioanna Saliari and her family for making sure I survive the European cold winter days.

To the Honorable Minister of Science and Technology, Min. Naledi Pandor and to Dr Phethiwe Matutu, thank you for your support.

My sincere gratitude goes to my sister; Akhona Jonas, and to my family in its entirety, thank you for your support throughout this journey.

Last but not least, to my dearest husband; Themba Sithole, thank you for your overflowing love and support throughout this journey. Specifically, thank you for supporting my dream and for putting up with the ups and downs of being apart for this long.

This dissertation is dedication to my late father, Michael Jonas who passed away while I was in the middle of the PhD trajectory. I wish you would have been here to see me on this day. Baba, ngiyazi ukuthi uyaziqhenya ngami lapho ukhona. Lala ngokuthula Mbathane omuhle. [Daddy, I know you are proud of me wherever you are. Rest in peace]. 


\section{Author resume}

Ncebakazi Kim Jonas was born in Butterworth, in the Eastern Cape province of South Africa. She completed a Bachelor of Complementary Health Sciences and subsequently a Bachelor of Complementary Medicine at the University of Western Cape (UWC). She also obtained a master's degree from the same university in Child and Family Studies, focusing on quality of life enjoyment and satisfaction (Q-LES) among individuals living with HIV and AIDS in 2013. Her professional career started at the Medical Research Council (MRC) of SA as a clinical research intern, after completing her clinical practice training at Tygerberg Hospital in 2010. At the MRC she was conducting cytotoxic studies related to medicinal plant-based drugs for diabetes type II and malaria in preparation for clinical trials to test for drug efficacy. At this stage, Kim realized that "hard" science is not necessarily her passion. She then left to the Human Sciences Research Council (HSRC), where she started as a junior researcher in late 2011. She worked on various national flagship projects including the South African National HIV Prevalence, Incidence, Treatment, Behaviour and Communication Survey, 2012 (SABSSM) IV, the South African National Health And Nutrition Examination Survey, 2011/2012 (SANHANES), HIV prevalence and risk behaviours among men who have sex with men (MSM) in Cape Town, Durban and Johannesburg, South Africa (MSM project), and the Improvement of Maternal and Child Morbidity and Mortality Surveillance System (MIMMS) of South Africa project as a provincial coordinator and data analyst. Her main responsibilities included overseeing fieldwork and data collection activities, data management and analysis, as well as producing cumulative progress reports for the projects, and assisted in report writing of the projects. In 2013, as a researcher now, she was made a project director for the HIV and Substance Use Prevention for South African Youth and Families (SASTYLE) project where she was responsible to oversee all project related activities, including data management and analysis. From these projects, her desire to pursue doctoral studies and become a highly qualified researcher was initiated. In late 2014, while she was seeking funding and universities to pursue her PhD studies, she was awarded a EUROSA fellowship to conduct research related activities towards her PhD project at Maastricht University in the Netherlands for 18 months. EUROSA is a partnership between SA and the European commission for capacity development. She also received a Nuffic grant for four years to conduct her $\mathrm{PhD}$ research beginning of 2015. Her PhD trajectory fully resumed at the beginning of 2015 focusing on uncovering healthcare workers behaviours 
and personal determinants associated with adequate provision of sexual and reproductive healthcare services to adolescents in SA. Early 2017, Kim was offered a short-term internship at the World Health Organization (WHO) headquarters in Geneva, in the reproductive health program. At the WHO, she was involved in conducting reviews in the field of adolescent sexual and reproductive healthcare (SRH) services, the performance of healthcare workers in SRH services, as well as developing a combination guideline tool for all adolescent related health guidelines. She has acquired extensive international experience in her field of interest, which is adolescent sexual and reproductive health and wellbeing. Combining her international experience and previous professional experience, she is very resourceful to her country, SA and to the international public health field. She has started a postdoctoral fellowship at the University of Cape Town (UCT) in SA, where she hopes to pursue research focusing on improving adolescent contraceptive use in order to halt the teenage pregnancy rates in the country. 


\section{Publications}

Jonas K, Crutzen R, van den Borne B, Reddy P. Healthcare workers' behaviors and personal determinants associated with providing adequate sexual and reproductive healthcare services in sub-Saharan Africa: a systematic review. BMC Pregnancy and Childbirth. 2017 Mar 13; 17(1):86.

Jonas K, Reddy P, van den Borne B, Sewpaul R, Nyembezi A, Naidoo P, Crutzen R. Predictors of nurses' and midwives' intentions to provide maternal and child healthcare services to adolescents in South Africa. BMC Health Services Research. 2016 Nov $15 ; 16(1): 658$.

Jonas K, Crutzen R, van den Borne B, Sewpaul R, Reddy P. Teenage pregnancy rates and associations with other health risk behaviours: a three-wave cross-sectional study among South African school-going adolescents. Reproductive health. 2016 May 4;13(1):1.

Reddy P, Sewpaul R, and Jonas K. Teenage Pregnancy In South Africa. HSRC Press. Policy Brief. 2016.

Jonas K, Crutzen K, van den Borne B, Reddy P. Healthcare workers' behavior and personal determinants associated with providing sexual and reproductive healthcare services to teenagers in sub-Saharan Africa: a systematic review protocol. PROSPERO 2015: CRD42015017509 Available from

http://www.crd.york.ac.uk/PROSPERO/display_record.asp?ID=CRD42015017509

Reddy P, Zuma K, Shisana 0, Jonas K, Sewpaul R. Adult Tobacco Use Prevalence in South Africa: Results from the First South African National Health and Nutrition Examination Survey. South African Medical Journal. 2015; 105 (8): 648-655.

Jonas K, Naidoo P, Roman N, Saul LA, and Feixa G. Predictors of quality of life enjoyment and satisfaction (Q-LES) among HIV positive individuals in South Africa (SA). Journal of Psychology in Africa. 2015, 25(2), 95-99. 
Naidoo P, Donenberg G, Davids A, Jonas K, Vermaak R, Simbayi L, Kagee A, Ward C, and Emersen E. Exploring risk and protective mechanisms associated with HIV infection among adolescents in South Africa. Journal of Psychology in Africa. 2014; 24: 3, 232-240. 